\title{
Holonomic Quantum Fields. V
}

\author{
By \\ Mikio SAto*, Tetsuji Miwa*† and Michio Jimbo*
}

\section{Introduction}

The present chapter $\mathrm{V}$ of our series is an application of the theory of rotation ([1]) to the lattice models. Included here are the two-dimensional Ising model ([5], [6]), a bosonic counterpart of it, the one-dimensional XY model ([12]) and the free fermion model ([14] [15] [16]). In each case we shall compute exactly the norm representation of spin operators, and hence their $n$-point correlation functions. The materials in this article are "time-ordered" according to the development, but we could have unified the treatment by using the path integral formalism exposed in Section 5.4 (symplectic case) and in Section 5.7 (orthogonal case). Since the first announcement of our result on the Ising model ([2] [4]), there have appeared several independent papers [9] [10] [11] that deal with the exact computation of $n$-point functions. We emphasize that these results are made most transparent by considering directly the explicit form of spin operators. (For instance the arbitrariness in the infinite series expression of $n$-point functions for $T>T_{c}$ is neatly described in this way. See p. 548.)

The plan of this paper is as follows. The first three sections 5.1-5.3 are devoted to the Ising model. We shall see that a systematic application of the original method of Onsager ([5]) enables one to express explicitly not only the free energy but also the spin operator itself. We first review the diagonalization procedure of the Hamiltonian (see [5] [7]) in Section 5.1, and compute the norm representation of spin operators in Section 5.2. Using these results we derive in Section 5.3 infinite series expressions for $n$-point correlation functions (an application of the product formula in [1]). We also verify their convergence and several symmetry properties. In Section 5.4 we present a two-

Received December 9, 1978.

* Research Institute for Mathematical Sciences, Kyoto University.

$\dagger$ Partially supported by the Sakkokai foundation. 
dimensional lattice model which constitutes the symplectic version of the Ising model. The reader will easily see that the path integral formulation utilized here admits of an immediate extension to give higher dimensional similar models. In the next Section 5.5 we take the scaling limit, and show that the spin operators in Sections 5.2 and 5.4 are scaled to give $\varphi_{F}(x), \varphi^{F}(x)$ and $\varphi_{B}(x)$ introduced in [2] [3]. In Section 5.6 we treat the 1-dimensional $X Y$ model (cf. [13]). This time the spin operators give in the various scaling regions $\varphi_{F}(x), \varphi^{F}(x)$, their time-derivatives, and also the tensor products of their copies. Lastly in Section 5.7 we consider the free fermion model, to which we refer as the orthogonal model in contrast with the one in Section 5.4. Our path integral treatment differs from the methods in the literature [14] [15] [16] and seems to be a simpler one. This model includes as a special case various Ising-like models, such as those for the triangular (cf. [18]) or the generalized square lattice, so that the n-point functions for these models are obtained exactly. (For the latter we may deal with only the vertices of the same type.)

The authors would like to thank Professor M. Suzuki for informing them of related literatures.

\section{Chapter V. Spin Operators in Various Lattice Models}

\section{§5.1. Diagonalization of the Hamiltonian}

We shall review here the diagonalization procedure of the Hamiltonian of the 2-dimensional rectangular Ising lattice. The content of this section is well known (see [5], [7]), but we have included it here so as to make this paper accessible to non-specialists, and also to fix the notations.

We consider a rectangular lattice of size $M \times N$, where a spin variable $\sigma_{m n}= \pm 1$ is attached to each site $(m, n)(0 \leqq m \leqq M-1,0 \leqq n \leqq N-1)$. The total energy of this system is given by

$$
E(\sigma)=-E_{1} \sum_{m=0}^{M-1} \sum_{n=0}^{N-1} \sigma_{m n} \sigma_{m+1 n}-E_{2} \sum_{m=0}^{M-1} \sum_{n=0}^{N-1} \sigma_{m n} \sigma_{m n+1}
$$

where $E_{1}, E_{2}>0$ are interaction strengths. We have chosen the cyclic convention $\sigma_{m+M k, n+N l}=\sigma_{m n}, k, l \in \boldsymbol{Z}$ (i.e. the lattice is wrapped on a torus). Our main objectives are the grand partition function 


$$
Z_{M N}=\sum_{(\sigma)} e^{-\beta E(\sigma)}
$$

and the correlation functions for arbitrary lattice points $\left(m_{j}, n_{j}\right)(j=1, \ldots, k)$ :

$$
\rho_{k}\left(\left(m_{1}, n_{1}\right), \ldots,\left(m_{k}, n_{k}\right)\right)=Z_{M N}^{-1} \sum_{(\sigma)} \sigma_{m_{1} n_{1}} \cdots \sigma_{m_{k} n_{k}} e^{-\beta E(\sigma)} .
$$

In (5.1.2) and (5.1.3) the sum is taken over $2^{M N}$ possible spin configurations $\sigma_{00}= \pm 1, \ldots, \sigma_{M-1, N-1}= \pm 1$, and $\beta=1 / k T>0$ ( $k$ : the Boltzmann constant $T$ : temperature).

We shall follow the method of transfer matrix. For an $M$-vector $\boldsymbol{\sigma}=$ $\left(\sigma_{0}, \sigma_{1}, \ldots, \sigma_{M-1}\right)$ with entries $\sigma_{i}= \pm 1$, we set

$$
e_{\sigma}=e_{\sigma_{0}} \otimes \cdots \otimes e_{\sigma_{M-1}}, \quad e_{+1}=\left(\begin{array}{l}
1 \\
0
\end{array}\right), \quad e_{-1}=\left(\begin{array}{l}
0 \\
1
\end{array}\right) .
$$

These $2^{M}$ vectors $\left\{e_{\sigma}\right\}$ constitute a basis of $\left(\boldsymbol{C}^{2}\right)^{\otimes M}$. We introduce matrices $V_{1}$, $V_{2}$ whose $\left(e_{\sigma}, e_{\sigma^{\prime}}\right)$-elements are given by

$$
\begin{aligned}
& \left(V_{1}\right)_{\sigma \sigma^{\prime}}=\delta_{\sigma \sigma^{\prime}} \exp \left(K_{1} \sum_{m=0}^{M-1} \sigma_{m} \sigma_{m+1}\right) \\
& \left(V_{2}\right)_{\sigma \sigma^{\prime}}=\exp \left(K_{2} \sum_{m=0}^{M-1} \sigma_{m} \sigma_{m}^{\prime}\right)
\end{aligned}
$$

where $\delta_{\sigma \sigma^{\prime}}=\delta_{\sigma_{0} \sigma_{0}^{\prime}} \cdots \delta_{\sigma_{M-1} \sigma_{M-1}^{\prime}}$ and

$$
K_{1}=\beta E_{1}, \quad K_{2}=\beta E_{2} .
$$

The definition (5.1.2) then reads

$$
\begin{aligned}
Z_{M N} & =\sum_{\sigma_{0}} \cdots \sum_{\sigma_{N-1}}\left(V_{1}\right)_{\sigma_{0} \sigma_{0}}\left(V_{2}\right)_{\sigma_{0} \sigma_{1}} \cdots\left(V_{1}\right)_{\sigma_{N-1} \sigma_{N-1}}\left(V_{2}\right)_{\sigma_{N-1} \sigma_{0}} \\
& =\operatorname{trace}\left(V_{1} V_{2}\right)^{N}
\end{aligned}
$$

where $\sigma_{n}=\left(\sigma_{0 n}, \ldots, \sigma_{M-1 n}\right)(n=0,1, \ldots, N-1)$. If we set

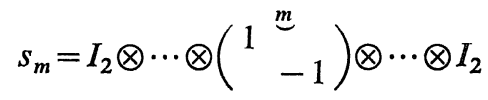

$$
\begin{aligned}
& (m=0,1, \ldots, M-1) \\
& C_{m}=I_{2} \otimes \cdots \otimes\left(1^{\stackrel{m}{ }} 1\right) \otimes \cdots \otimes I_{2}
\end{aligned}
$$

$V_{1}, V_{2}$ are written as

$$
\begin{aligned}
& V_{1}=\exp \left(K_{1}\left(s_{0} s_{1}+s_{1} s_{2}+\cdots+s_{M-1} s_{0}\right)\right) \\
& V_{2}=\left(2 \sinh 2 K_{2}\right)^{M / 2} \exp \left(K_{2}^{*}\left(C_{0}+C_{1}+\cdots+C_{M-1}\right)\right) .
\end{aligned}
$$

Here for $K>0, K^{*}=K^{*}(K)>0$ is determined by the formula 


$$
\begin{aligned}
& \left(\begin{array}{ll}
e^{K} & e^{-K} \\
e^{-K} & e^{K}
\end{array}\right)=(2 \sinh 2 K)^{1 / 2} e^{K^{*}\left({ }_{1}{ }^{1}\right)} \\
& \tanh K^{*}=e^{-2 K}
\end{aligned}
$$

The operators $\left\{s_{m}, C_{m}\right\}$ satisfy the following relations.

$$
\begin{aligned}
& s_{m^{\prime}} s_{m^{\prime}}=s_{m^{\prime}} s_{m}, \quad s_{m}^{2}=1 \\
& C_{m} C_{m^{\prime}}=C_{m^{\prime}} C_{m}, \quad C_{m}^{2}=1 \\
& s_{m} C_{m^{\prime}}=C_{m^{\prime}} s_{m}\left(m \neq m^{\prime}\right), \quad s_{m} C_{m}=-C_{m^{\prime}} s_{m} \\
& \quad\left(m, m^{\prime}=0,1, \ldots, M-1\right) .
\end{aligned}
$$

Making use of the symmetrized transfer matrix

$$
V=V_{1}^{1 / 2} V_{2} V_{1}^{1 / 2}=V_{1}^{-1 / 2}\left(V_{1} V_{2}\right) V_{1}^{1 / 2}
$$

the partition function is given by

$$
Z_{M N}=\operatorname{trace} V^{N} \text {. }
$$

Similar reasoning yields the following expression for correlation functions. Taking into account the symmetry of $\rho_{k}$ in its arguments we may assume $n_{1}$ $\leqq \cdots \leqq n_{k}$.

$$
\begin{aligned}
& \rho_{k}\left(\left(m_{1}, n_{1}\right), \ldots,\left(m_{k}, n_{k}\right)\right) \\
= & \frac{\operatorname{trace}\left(s_{m_{1} n_{1}} \cdots s_{m_{k} n_{k}} V^{N}\right)}{\operatorname{trace} V^{N}} \quad\left(n_{1} \leqq \cdots \leqq n_{k}\right)
\end{aligned}
$$

where

$$
\begin{aligned}
s_{m n}= & V_{1}^{-1 / 2}\left(V_{1} V_{2}\right)^{n} s_{m}\left(V_{1} V_{2}\right)^{-n} V_{1}^{1 / 2} \\
= & V^{n} s_{m} V^{-n} \\
& (0 \leqq m \leqq M-1,0 \leqq n \leqq N-1) .
\end{aligned}
$$

The key point of Onsager's ingenius method is his observation that the transfer matrix $V$ and the spin operator $s_{m n}$ both belong to the Clifford group $G(W)$ over an orthogonal vector space $W$, which we shall now describe. We introduce operators $p_{m}, q_{m}$ as follows.

$$
\begin{aligned}
p_{m}= & C_{0} C_{1} \cdots C_{m-1} s_{m}, \quad p_{0}=s_{0} \\
q_{m}= & C_{0} C_{1} \cdots C_{m} s_{m}=C_{m} p_{m} \\
& (m=0,1, \ldots, M-1) .
\end{aligned}
$$

By virtue of (5.1.10) we have, for $m, m^{\prime}=0,1, \ldots, M-1$,

$$
p_{m} p_{m^{\prime}}+p_{m^{\prime}} p_{m}=2 \delta_{m m^{\prime}}
$$




$$
\begin{aligned}
& p_{m} q_{m^{\prime}}+q_{m^{\prime}} p_{m}=0 \\
& q_{m} q_{m^{\prime}}+q_{m^{\prime}} q_{m}=-2 \delta_{m m^{\prime}} .
\end{aligned}
$$

In terms of $p_{m}, q_{m}, s_{m}$ and $C_{m}$ are given by

$$
\begin{aligned}
& s_{m}=p_{m} t_{m}, \quad t_{m}=q_{m-1} p_{m-1} \cdots q_{0} p_{0} \\
& C_{m}=q_{m} p_{m} .
\end{aligned}
$$

Now (5.1.16) shows that $W=\bigoplus_{m=0}^{M-1}\left(C p_{m} \oplus C q_{m}\right)$ is equipped with an orthogonal structure, with respect to which the basis $\left\{p_{m}, i q_{m}\right\}_{m=0,1, \ldots, M-1}$ is orthonormal. Since $\operatorname{nr}\left(p_{m}\right), \operatorname{nr}\left(q_{m}\right) \neq 0,(5.1 .17)$ implies that $s_{m}, t_{m} \in G(W)$. Moreover we have from (5.1.8) and (5.1.17)

$$
\begin{aligned}
& V_{1}=\exp \left(K_{1}\left(p_{1} q_{0}+p_{2} q_{1}+\cdots+p_{M-1} q_{M-2}+p_{0} \varepsilon_{w} q_{M-1}\right)\right) \\
& V_{2}=\left(2 \sinh 2 K_{2}\right)^{M / 2} \exp \left(K_{2}^{*}\left(q_{0} p_{0}+q_{1} p_{1}+\cdots+q_{M-1} p_{M-1}\right)\right)
\end{aligned}
$$

where $\varepsilon_{w}=q_{M-1} p_{M-1} \cdots q_{0} p_{0}$ denotes an orientation of $W$ (Chapter I, p. 242).

In the sequel we shall modify the definition of $V_{1}$ as

$$
V_{1}=\exp \left(K_{1}\left(p_{1} q_{0}+p_{2} q_{1}+\cdots+p_{M-1} q_{M-2}+p_{0} q_{M-1}\right)\right)
$$

to avoid complexity, without altering the essence of calculation. This makes the transfer matrix invariant under the horizontal translation $p_{m} \mapsto p_{m+1}, q_{m} \mapsto$ $q_{m+1}$. From (5.1.18) and (5.1.18)' it is clear that $V \in G(W)$.

We fix an expectation value on $A(W)$ given by $\langle a\rangle=Z_{M N}^{-1} \operatorname{trace}\left(a V^{N}\right)=$ trace $\left(a g_{\kappa}\right)(a \in A(W))$, where $g_{\kappa}=V^{N} /$ trace $V^{N} \in G(W)$ (Chapter I, pp. 261 262).

In order to obtain the norms of $s_{m}, t_{m}$ and $V$, let us compute their induced rotations (cf. [8]). We have

$$
\begin{aligned}
& T_{t_{m}} p_{m^{\prime}}= \begin{cases}-p_{m^{\prime}} & \left(0 \leqq m^{\prime} \leqq m-1\right) \\
p_{m^{\prime}} & \left(m \leqq m^{\prime} \leqq M-1\right)\end{cases} \\
& T_{t_{m}} q_{m^{\prime}}= \begin{cases}-q_{m^{\prime}} & \left(0 \leqq m^{\prime} \leqq m-1\right) \\
q_{m^{\prime}} & \left(m \leqq m^{\prime} \leqq M-1\right)\end{cases} \\
& T_{V_{1}^{1 / 2} p_{m}}=p_{m} \cdot \cosh K_{1}-q_{m-1} \cdot \sinh K_{1} \\
& T_{V_{1}^{1 / 2}} q_{m}=-p_{m+1} \cdot \sinh K_{1}+q_{m} \cdot \cosh K_{1} \\
& T_{V_{2}} p_{m}=p_{m} \cdot \cosh 2 K_{2}^{*}+q_{m} \cdot \sinh 2 K_{2}^{*} \\
& T_{V_{2}} q_{m}=p_{m} \cdot \sinh 2 K_{2}^{*}+q_{m} \cdot \cosh 2 K_{2}^{*}
\end{aligned}
$$

where $q_{-1}=q_{M-1}$ and $p_{M}=p_{0}$ in (5.1.20).

If we introduce the Fourier-transformed basis 


$$
\begin{aligned}
\hat{p}\left(\theta_{\mu}\right)= & \sum_{m=0}^{M-1} e^{-i m \theta_{\mu}} p_{m} \\
\hat{q}\left(\theta_{\mu}\right)= & \sum_{m=0}^{M-1} e^{-i m \theta_{\mu}} q_{m} \\
& \left(\theta_{\mu}=2 \pi \mu / M, \mu=0,1, \ldots, M-1 \bmod M\right)
\end{aligned}
$$

the table of inner product becomes

$$
\begin{aligned}
& \left\langle\hat{p}\left(\theta_{\mu}\right), \hat{p}\left(\theta_{v}\right)\right\rangle=2 M \delta_{\mu,-v} \\
& \left\langle\hat{p}\left(\theta_{\mu}\right), \hat{q}\left(\theta_{v}\right)\right\rangle=0 \\
& \left\langle\hat{q}\left(\theta_{\mu}\right), \hat{q}\left(\theta_{v}\right)\right\rangle=-2 M \delta_{\mu,-v}
\end{aligned}
$$

with $\delta_{\mu,-v}=0(\mu \not \equiv-v \bmod M),=1(\mu \equiv-v \bmod M)$, and we have from (5.1.20) and (5.1.21)

$$
\begin{aligned}
& T_{V_{1}^{1 / 2}} \hat{p}\left(\theta_{\mu}\right)=\hat{p}\left(\theta_{\mu}\right) \cdot \cosh K_{1}-\hat{q}\left(\theta_{\mu}\right) \cdot e^{-i \theta_{\mu}} \sinh K_{1} \\
& T_{V_{1}^{1 / 2}} \hat{q}\left(\theta_{\mu}\right)=-\hat{p}\left(\theta_{\mu}\right) \cdot e^{i \theta_{\mu}} \sinh K_{1}+\hat{q}\left(\theta_{\mu}\right) \cdot \cosh K_{1}
\end{aligned}
$$

$$
\begin{aligned}
& T_{V_{2}} \hat{p}\left(\theta_{\mu}\right)=\hat{p}\left(\theta_{\mu}\right) \cdot \cosh 2 K_{2}^{*}+\hat{q}\left(\theta_{\mu}\right) \cdot \sinh 2 K_{2}^{*} \\
& T_{V_{2}} \hat{q}\left(\theta_{\mu}\right)=\hat{p}\left(\theta_{\mu}\right) \cdot \sinh 2 K_{2}^{*}+\hat{q}\left(\theta_{\mu}\right) \cdot \cosh 2 K_{2}^{*} \\
& T_{V} \hat{p}\left(\theta_{\mu}\right)=\hat{p}\left(\theta_{\mu}\right) \cdot \cosh \gamma\left(\theta_{\mu}\right)+\hat{q}\left(\theta_{\mu}\right) \cdot a\left(\theta_{\mu}\right)^{-1} \sinh \gamma\left(\theta_{\mu}\right) \\
& T_{V} \hat{q}\left(\theta_{\mu}\right)=\hat{p}\left(\theta_{\mu}\right) \cdot a\left(\theta_{\mu}\right) \sinh \gamma\left(\theta_{\mu}\right)+\hat{q}\left(\theta_{\mu}\right) \cdot \cosh \gamma\left(\theta_{\mu}\right) .
\end{aligned}
$$

Here $\gamma(\theta)=\gamma(-\theta) \geqq 0$ and $a(\theta)$ are defined by

$$
\begin{aligned}
\cosh \gamma(\theta) & =\cosh 2 K_{1} \cosh 2 K_{2}^{*}-\sinh 2 K_{1} \sinh 2 K_{2}^{*} \cos \theta \\
& =\cosh 2\left(K_{1}-K_{2}^{*}\right)+2 \sinh 2 K_{1} \sinh 2 K_{2}^{*} \sin ^{2}(\theta / 2)
\end{aligned}
$$

$$
\begin{aligned}
a(\theta)^{ \pm 1} \sinh \gamma(\theta)= & 2\left(\cosh K_{1} \cosh K_{2}^{*}-e^{ \pm i \theta} \sinh K_{1} \sinh K_{2}^{*}\right) \\
& \times\left(\cosh K_{1} \sinh K_{2}^{*}-e^{ \pm i \theta} \sinh K_{1} \cosh K_{2}^{*}\right) \\
= & \cosh ^{2} K_{1} \sinh 2 K_{2}^{*}\left(1-\alpha_{1} e^{ \pm i \theta}\right)\left(1-\alpha_{2}^{-1} e^{ \pm i \theta}\right) \\
a(\theta)^{2}= & \frac{\left(1-\alpha_{1} e^{i \theta}\right)\left(1-\alpha_{2}^{-1} e^{i \theta}\right)}{\left(1-\alpha_{1} e^{-i \theta}\right)\left(1-\alpha_{2}^{-1} e^{-i \theta}\right)}
\end{aligned}
$$

with

$$
\begin{aligned}
& \alpha_{1}=\tanh K_{1} \cdot \tanh K_{2}^{*}<1 \\
& \alpha_{2}=\left(\tanh K_{1}\right)^{-1} \tanh K_{2}^{*} .
\end{aligned}
$$

The critical temperature $T=T_{c}$ is defined by the condition

$$
\alpha_{2} \gtreqless 1 \Leftrightarrow T \gtreqless T_{c} .
$$

Notice that for $\theta=0, \gamma(0)=2\left|K_{1}-K_{2}^{*}\right|$ and $a(0)= \pm 1\left(T \gtrless T_{c}\right)$. For $T>T_{c}$, $(5.1 .31)_{T>T_{c}}$

$$
a_{T>T_{c}}(\theta)=a(\theta)=b_{T>T_{c}}(\theta) / b_{T>T_{c}}(-\theta)
$$




$$
b_{T>T_{c}}(\theta)=\sqrt{\left(1-\alpha_{1} e^{i \theta}\right)\left(1-\alpha_{2}^{-1} e^{i \theta}\right)}
$$

is a single-valued function of $z=e^{i \theta}$ on the unit circle $S^{1}=\{|z|=1\}$, while for $T<T_{c}$,

$(5.1 .31)_{T<1_{c}}$

$$
\begin{aligned}
& a_{T<T_{c}}(\theta)=-e^{-i \theta} a(\theta)=b_{T<T_{c}}(\theta) / b_{T<T_{c}}(-\theta) \\
& b_{T<T_{c}}(\theta)=\sqrt{\left(1-\alpha_{1} e^{i \theta}\right) /\left(1-\alpha_{2} e^{i \theta}\right)}
\end{aligned}
$$

enjoys the above property. Here the branch of square root is so chosen that $b_{T \gtrless T_{c}}(0)>0$.

The rotation $T_{V}$ in (5.1.26) is diagonalized in the following basis for $T \gtrless T_{c}$, respectively.

$$
\begin{aligned}
& (5.1 .32)_{T>T_{c}}\left\{\begin{array}{l}
\left.2 \hat{\psi}_{T>T_{c}}^{\dagger}\left(-\theta_{\mu}\right)=\hat{p}\left(\theta_{\mu}\right) \cdot \sqrt{a_{T>T_{c}}\left(\theta_{\mu}\right)}-\hat{q}\left(\theta_{\mu}\right) \cdot \sqrt{a_{T>T_{c}}\left(\theta_{\mu}\right.}\right)^{-1} \\
2 \hat{\psi}_{T>T_{c}}\left(\theta_{\mu}\right)=\hat{p}\left(\theta_{\mu}\right) \cdot \sqrt{a_{T>T_{c}}\left(\theta_{\mu}\right)}+\hat{q}\left(\theta_{\mu}\right) \cdot \sqrt{a_{T>T_{c}}\left(\theta_{\mu}\right)^{-1}}
\end{array}\right. \\
& \left\{\begin{array}{l}
\hat{p}\left(\theta_{\mu}\right)=\hat{\psi}_{T>T_{c}}^{\dagger}\left(-\theta_{\mu}\right) \cdot \sqrt{a_{T>T_{c}}\left(\theta_{\mu}\right)^{-1}}+\hat{\psi}_{T>T_{c}}\left(\theta_{\mu}\right) \cdot \sqrt{a_{T>T_{c}}\left(\theta_{\mu}\right)^{-1}} \\
\hat{q}\left(\theta_{\mu}\right)=-\hat{\psi}_{T>T_{c}}^{\dagger}\left(-\theta_{\mu}\right) \cdot \sqrt{a_{T>T_{c}}\left(\theta_{\mu}\right)}+\hat{\psi}_{T>T_{c}}\left(\theta_{\mu}\right) \cdot \sqrt{a_{T>T_{c}}\left(\theta_{\mu}\right)}
\end{array}\right. \\
& (5.1 .32)_{T<T_{c}}\left\{\begin{array}{l}
2 \hat{\psi}_{T<T_{c}}^{\dagger}\left(-\theta_{\mu}\right)=e^{i \theta_{\mu}} \hat{p}\left(\theta_{\mu}\right) \cdot \sqrt{a_{T<T_{c}}\left(\theta_{\mu}\right)}+\hat{q}\left(\theta_{\mu}\right) \cdot \sqrt{a_{T<T_{c}}\left(\theta_{\mu}\right)^{-1}} \\
2 \hat{\psi}_{T<T_{c}}\left(\theta_{\mu}\right)=e^{i \theta_{\mu}} \hat{p}\left(\theta_{\mu}\right) \cdot \sqrt{a_{T<T_{c}}\left(\theta_{\mu}\right)}-\hat{q}\left(\theta_{\mu}\right) \cdot \sqrt{a_{T<T_{c}}\left(\theta_{\mu}\right)^{-1}}
\end{array}\right. \\
& \left\{\begin{array}{l}
e^{i \theta_{\mu}} \hat{p}\left(\theta_{\mu}\right)=\hat{\psi}_{T<T_{c}}^{\dagger}\left(-\theta_{\mu}\right) \cdot \sqrt{a_{T<T_{c}}\left(\theta_{\mu}\right)^{-1}}+\hat{\psi}_{T<T_{c}}\left(\theta_{\mu}\right) \cdot \sqrt{a_{T<T_{c}}\left(\theta_{\mu}\right)^{-1}} \\
\hat{q}\left(\theta_{\mu}\right)=\hat{\psi}_{T<T_{c}}^{\dagger}\left(-\theta_{\mu}\right) \cdot \sqrt{a_{T<T_{c}}\left(\theta_{\mu}\right)}-\hat{\psi}_{T<T_{c}}\left(\theta_{\mu}\right) \cdot \sqrt{a_{T<T_{c}}\left(\theta_{\mu}\right)} .
\end{array}\right.
\end{aligned}
$$

In either case $\hat{\psi}^{\dagger}\left(\theta_{\mu}\right)=\hat{\psi}_{T \gtrless T_{c}}^{\dagger}\left(\theta_{\mu}\right), \hat{\psi}\left(\theta_{\mu}\right)=\hat{\psi}_{T \gtrless T_{c}}\left(\theta_{\mu}\right)$ satisfy the canonical anticommutation relations

$$
\begin{aligned}
& {\left[\hat{\psi}^{\dagger}\left(\theta_{\mu}\right), \hat{\psi}^{\dagger}\left(\theta_{v}\right)\right]_{+}=0, \quad\left[\hat{\psi}\left(\theta_{\mu}\right), \hat{\psi}\left(\theta_{v}\right)\right]_{+}=0} \\
& {\left[\hat{\psi}^{\dagger}\left(\theta_{\mu}\right), \hat{\psi}\left(\theta_{v}\right)\right]_{+}=M \delta_{\mu \nu}= \begin{cases}M & (\mu \equiv v \bmod M) \\
0 & (\mu \neq v \bmod M),\end{cases} }
\end{aligned}
$$

and we have, using $\gamma(\theta)=\gamma(-\theta)$,

$$
\begin{aligned}
& T_{V} \hat{\psi}^{\dagger}\left(\theta_{\mu}\right)=e^{-\gamma\left(\theta_{\mu}\right)} \hat{\psi}^{\dagger}\left(\theta_{\mu}\right) \\
& T_{V} \hat{\psi}\left(\theta_{\mu}\right)=e^{\gamma\left(\theta_{\mu}\right)} \hat{\psi}\left(\theta_{\mu}\right) \\
& \quad(\mu=0,1, \ldots, M-1 \bmod M)
\end{aligned}
$$

The table $K$ of the expectation value in this basis is computed by applying the formula (1.5.13), i.e. $K=(J+H) / 2, H=J\left(1-T_{V^{N}}\right)\left(1+T_{V^{N}}\right)^{-1}$. We find

$$
\left(\begin{array}{c}
\left\langle\hat{\psi}^{\dagger}\left(\theta_{\mu}\right) \hat{\psi}^{\dagger}\left(\theta_{v}\right)\right\rangle\left\langle\hat{\psi}^{\dagger}\left(\theta_{\mu}\right) \hat{\psi}\left(\theta_{v}\right)\right\rangle \\
\left\langle\hat{\psi}\left(\theta_{\mu}\right) \hat{\psi}^{\dagger}\left(\theta_{v}\right)\right\rangle\left\langle\hat{\psi}\left(\theta_{\mu}\right) \hat{\psi}\left(\theta_{v}\right)\right\rangle
\end{array}\right)=\left(\begin{array}{cc}
0 & \frac{1}{1+e^{N \gamma\left(\theta_{\mu}\right)}} \\
\frac{1}{1+e^{-N \gamma\left(\theta_{\mu}\right)}} & 0
\end{array}\right) M \delta_{\mu v}
$$


Proposition 5.1.1. We have

$$
\begin{aligned}
& \left(2 \sinh 2 K_{2}\right)^{-M / 2} V=\exp (-\mathscr{H}) \\
& \mathscr{H}=\frac{1}{M} \sum_{\mu=0}^{M-1} \gamma\left(\theta_{\mu}\right)\left(\hat{\psi}^{\dagger}\left(\theta_{\mu}\right) \hat{\psi}\left(\theta_{\mu}\right)-\frac{M}{2}\right) .
\end{aligned}
$$

If we set $V^{\prime}=\exp (-\mathscr{H})$, its norm is given by

$$
\begin{aligned}
& \operatorname{Nr}\left(V^{\prime}\right)=\left\langle V^{\prime}\right\rangle e^{\rho^{\prime} / 2} \\
& \left\langle V^{\prime}\right\rangle^{2}=\prod_{\mu=0}^{M-1} \cosh \left((N+1) \gamma\left(\theta_{\mu}\right)\right) \cdot\left(\cosh N \gamma\left(\theta_{\mu}\right)\right)^{-1} \\
& \rho^{\prime} / 2=-\frac{1}{M} \sum_{\mu=0}^{M-1} \frac{\left(1-e^{-\gamma\left(\theta_{\mu}\right)}\right)\left(1+e^{-N \gamma\left(\theta_{\mu}\right)}\right)}{1+e^{-(N+1) \gamma\left(\theta_{\mu}\right)}} \hat{\psi}^{\dagger}\left(\theta_{\mu}\right) \hat{\psi}\left(\theta_{\mu}\right) .
\end{aligned}
$$

Proof. By virtue of the anti-commutation relations (5.1.33), $V^{\prime}$ induces the same rotation as in (5.1.34). It is also clear that $\mathrm{nr}\left(V^{\prime}\right)=V^{\prime} V^{\prime *}=1$. On the other hand, by (5.1.18) and (5.1.18)', the spinorial norms of $V_{1}^{1 / 2}$ and of $\left(2 \sinh 2 K_{2}\right)^{-M / 2} V_{2}$ are easily computed to be 1 . Therefore we have $\left(2 \sinh 2 K_{2}\right)^{-M / 2} V= \pm V^{\prime}$. In order to determine the sign consider the extreme case $K_{1}=0$. In this case $V_{1}=1, a(\theta)=1$, and it is easy to see that the correct choice is the plus sign. This shows (5.1.36). The norm of $V^{\prime}$ is computed directly from the formula (1.5.7), (1.5.8).

\section{Corollary 5.1.2.}

$$
Z_{M N}^{2}=\left(2 \sinh 2 K_{2}\right)^{M N} \cdot \prod_{\mu=0}^{M-1} 2\left(1+\cosh N \gamma\left(\theta_{\mu}\right)\right) .
$$

Proof. Straightforward from the formula (1.5.18).

In the limit $M, N \rightarrow \infty$ (5.1.38) reproduces the celebrated Onsager's formula for the free energy per unit site

$$
\frac{1}{M N} \log Z_{M N} \sim \frac{1}{2} \log \left(2 \sinh 2 K_{2}\right)+\frac{1}{2} \int_{0}^{2 \pi} \frac{d \theta}{2 \pi} \gamma(\theta) .
$$

As has been noted by Onsager, it is rewritten into the symmetrical form $(5.1 .39)^{\prime} \frac{1}{M N} \log Z_{M N}-\log 2 \sim \frac{1}{2} \iint_{0}^{2 \pi} \frac{d \theta}{2 \pi} \frac{d \theta^{\prime}}{2 \pi} \log \left(C_{1} C_{2}-S_{1} \cos \theta-S_{2} \cos \theta^{\prime}\right)$ by using the identity

$$
\gamma=\int_{0}^{2 \pi} \frac{d \theta}{2 \pi} \log (2(\cosh \gamma-\cos \theta)) .
$$

Here we set

$$
C_{i}=\cosh 2 K_{i}, \quad S_{i}=\sinh 2 K_{i}
$$




$$
C_{i}^{*}=\cosh 2 K_{i}^{*}=S_{i}^{-1} C_{i}, S_{i}^{*}=\sinh 2 K_{i}^{*}=S_{i}^{-1} \quad(i=1,2) .
$$

The calculation of $\mathrm{Nr}\left(t_{m}\right), \operatorname{Nr}\left(s_{m}\right)$ will be carried out in the next section.

\section{§5.2. Spin Operators}

Actually the explicit computation of $\mathrm{Nr}\left(s_{m n}\right)$ is performed only in the infinite lattice limit $M, N \rightarrow \infty$. For convenience we replace the lattice size $M, N$ by $2 M+1,2 N+1$ respectively. A lattice site will now be represented as $(m, n)$ with $-M \leqq m \leqq M,-N \leqq n \leqq N$. The spin operators are defined to be $t_{m}=q_{m-1} p_{m-1} \cdots q_{-M} p_{-M}, s_{m}=p_{m} t_{m}$ and $t_{m n}=V^{n} t_{m} V^{-n}, s_{m n}=V^{n} s_{m} V^{-n}$.

In the limit $M, N \rightarrow \infty$ the finite lattice and its Fourier image $\mathbb{Z} /(2 M+1) \mathbb{Z}$ $\times \mathbb{Z} /(2 N+1) \mathbb{Z}$ become $\mathbb{Z}^{2}$ and the torus $(\boldsymbol{R} / 2 \pi \mathbb{Z})^{2}$, respectively. First we fix $M, T\left(\neq T_{c}\right)$ and let $N$ tend $\infty$. The table of expectation values (5.1.35) becomes $^{(*)}$

$$
\left(\begin{array}{c}
\left\langle\hat{\psi}^{\dagger}\left(\theta_{\mu}\right) \hat{\psi}^{\dagger}\left(\theta_{v}\right)\right\rangle\left\langle\hat{\psi}^{\dagger}\left(\theta_{\mu}\right) \hat{\psi}\left(\theta_{v}\right)\right\rangle \\
\left\langle\hat{\psi}\left(\theta_{\mu}\right) \hat{\psi}^{\dagger}\left(\theta_{v}\right)\right\rangle\left\langle\hat{\psi}\left(\theta_{\mu}\right) \hat{\psi}\left(\theta_{v}\right)\right\rangle
\end{array}\right)=\left(\begin{array}{rr} 
& 0 \\
1 &
\end{array}\right)(2 M+1) \delta_{\mu \nu} .
$$

In other words the expectation value $\langle>$ is now the one induced by the holonomic decomposition $W=V^{\dagger} \oplus V, V^{\dagger}=\bigoplus_{\mu=-M}^{M} \mathbb{C} \hat{\psi}^{\dagger}\left(\theta_{\mu}\right), V=\bigoplus_{\mu=-M}^{M} \mathbb{C} \hat{\psi}\left(\theta_{\mu}\right)$.

In view of the simplicity of the rotation $T_{t_{m}}$ in (5.1.19), a convenient basis for the calculation of $\operatorname{Nr}\left(t_{m}\right)$ is $\left\{p_{m}, q_{m}\right\}$ or its Fourier transform $\left\{\hat{p}\left(\theta_{\mu}\right), \hat{q}\left(\theta_{\mu}\right)\right\}$. From (5.2.1) and (5.1.32) ${ }_{T \gtrless T_{c}}$, we have

$$
\begin{aligned}
& \left(\begin{array}{c}
\left\langle\hat{p}\left(\theta_{\mu}\right) \hat{p}\left(\theta_{v}\right)\right\rangle\left\langle\hat{p}\left(\theta_{\mu}\right) \hat{q}\left(\theta_{v}\right)\right\rangle \\
\left\langle\hat{q}\left(\theta_{\mu}\right) \hat{p}\left(\theta_{v}\right)\right\rangle\left\langle\hat{q}\left(\theta_{\mu}\right) \hat{q}\left(\theta_{v}\right)\right\rangle
\end{array}\right) \\
& =\left(\begin{array}{cc}
1 & -a_{T>T_{c}}\left(\theta_{\mu}\right)^{-1} \\
a_{T>T_{c}}\left(\theta_{\mu}\right) & -1
\end{array}\right)(2 M+1) \delta_{\mu,-v} \\
& \left(\begin{array}{l}
\left\langle p_{m} p_{m^{\prime}}\right\rangle\left\langle p_{m} q_{m^{\prime}}\right\rangle \\
\left\langle q_{m} p_{m^{\prime}}\right\rangle\left\langle q_{m} q_{m^{\prime}}\right\rangle
\end{array}\right)=\left(\begin{array}{cc}
\delta_{m m^{\prime}} & -a_{T>T_{c},-m+m^{\prime}}^{(M)} \\
a_{T>T_{c}, T_{-m^{\prime}}}^{(M)} & -\delta_{m m^{\prime}}
\end{array}\right) \\
& (5.2 .2)_{T<T_{c}} \quad\left(\begin{array}{cc}
\left\langle e^{i \theta_{\mu}} \hat{p}\left(\theta_{\mu}\right) e^{i \theta_{v}} \hat{p}\left(\theta_{v}\right)\right\rangle\left\langle e^{i \theta_{\mu}} \hat{p}\left(\theta_{\mu}\right) \hat{q}\left(\theta_{v}\right)\right\rangle \\
\left\langle\hat{q}\left(\theta_{\mu}\right) e^{i \theta_{\nu}} \hat{p}\left(\theta_{v}\right)\right\rangle & \left\langle\hat{q}\left(\theta_{\mu}\right) \hat{q}\left(\theta_{v}\right)\right\rangle
\end{array}\right) \\
& =\left(\begin{array}{cc}
1 & a_{T<T_{c}}\left(\theta_{\mu}\right)^{-1} \\
-a_{T<T_{c}}\left(\theta_{\mu}\right) & -1
\end{array}\right)(2 M+1) \delta_{\mu_{p}-\vartheta} \\
& \left(\begin{array}{l}
\left\langle p_{m} p_{m^{\prime}}\right\rangle\left\langle p_{m} q_{m^{\prime}}\right\rangle \\
\left\langle q_{m} p_{m^{\prime}}\right\rangle\left\langle q_{m} q_{m^{\prime}}\right\rangle
\end{array}\right)=\left(\begin{array}{cc}
\delta_{m m^{\prime}} & a_{T<T_{c},-m+m^{\prime}-1}^{(M)} \\
-a_{T<T_{c}, m-m^{\prime}-1}^{(M)} & -\delta_{m m^{\prime}}
\end{array}\right)
\end{aligned}
$$

(*) In what follows we often drop the subscript $T \gtrless T_{c}$ in case there is no fear of confusion. 
where we have set $a_{T \gtrless T_{c}, m}^{(M)}=(2 M+1)^{-1} \sum_{\mu=-M}^{M} e^{i m \theta_{\mu}} a_{T \gtrless T_{c}}\left(\theta_{\mu}\right)$.

Now we go to the limit $M \rightarrow \infty$. If we set

$$
\begin{aligned}
& P^{0}=\int_{-\pi}^{\pi} \frac{d \theta}{2 \pi} \gamma(\theta) \hat{\psi}^{\dagger}(\theta) \hat{\psi}(\theta) \\
& P^{1}=\int_{-\pi}^{\pi} \frac{d \theta}{2 \pi} \theta \hat{\psi}^{\dagger}(\theta) \hat{\psi}(\theta)
\end{aligned}
$$

the auxiliary operators $p_{m n}=e^{-n P^{0}-i m P^{1}} p_{00} e^{n P^{0}+i m P^{1}}, q_{m n}=e^{-n P^{0}-i m P^{1}} q_{00}$ $\times e^{n P^{0}+i m P^{1}}$ are expressed as

$(5.2 .4)_{T>T_{c}}$

$$
\begin{aligned}
p_{m n}= & \int_{-\pi}^{\pi} \frac{d \theta}{2 \pi}\left(e^{-n \gamma(\theta)-i m \theta} \sqrt{a_{T>T_{c}}(\theta)} \hat{\psi}_{T>T_{c}}^{\dagger}(\theta)\right. \\
& \left.+e^{n \gamma(\theta)+i m \theta} \sqrt{a_{T>T_{c}}(\theta)^{-1}} \hat{\psi}_{T>T_{c}}(\theta)\right) \\
q_{m n}= & \int_{-\pi}^{\pi} \frac{d \theta}{2 \pi}\left(-e^{-n \gamma(\theta)-i m \theta} \sqrt{a_{T>T_{c}}(\theta)}-1 \hat{\psi}_{T>T_{c}}^{\dagger}(\theta)\right. \\
& \left.+e^{n \gamma(\theta)+i m \theta} \sqrt{a_{T>T_{c}}(\theta)} \hat{\psi}_{T>T_{c}}(\theta)\right)
\end{aligned}
$$

$(5.2 .4)_{T<T_{c}} \quad p_{m n}=\int_{-\pi}^{\pi} \frac{d \theta}{2 \pi}\left(e^{-n \gamma(\theta)-i(m-1) \theta} \sqrt{a_{T<T_{c}}(\theta)} \hat{\psi}_{T<T_{c}}^{\dagger}(\theta)\right.$

$$
\begin{aligned}
& \left.+e^{n \gamma(\theta)+i(m-1) \theta} \sqrt{a_{T<T_{c}}(\theta)^{-1}} \hat{\psi}_{T<T_{c}}(\theta)\right) \\
q_{m n}= & \int_{-\pi}^{\pi} \frac{d \theta}{2 \pi}\left(e^{-n \gamma(\theta)-i m \theta} \sqrt{a_{T<T_{c}}(\theta)^{-1}} \hat{\psi}_{T<T_{c}}^{\dagger}(\theta)\right. \\
& \left.-e^{n \gamma(\theta)+i m \theta} \sqrt{a_{T<T_{c}}(\theta)} \hat{\psi}_{T<T_{c}}(\theta)\right) .
\end{aligned}
$$

In this limit (5.2.1) and (5.2.2) ${ }_{T \gtrless T_{c}}$ become respectively

$$
\left(\begin{array}{c}
\left\langle\hat{\psi}^{\dagger}(\theta) \hat{\psi}^{\dagger}\left(\theta^{\prime}\right)\right\rangle\left\langle\hat{\psi}^{\dagger}(\theta) \hat{\psi}\left(\theta^{\prime}\right)\right\rangle \\
\left\langle\hat{\psi}(\theta) \hat{\psi}^{\dagger}\left(\theta^{\prime}\right)\right\rangle\left\langle\hat{\psi}(\theta) \hat{\psi}\left(\theta^{\prime}\right)\right\rangle
\end{array}\right)=\left(\begin{array}{ll} 
& 0 \\
1 &
\end{array}\right) 2 \pi \delta\left(\theta-\theta^{\prime}\right)
$$

$(5.2 .6)_{T>T_{c}}$

$$
\begin{array}{cl}
(5.2 .6)_{T>T_{c}} \quad\left(\begin{array}{l}
\left\langle\hat{p}(\theta) \hat{p}\left(\theta^{\prime}\right)\right\rangle\left\langle\hat{p}(\theta) \hat{q}\left(\theta^{\prime}\right)\right\rangle \\
\left\langle\hat{q}(\theta) \hat{p}\left(\theta^{\prime}\right)\right\rangle\left\langle\hat{q}(\theta) \hat{q}\left(\theta^{\prime}\right)\right\rangle
\end{array}\right) \\
=\left(\begin{array}{cc}
1 & -a_{T>T_{c}}(\theta)^{-1} \\
a_{T>T_{c}}(\theta) & -1
\end{array}\right) 2 \pi \delta\left(\theta+\theta^{\prime}\right) \\
\left(\begin{array}{cc}
\left\langle p_{m} p_{m^{\prime}}\right\rangle\left\langle p_{m} q_{m^{\prime}}\right\rangle \\
\left\langle q_{m} p_{m^{\prime}}\right\rangle\left\langle q_{m} q_{m^{\prime}}\right\rangle
\end{array}\right)=\left(\begin{array}{cc}
\delta_{m m^{\prime}} & -a_{T>T_{c},-m+m^{\prime}} \\
a_{T>T_{c}, m-m^{\prime}} & -\delta_{m m^{\prime}}
\end{array}\right) \\
(5.2 .6)_{T<T_{c}} \quad\left(\begin{array}{cc}
\left\langle e^{i \theta} \hat{p}(\theta) e^{i \theta^{\prime}} \hat{p}\left(\theta^{\prime}\right)\right\rangle\left\langle e^{i \theta} \hat{p}(\theta) \hat{q}\left(\theta^{\prime}\right)\right\rangle \\
\left\langle\hat{q}(\theta) e^{i \theta^{\prime}} \hat{p}\left(\theta^{\prime}\right)\right\rangle & \left\langle\hat{q}(\theta) \hat{q}\left(\theta^{\prime}\right)\right\rangle
\end{array}\right)
\end{array}
$$

$$
\begin{gathered}
=\left(\begin{array}{cc}
1 & a_{T<T_{c}}(\theta)^{-1} \\
-a_{T<T_{c}}(\theta) & -1
\end{array}\right) 2 \pi \delta\left(\theta+\theta^{\prime}\right) \\
\left(\begin{array}{c}
\left\langle p_{m} p_{m^{\prime}}\right\rangle\left\langle p_{m} q_{m^{\prime}}\right\rangle \\
\left\langle q_{m} p_{m^{\prime}}\right\rangle\left\langle q_{m} q_{m^{\prime}}\right\rangle
\end{array}\right)=\left(\begin{array}{cc}
\delta_{m m^{\prime}} & a_{T<T_{c},-m+m^{\prime}-1} \\
-a_{T<T_{c}, m^{-m^{\prime}-1}} & -\delta_{m m^{\prime}}
\end{array}\right)
\end{gathered}
$$


where $a_{T \gtrless T_{c}}(\theta)=\sum_{m=-\infty}^{+\infty} e^{-i m \theta} a_{T \gtrless T_{c}, m}$.

We distinguish the two cases $T>T_{c}$ and $T<T_{c}$.

(i) The case $T>T_{c}$.

We shall first consider the even element $t_{m 0}=t_{m 0}^{(M)}$ on the lattice of size $(2 M+1) \times \infty$. From the formula (1.5.7) (or directly from (A.26)' of [3]) we have

$$
\left\langle t_{00}^{(M)} t_{m 0}^{(M)}\right\rangle=\operatorname{det}\left(\begin{array}{ccc}
a_{0}^{(M)} & \cdots & a_{-m+1}^{(M)} \\
\vdots & \ddots & \vdots \\
a_{m-1}^{(M)} & \cdots & a_{0}^{(M)}
\end{array}\right)
$$

where $a_{m}^{(M)}=a_{T>T_{c}, m}^{(M)}$. Now we let $M \rightarrow \infty$ ( $m$ fixed $)$ and obtain in the infinite lattice

$$
\left\langle t_{00} t_{m 0}\right\rangle=\operatorname{det}\left(\begin{array}{ccc}
a_{0} & \cdots & a_{-m+1} \\
\vdots & \ddots & \vdots \\
a_{m-1} & \cdots & a_{0}
\end{array}\right) .
$$

Finally we take the limit $m \rightarrow \infty$. The right hand side of (5.2.8) in this limit is evaluated by appealing to Szego's theorem ([17]). Using the fact that $\left\langle t_{00} t_{m 0}\right\rangle$ $\rightarrow\left\langle t_{00}\right\rangle^{2}$ as $m \rightarrow \infty$ we obtain

$$
\left\langle t_{00}\right\rangle=\left(1-S_{1}^{2} S_{2}^{2}\right)^{1 / 8}\left(\cosh K_{1}\right)^{-1}
$$

in the infinite lattice, where $S_{1}, S_{2}$ are given in (5.1.40).

In particular $t_{00}^{(M)} \neq 0$ for sufficiently large $M$. This implies that the norm of $t_{00}^{(M)}$ has the form

$$
\begin{aligned}
& \operatorname{Nr}\left(t_{00}^{(M)}\right)=\left\langle t_{00}^{(M)}\right\rangle e^{\hat{\rho}_{00}^{(M)} / 2} \\
& \hat{\rho}_{00}^{(M)}=\left(\frac{1}{2 M+1}\right)^{2} \sum_{\mu, v=-M}^{M}\left(\hat{p}\left(\theta_{\mu}\right) \hat{q}\left(\theta_{\mu}\right)\right) \hat{R}^{(M)}\left(\theta_{\mu}, \theta_{v}\right)\left(\begin{array}{l}
\hat{p}\left(\theta_{v}\right) \\
\hat{q}\left(\theta_{v}\right)
\end{array}\right)
\end{aligned}
$$

where $R^{(M)} \in \operatorname{End}_{C}(W)$ corresponding to $\left(\hat{R}^{(M)}\left(\theta_{\mu}, \theta_{v}\right)\right)_{\mu, v=-M, \ldots, M}$ is related to $P^{(M)}, E^{(M)}$ through (cf. (1.5.8))

$$
\begin{aligned}
R^{(M)} J^{(M)} & =\left(T^{(M)}-1\right)\left(K^{(M)}+{ }^{t} K^{(M)} T^{(M)}\right)^{-1} J^{(M)} \\
& =-2 P^{(M)}\left(\left(1-P^{(M)}\right)+E^{(M)} P^{(M)}\right)^{-1} .
\end{aligned}
$$

In the limit $M \rightarrow \infty$ the operators $P^{(M)}, E^{(M)}$ become

$$
\begin{aligned}
& \left(P \hat{p}\left(\theta^{\prime}\right), P \hat{q}\left(\theta^{\prime}\right)\right)=\int_{-\pi}^{\pi} \frac{d \theta}{2 \pi}(\hat{p}(\theta), \hat{q}(\theta)) P\left(\theta, \theta^{\prime}\right) \\
& P\left(\theta, \theta^{\prime}\right)=I_{2} \cdot \sum_{m=-\infty}^{-1} e^{i m\left(\theta-\theta^{\prime}\right)}=I_{2} \cdot \frac{e^{-i\left(\theta-\theta^{\prime}\right)}}{1-e^{-i\left(\theta-\theta^{\prime}-i 0\right)}}
\end{aligned}
$$




$$
\begin{aligned}
& (E \hat{p}(\theta), E \hat{q}(\theta))=(\hat{p}(\theta), \hat{q}(\theta)) E(\theta) \\
& E(\theta)=-\left(\begin{array}{ll}
a_{T>T_{c}}(\theta) \\
a_{T>T_{c}}(-\theta)
\end{array}\right.
\end{aligned}
$$

Consider the factorization of $E(\theta)$ given by

$$
\begin{aligned}
& X_{-}(\theta)=X_{+}(\theta) E(\theta) \\
& X_{+}(\theta)=\left(\begin{array}{ll}
b_{T>T_{c}}(\theta)^{-1} & \\
& b_{T>T_{c}}(\theta)
\end{array}\right), \\
& X_{-}(\theta)=-\left(\begin{array}{cc}
b_{T>T_{c}}(-\theta)^{-1} \\
b_{T>T_{c}}(-\theta)
\end{array}\right.
\end{aligned}
$$

Clearly $X_{ \pm}(\theta)$ is holomorphic and invertible on $|z|^{ \pm 1} \leqq 1\left(z=e^{i \theta}\right)$. This implies that

$$
P X_{+}^{ \pm 1}(1-P)=0, \quad(1-P) X^{ \pm 1} P=0 .
$$

Therefore by applying (A.18) $)_{F}-(\mathrm{A} .19)_{F}$ in Chapter IV we obtain $R=$ $-2 X_{-}^{-1} \cdot P X_{+} J^{-1}$; namely the corresponding kernel $\hat{R}^{\prime}\left(\theta, \theta^{\prime}\right)$ in the basis $\{\hat{p}(\theta), \hat{q}(\theta)\}$ is given by

$$
\hat{R}^{\prime}\left(\theta, \theta^{\prime}\right)=\frac{e^{-i\left(\theta+\theta^{\prime}\right)}}{1-e^{-i\left(\theta+\theta^{\prime}-i 0\right)}}\left(\begin{array}{ll}
-\frac{b_{T>T_{c}}\left(-\theta^{\prime}\right)}{b_{T>T_{c}}(-\theta)} \\
\frac{b_{T>T_{c}}(-\theta)}{b_{T>T_{c}}\left(-\theta^{\prime}\right)}
\end{array}\right) .
$$

Remark. It is easy to verify that $P, E$ and $X_{ \pm}$are bounded linear operators on $\left(L^{2}\left(S^{1}\right)\right)^{2}$. Hence $K+{ }^{t} K T=J(1-P+E P)$ has a unique inverse $\left(X_{+}^{-1}(1-P)\right.$ $\left.+X_{-}^{-1} P\right) X_{+} J^{-1}$ in $L^{2}$ according to the Remark below Proposition A.2, Chapter IV.

For general $(m, n)$, the rotation $T_{t_{m n}}=1-2 P_{m n}$ induced by $t_{m n}$ is obtained by the replacement $P\left(\theta, \theta^{\prime}\right) \mapsto P_{m n}\left(\theta, \theta^{\prime}\right)=U_{m n}(\theta) P\left(\theta, \theta^{\prime}\right) U_{m n}\left(\theta^{\prime}\right)^{-1}$ with $U_{m n}(\theta)=$ $e^{i m \theta}(\cosh n \gamma(\theta)-E(\theta) \sinh n \gamma(\theta))$. Since $U_{m n}$ commutes with $E,(5.2 .14)$ and (5.2.15) are valid if we replace $X_{ \pm}$by $U_{m n} X_{ \pm} U_{m n}^{-1}$. It is easy to see that the expectation value $\left\langle t_{m n}\right\rangle$ is not changed. Returning to the basis $\hat{\psi}^{\dagger}(\theta), \hat{\psi}(\theta)$ we have thus the following result.

$$
\begin{aligned}
& \operatorname{Nr}\left(t_{m n}\right)=\left\langle t_{m n}\right\rangle e^{\rho_{m n} / 2} \\
& \rho_{m n}=\iint_{-\pi}^{\pi} \frac{d \theta}{2 \pi} \frac{d \theta^{\prime}}{2 \pi}\left(\hat{\psi}^{\dagger}(\theta) \hat{\psi}(\theta)\right)\left(\begin{array}{ll}
\hat{R}_{m n}^{--}\left(\theta, \theta^{\prime}\right) & \hat{R}_{m n}^{-+}\left(\theta, \theta^{\prime}\right) \\
\hat{R}_{m n}^{+-}\left(\theta, \theta^{\prime}\right) & \hat{R}_{m n}^{++}\left(\theta, \theta^{\prime}\right)
\end{array}\right)\left(\begin{array}{l}
\hat{\psi}^{\dagger}\left(\theta^{\prime}\right) \\
\hat{\psi}\left(\theta^{\prime}\right)
\end{array}\right)
\end{aligned}
$$

where $\left\langle t_{m n}\right\rangle=\left\langle t_{00}\right\rangle$ is given by (5.2.9), and 


$$
\begin{aligned}
\hat{R}_{m n}^{\sigma \sigma^{\prime}}\left(\theta, \theta^{\prime}\right)= & \left(\sigma \frac{|b(\theta)|}{\left|b\left(\theta^{\prime}\right)\right|}-\sigma^{\prime} \frac{\left|b\left(\theta^{\prime}\right)\right|}{|b(\theta)|}\right) \\
& \times \frac{e^{i(m-1)\left(\sigma \theta+\sigma^{\prime} \theta^{\prime}\right)+n\left(\sigma \gamma(\theta)+n^{\prime} \gamma\left(\theta^{\prime}\right)\right)}}{1-e^{-i\left(\sigma \theta+\sigma^{\prime} \theta^{\prime}-i 0\right)}} \\
(b(\theta) & \left.=b_{T>T_{c}}(\theta) ; \sigma, \sigma^{\prime}= \pm\right) .
\end{aligned}
$$

Computation of $\operatorname{Nr}\left(s_{m n}\right)$ is now a relatively easy task. Since $s_{m n}=p_{m n} \cdot t_{m n}$ we have from (5.2.17) and (1.4.1)

$$
\begin{aligned}
& \operatorname{Nr}\left(s_{m n}\right)=\left\langle t_{m n}\right\rangle \hat{\psi}_{0, m n} e^{\rho_{m n} / 2} \\
& \hat{\psi}_{0, m n}=\int_{-\pi}^{\pi} \frac{d \theta}{2 \pi} \frac{1}{|b(\theta)|}\left(e^{-i m \theta-n \gamma(\theta)} \hat{\psi}^{\dagger}(\theta)+e^{i m \theta+n \gamma(\theta)} \hat{\psi}(\theta)\right) .
\end{aligned}
$$

Here we have used

$$
\begin{aligned}
& \int_{-\pi}^{\pi} \frac{d \theta^{\prime}}{2 \pi} \hat{R}_{m n}^{\mp-}\left(\theta, \theta^{\prime}\right) \sqrt{a\left(\theta^{\prime}\right)^{-1}} e^{i m \theta^{\prime}+n \gamma\left(\theta^{\prime}\right)} \\
& =e^{\mp(i m \theta+n \gamma(\theta))}\left(\sqrt{a(\theta)} \pm 1-\frac{1}{|b(\theta)|}\right) .
\end{aligned}
$$

(ii) The case $T<T_{c}$

In this case we shall deal with the operator $\bar{s}_{m n}=V^{n} \bar{s}_{m} V^{-n}, \bar{s}_{m}=s_{-M}^{-1} s_{m}=$ $p_{m} q_{m-1} p_{m-1} q_{m-2} \cdots p_{-M+1} q_{-M}$, instead of $s_{m n}$ itself. This amounts to setting the boundary condition $\bar{s}_{-M}=1$ before taking the limit $M \rightarrow \infty$.

More precisely we start with the following:

$$
Z_{M N}^{\prime}=\sum_{(\sigma)}^{\prime} e^{-\beta E(\sigma)}
$$

$$
\rho_{k}^{\prime}\left(\left(m_{1}, n_{1}\right), \ldots,\left(m_{k}, n_{k}\right)\right)=Z_{M N}^{\prime}{ }^{-1} \sum_{(\sigma)}^{\prime} \sigma_{m_{1} n_{1}} \cdots \sigma_{m_{k} n_{k}} e^{-\beta E(\sigma)}
$$

where $\sum_{(\sigma)}^{\prime}$ stands for the sum with the restriction $\sigma_{0 n}=1(0 \leqq n \leqq N-1)$. Following the procedure of Section 5.1 we find

$$
\begin{array}{ll}
(5.1 .12)^{\prime} & Z_{M N}^{\prime}=2^{-1} \operatorname{trace}\left(V^{\prime N}\right) \\
(5.1 .13)^{\prime} & \rho_{k}^{\prime}\left(\left(m_{1}, n_{1}\right), \ldots,\left(m_{k}, n_{k}\right)\right)=\left(2 Z_{M N}^{\prime}\right)^{-1} \operatorname{trace}\left(\bar{s}_{m_{1} n_{1}} \cdots \bar{s}_{m_{k} n_{k}} V^{\prime N}\right)
\end{array}
$$

where

$$
\begin{aligned}
(5.1 .11)^{\prime} & V^{\prime}=V_{1}^{\prime \frac{1}{2}} V_{2}^{\prime} V_{1}^{\prime \frac{1}{2}} \\
V_{1}^{\prime} & =\exp \left(K_{1}\left(p_{1} q_{0}+p_{2} q_{1}+\cdots+p_{0} \varepsilon_{W} q_{M-1}\right)\right) \\
V_{2}^{\prime} & =e^{K_{2}}\left(2 \sinh 2 K_{2}\right)^{\frac{M-1}{2}} \exp \left(K_{2}^{*}\left(q_{1} p_{1}+\cdots+q_{M-1} p_{M-1}\right)\right) \\
(5.1 .14)^{\prime} & \bar{s}_{m n}=V^{\prime n} \bar{s}_{m} V^{\prime-n}, \quad \bar{s}_{m}=p_{m} q_{m-1} \cdots p_{1} q_{0}
\end{aligned}
$$

and $p_{m}, q_{m}$ satisfy (5.1.16). If we replace $V_{1}^{\prime}, V_{2}^{\prime}$ by those given in (5.1.18) and (5.1.18)' respectively, we return to the situation described above. It is not 
difficult to verify that this replacement does not affect the result in the limit $M, N \rightarrow \infty$.

Calculation of $\operatorname{Nr}\left(\bar{s}_{m n}\right)$ is quite parallel to the case of $t_{m n}$ by using the basis $\left\{e^{i \theta} \hat{p}(\theta), \hat{q}(\theta)\right\}$. Explicitly the rotation $T_{\bar{s}_{00}}=1-2 \bar{P}$ reads

$$
\bar{P}\left(\theta, \theta^{\prime}\right)=I_{2} \cdot \frac{e^{-i\left(\theta-\theta^{\prime}\right)}}{1-e^{-i\left(\theta-\theta^{\prime}-i 0\right)}} .
$$

Accordingly (5.2.13), (5.2.14) and (5.2.16) are replaced by

$$
\begin{gathered}
\bar{E}\left(\theta, \theta^{\prime}\right)=\left(\begin{array}{ll} 
& a_{T<T_{c}}(\theta) \\
a_{T<T_{c}}(-\theta) &
\end{array}\right) \\
\bar{X}_{+}(\theta)=\left(\begin{array}{ll}
b_{T<T_{c}}(\theta)^{-1} & b_{T<T_{c}}(\theta)
\end{array}\right) \\
\bar{X}_{-}(\theta)=\left(\begin{array}{ll}
b_{T<T_{c}}(-\theta)^{-1} \\
b_{T<T_{c}}(-\theta) & -\frac{b_{T<T_{c}}\left(-\theta^{\prime}\right)}{b_{T<T_{c}}(-\theta)}
\end{array}\right) \\
\bar{R}^{\prime}\left(\theta, \theta^{\prime}\right)=-\frac{e^{-i\left(\theta+\theta^{\prime}\right)}}{1-e^{-i\left(\theta+\theta^{\prime}-i 0\right)}}\left(\begin{array}{l}
b_{T<T_{c}}(-\theta) \\
b_{T<T_{c}}\left(-\theta^{\prime}\right)
\end{array}\right.
\end{gathered}
$$

respectively.

As a result we have

$$
\begin{aligned}
& \operatorname{Nr}\left(\bar{s}_{m n}\right)=\left\langle\bar{s}_{m n}\right\rangle e^{\bar{\rho}_{m n} / 2} \\
& \begin{aligned}
\bar{\rho}_{m n}=\iint_{-\pi}^{\pi} \frac{d \theta}{2 \pi} \frac{d \theta^{\prime}}{2 \pi}\left(\hat{\psi}^{\dagger}(\theta) \hat{\psi}(\theta)\right) \\
\times\left(\begin{array}{cc}
\bar{R}_{m n}^{--}\left(\theta, \theta^{\prime}\right) & \bar{R}_{m n}^{-+}\left(\theta, \theta^{\prime}\right) \\
\bar{R}_{m n}^{+-}\left(\theta, \theta^{\prime}\right) & \bar{R}_{m n}^{++}\left(\theta, \theta^{\prime}\right)
\end{array}\right)\left(\begin{array}{c}
\hat{\psi}^{\dagger}\left(\theta^{\prime}\right) \\
\hat{\psi}\left(\theta^{\prime}\right)
\end{array}\right)
\end{aligned}
\end{aligned}
$$

where $\hat{\psi}^{\dagger}(\theta)=\hat{\psi}_{T<T_{c}}^{\dagger}(\theta), \hat{\psi}(\theta)=\hat{\psi}_{T<T_{c}}(\theta)$ and

$$
\begin{aligned}
\left\langle\bar{s}_{m n}\right\rangle=(1- & \left.S_{1}^{-2} S_{2}^{-2}\right)^{1 / 8} \\
\bar{R}_{m n}^{\sigma \sigma^{\prime}}\left(\theta, \theta^{\prime}\right)= & \left(\sigma \frac{|b(\theta)|}{\left|b\left(\theta^{\prime}\right)\right|}-\sigma^{\prime} \frac{\left|b\left(\theta^{\prime}\right)\right|}{|b(\theta)|}\right) \\
& \times \frac{e^{i(m-1)\left(\sigma \theta+\sigma^{\prime} \theta^{\prime}\right)+n\left(\sigma \gamma(\theta)+\sigma^{\prime} \gamma\left(\theta^{\prime}\right)\right)}}{1-e^{-i\left(\sigma \theta+\sigma^{\prime} \theta^{\prime}-i 0\right)}} \\
(b(\theta)= & \left.b_{T<T_{c}}(\theta) ; \sigma, \sigma^{\prime}= \pm\right) .
\end{aligned}
$$

In Chapter 4 we constructed the operator $\varphi_{F}(a)$ starting from the 2-dimensional Dirac equation. Likewise we can begin with the following difference equation for $v=\left(v_{m n}\right)_{m, n \in Z}, v_{m n}={ }^{t}\left(v_{m n}^{(+)}, v_{m n}^{(-)}\right) \in \boldsymbol{C}^{2}$. 


$$
\begin{aligned}
v_{m n+1}^{(+)}= & C_{1} C_{2}^{*} v_{m n}^{(+)}-S_{1} S_{2}^{*} \frac{v_{m-1 n}^{(+)}+v_{m+1 n}^{(+)}}{2} \\
& +C_{1}+1-S_{2}^{*} v_{m n}^{(-)}-S_{1} C_{2}^{*} v_{m-1 n}^{(-)}+C_{1}-1 \\
2 & S_{2}^{*} v_{m-2 n}^{(-)} \\
v_{m n+1}^{(-)}= & \frac{C_{1}+1}{2} S_{2}^{*} v_{m n}^{(+)}-S_{1} C_{2}^{*} v_{m+1 n}^{(+)}+\frac{C_{1}-1}{2}-S_{2}^{*} v_{m+2 n}^{(+)} \\
& +C_{1} C_{2}^{*} v_{m n}^{(-)}-S_{1} S_{2}^{*} \frac{v_{m-1 n}^{(-)}+v_{m+1 n}^{(-)}}{2}
\end{aligned}
$$

We denote by $W^{\prime}$ the set of solutions of (5.2.27) satisfying

$$
\sum_{m \in Z}\left|v_{m n}^{(+)}\right|^{2}+\sum_{m \in Z}\left|v_{m n}^{(-)}\right|^{2}<\infty
$$

for a fixed $n$. The inner product in $W^{\prime}$ is defined by

$$
\left\langle v, v^{\prime}\right\rangle=2 \sum_{m \in \boldsymbol{Z}}\left(v_{m n}^{(+)} v_{m n}^{(+) \prime}-v_{m n}^{(-)} v_{m n}^{(-) \prime}\right) .
$$

A little computation shows that the right hand side is independent of $n$.

From (5.1.16) and (5.2.28) we know that if we identify $p_{m_{0}}$ (resp. $\left.q_{m_{0}}\right) \in W$ with the solution $v$ of (5.2.27) satisfying $v_{m 0}={ }^{t}\left(\delta_{m m_{0}}, 0\right)\left(\right.$ resp. $\left.v_{m 0}={ }^{t}\left(0,-\delta_{m m_{0}}\right)\right)$, $W$ and $W^{\prime}$ are isomorphic as orthogonal vector spaces. Moreover, from (5.1.24) and (5.1.25) $p_{m_{0} n_{0}}$ (resp. $q_{m_{0} n_{0}}$ ) represents the solution $v$ such that $v_{m n_{0}}={ }^{t}\left(\delta_{m m_{0}}, 0\right)$ (resp. $v_{m n_{0}}={ }^{t}\left(0,-\delta_{m m_{0}}\right)$ ).

Let us introduce "the mass shell" for the difference equation (5.2.27). Denoting by $z$ and $w$ the translations

$$
\left(z v^{( \pm)}\right)_{m n}=v_{m+1 n}^{( \pm)}, \quad\left(w v^{( \pm)}\right)=v_{m n+1}^{( \pm)},
$$

respectively, we can rewrite $(5.2 .27)$ in the form

$$
(5.2 .27)^{\prime} \quad \Gamma v=0
$$

where

$$
\Gamma=\left(\begin{array}{cc}
C_{1} C_{2}^{*}-S_{1} S_{2}^{*} \frac{z+z^{-1}}{2}-w & \frac{C_{1}+1}{2} S_{2}^{*}-S_{1} C_{2}^{*} z^{-1}+\frac{C_{1}-1}{2} S_{2}^{*} z^{-2} \\
\frac{C_{1}+1}{2} S_{2}^{*}-S_{1} C_{2}^{*} z+\frac{C_{1}-1}{2} S_{2}^{*} z^{2} & C_{1} C_{2}^{*}-S_{1} S_{2}^{*} z+\frac{z^{-1}}{2}-w
\end{array}\right) .
$$

Noting that $C_{2}^{*} S_{2}=C_{2}$ and $S_{2}^{*} S_{2}=1$, we have $\operatorname{det} \Gamma=w^{2}-2\left(C_{1} C_{2}^{*}-\right.$ $\left.S_{1} S_{2}^{*} \cdot \frac{z+z^{-1}}{2}\right) w+1=-2 S_{2}^{*} w \Delta(z, w)$ where

$$
\Delta(z, w)=C_{1} C_{2}-S_{1} \cdot \frac{z+z^{-1}}{2}-S_{2} \cdot \frac{w+w^{-1}}{2} .
$$

We denote by $M^{\boldsymbol{c}}$ the complex mass shell 


$$
\begin{aligned}
M^{\boldsymbol{c}}=\{ & \left(\zeta_{0}, \zeta_{1}, \zeta_{2}\right) \in P^{2}(\boldsymbol{C}) \mid \\
& \left.C_{1} C_{2} \zeta_{0} \zeta_{1} \zeta_{2}-S_{1} \zeta_{2} \cdot \frac{\zeta_{1}^{2}+\zeta_{0}^{2}}{2}-S_{2} \zeta_{1} \cdot \frac{\zeta_{2}^{2}+\zeta_{0}^{2}}{2}=0\right\}
\end{aligned}
$$

$M^{\boldsymbol{C}}$ is a non-singular elliptic curve. The projection $\pi_{1}: M^{\boldsymbol{c}} \rightarrow P^{1}(\boldsymbol{C}), \pi_{1}\left(\zeta_{0}, \zeta_{1}\right.$, $\left.\zeta_{2}\right)=\left(\zeta_{0}, \zeta_{1}\right)$ is a two-sheeted covering with branch points $\alpha_{1}^{ \pm 1}, \alpha_{2}^{ \pm 1}$ (see (5.1.25) and (5.1.26)). We set

$$
\begin{aligned}
& M=\left\{(z, w) \in M^{c}|| z \mid=1\right\}, \\
& M_{ \pm}=\{(z, w) \in M|| w \mid \gtrless 1\} .
\end{aligned}
$$

An Abelian differential of the first kind on $M^{\boldsymbol{C}}$ is given by

$$
\frac{d z}{\pi i z\left(w-w^{-1}\right)}=\frac{d z}{\pi i S_{1} S_{2}^{*} \sqrt{\left(z-\alpha_{1}\right)\left(z-\alpha_{1}^{-1}\right)\left(z-\alpha_{2}\right)\left(z-\alpha_{2}^{-1}\right)}} .
$$

We choose a uniformizing parameter $U$ on $M^{\boldsymbol{c}}$ so that $d U=d z / \pi i z\left(w-w^{-1}\right)$. We identify $M_{ \pm}$with $\boldsymbol{R} / 2 \pi \boldsymbol{Z}$ by

$$
\begin{array}{ll}
z=e^{i \theta}, \quad w=e^{\gamma(\theta)} & \text { if } \quad(z, w) \in M_{+}, \\
z=e^{-i \theta}, \quad w=e^{-\gamma(\theta)} & \text { if } \quad(z, w) \in M_{-},
\end{array}
$$

respectively. Then on the real mass shell $M$ the 1 -form $d U$ is expressed as

$$
d U=\underline{d \theta} \overline{\overline{d e f}} \frac{d \theta}{2 \pi \sinh \gamma(\theta)} .
$$

For a function $f(U)$ defined on $M$ we have the following identities.

$$
\int_{M} d U f(U)=\int_{-\pi}^{\pi} \underline{d \theta} f\left(U_{+}(\theta)\right)+\int_{-\pi}^{\pi} d \theta f\left(U_{-}(\theta)\right),
$$

where $U_{ \pm}(\theta)=\left(e^{ \pm i \theta}, e^{ \pm \gamma(\theta)}\right)$ for $\theta \in \boldsymbol{R} / 2 \pi \boldsymbol{Z}$.

Set for $T>T_{c}$

$$
\begin{array}{ll}
\psi^{\dagger}(\theta)=\sqrt{\sinh \gamma(\theta)} \hat{\psi}_{T>T_{c}}^{\dagger}(\theta) & U \in M_{-}, \\
\psi(\theta)=\sqrt{\sinh \gamma(\theta)} \hat{\psi}_{T>T_{c}}(\theta) & U \in M_{+},
\end{array}
$$

and set for $T<T_{c}$

$$
\begin{array}{ll}
\psi^{\dagger}(\theta)=i \sqrt{\sinh \gamma(\theta)} \hat{\psi}_{T<T_{c}}^{\dagger}(\theta) & U \in M_{-}, \\
\psi(\theta)=-i \sqrt{\sinh \gamma(\theta)} \hat{\psi}_{T<T_{c}}(\theta) & U \in M_{+} .
\end{array}
$$

We have then

(*) We use $z=\zeta_{1} / \zeta_{\mathrm{o}}$ and $w=\zeta_{2} / \zeta_{\mathrm{o}}$ as the inhomogeneous coordinates. 


$$
\left(\begin{array}{ll}
\left\langle\psi^{\dagger}(\theta) \psi^{\dagger}\left(\theta^{\prime}\right)\right\rangle & \left\langle\psi^{\dagger}(\theta) \psi\left(\theta^{\prime}\right)\right\rangle \\
\left\langle\psi(\theta) \psi^{\dagger}\left(\theta^{\prime}\right)\right\rangle & \left\langle\psi(\theta) \psi\left(\theta^{\prime}\right)\right\rangle
\end{array}\right)=\left(\begin{array}{ll} 
& 0 \\
1 &
\end{array}\right) \underline{\delta}\left(\theta, \theta^{\prime}\right),
$$

where $\underline{\delta}\left(\theta, \theta^{\prime}\right)=\sinh \gamma(\theta) \cdot 2 \pi \delta\left(\theta-\theta^{\prime}\right)$. We also set

$$
\psi(U)= \begin{cases}\psi^{\dagger}(\theta) & U \in M_{-}, \\ \psi(\theta) & U \in M_{+} .\end{cases}
$$

From (5.1.28) and (5.1.31) we have the following identities.

$$
\begin{aligned}
& \left|b_{T>T_{c}}(\theta)\right|^{2}=\sinh \gamma(\theta) \sinh 2 K_{2} / \cosh ^{2} K_{1}, \\
& \left|b_{T<T_{c}}(\theta)\right|^{2}=\tanh K_{1} / \tanh (\gamma(\theta) / 2) .
\end{aligned}
$$

Making use of (5.2.39) we obtain the final form of the spin operators.

Theorem 5.2.1. For $T>T_{c}$,

$$
\begin{aligned}
& \operatorname{Nr}\left(s_{m n}\right)=\left(1-S_{1}^{2} S_{2}^{2}\right)^{1 / 8} \psi_{0, m n} e^{\rho_{m n} / 2} . \\
& \rho_{m n}=\iint_{-\pi}^{\pi} d \theta \underline{d \theta^{\prime}}\left(\psi^{\dagger}(\theta) \psi(\theta)\right)\left(\begin{array}{ll}
R_{m n}^{--}\left(\theta, \theta^{\prime}\right) & R_{m n}^{-+}\left(\theta, \theta^{\prime}\right) \\
R_{m n}^{+-}\left(\theta, \theta^{\prime}\right) & R_{m n}^{++}\left(\theta, \theta^{\prime}\right)
\end{array}\right)\left(\begin{array}{l}
\psi^{\dagger}\left(\theta^{\prime}\right) \\
\psi\left(\theta^{\prime}\right)
\end{array}\right) \\
& \quad=\iint_{M \times M} d U d U^{\prime} R_{m n}\left(U, U^{\prime}\right) \psi(U) \psi\left(U^{\prime}\right), \\
& R_{m n}^{\sigma \sigma^{\prime}}\left(\theta, \theta^{\prime}\right)=\frac{\sinh \sigma \gamma(\theta)-\sinh \sigma^{\prime} \gamma\left(\theta^{\prime}\right)}{1-e^{-i\left(\sigma \theta+\sigma^{\prime} \theta^{\prime}-i 0\right)}} \\
& \quad \times e^{i(m-1)\left(\sigma \theta+\sigma^{\prime} \theta^{\prime}\right)+n\left(\sigma \gamma(\theta)+\sigma^{\prime} \gamma\left(\theta^{\prime}\right)\right)}\left(\sigma, \sigma^{\prime}= \pm\right), \\
& R_{m n}\left(U, U^{\prime}\right)=\frac{w-w^{\prime}}{1-z^{-1} z^{\prime-1}} \cdot \frac{1+w^{-1} w^{\prime-1}}{2}\left(z z^{\prime}\right)^{m-1}\left(w w^{\prime}\right)^{n} . \\
& \psi_{0, m n}=\frac{1}{\sqrt{S_{2}}} \int_{-\pi}^{\pi} \frac{d \theta}{2}\left(e^{-i m \theta-n \gamma(\theta)} \psi^{\dagger}(\theta)+e^{i m \theta+n \gamma(\theta)} \psi(\theta)\right) \\
& \quad=\frac{1}{\sqrt{S_{2}}} \int_{M} d U z^{m} w^{n} \psi(U) .
\end{aligned}
$$

Theorem 5.2.2. For $T<T_{c}$,

$$
\begin{aligned}
& \operatorname{Nr}\left(\bar{s}_{m n}\right)=\left\langle\bar{s}_{m n}\right\rangle e^{\bar{\rho}_{m n} / 2}, \\
& \left\langle\bar{s}_{m n}\right\rangle=\left(1-S_{1}^{-2} S_{2}^{-2}\right)^{1 / 8} \text {. } \\
& \bar{\rho}_{m n}=\iint_{-\pi}^{\pi} \underline{d \theta} \underline{d \theta^{\prime}}\left(\psi^{\dagger}(\theta) \psi(\theta)\right)\left(\begin{array}{ll}
\bar{R}_{m n}^{-}-\left(\theta, \theta^{\prime}\right) & \bar{R}_{m n}^{-+}\left(\theta, \theta^{\prime}\right) \\
\bar{R}_{m n}^{+-}\left(\theta, \theta^{\prime}\right) & \bar{R}_{m n}^{++}\left(\theta, \theta^{\prime}\right)
\end{array}\right)\left(\begin{array}{l}
\psi^{\dagger}\left(\theta^{\prime}\right) \\
\psi\left(\theta^{\prime}\right)
\end{array}\right) \\
& =\iint_{M \times M} d U d U^{\prime} \bar{R}_{m n}\left(U, U^{\prime}\right) \psi(U) \psi\left(U^{\prime}\right), \\
& \bar{R}_{m n}^{\sigma \sigma^{\prime}}\left(\theta, \theta^{\prime}\right)=\frac{2 \sinh \frac{\sigma \gamma(\theta)-\sigma^{\prime} \gamma\left(\theta^{\prime}\right)}{2}}{1-e^{-i\left(\sigma \theta+\sigma^{\prime} \theta^{\prime}-i 0\right)}} \\
& \times e^{i(m-1)\left(\sigma \theta+\sigma^{\prime} \theta^{\prime}\right)+n\left(\sigma \gamma(\theta)+\sigma^{\prime} \gamma\left(\theta^{\prime}\right)\right)} \quad\left(\sigma, \sigma^{\prime}= \pm\right),
\end{aligned}
$$




$$
R_{m n}\left(U, U^{\prime}\right)=\frac{w-w^{\prime}}{1-z^{-1} z^{\prime-1}}\left(z z^{\prime}\right)^{m-1}\left(w w^{\prime}\right)^{n-\frac{1}{2}} .
$$

For $T>T_{c}$ we may change $\rho_{m n}$ by $\rho_{m n}+\psi_{1, m n} \psi_{0, m n}$ for any $\psi_{1, m n} \in W$ (see Theorem 1.2.8). The particular choice

$$
\psi_{1, m n}=\int_{M} d U\left(\frac{S_{1}}{S_{2}} z^{m-1} w^{n}+z^{m} w^{n+1}\right) \psi(U)
$$

leads to the following.

Theorem 5.2.3. For $T>T_{c}$,

$$
\begin{aligned}
& \operatorname{Nr}\left(s_{m n}\right)=\left(1-S_{1}^{2} S_{2}^{2}\right)^{1 / 8} \psi_{0 . m n} e_{m n}^{\rho_{m n}^{\prime 2}} . \\
& \rho_{m n}^{\prime}=\iint_{-\pi}^{\pi} \underline{d \theta} \underline{d \theta^{\prime}}\left(\psi^{\dagger}(\theta) \psi(\theta)\right)\left(\begin{array}{cc}
R_{m n}^{\prime--}\left(\theta, \theta^{\prime}\right) & R_{m n}^{\prime-+}\left(\theta, \theta^{\prime}\right) \\
R_{m n}^{\prime+-}\left(\theta, \theta^{\prime}\right) & R_{m n}^{\prime++}\left(\theta, \theta^{\prime}\right)
\end{array}\right)\left(\begin{array}{c}
\psi^{\dagger}\left(\theta^{\prime}\right) \\
\psi\left(\theta^{\prime}\right)
\end{array}\right) \\
& =\iint_{M \times M} d U d U^{\prime} R_{m n}^{\prime}\left(U, U^{\prime}\right) \psi(U) \psi\left(U^{\prime}\right), \\
& R_{m n}^{\prime \sigma \sigma^{\prime}}\left(\theta, \theta^{\prime}\right)=\frac{2 \sinh \frac{\sigma \gamma(\theta)-\sigma^{\prime} \gamma\left(\theta^{\prime}\right)}{2}}{1-e^{-i\left(\sigma \theta+\sigma^{\prime} \theta^{\prime}-i 0\right)}} \\
& \quad \times e^{i m\left(\sigma \theta+\sigma^{\prime} \theta^{\prime}\right)+\left(n+\frac{1}{2}\right)\left(\sigma \gamma(\theta)+\sigma^{\prime} \gamma\left(\theta^{\prime}\right)\right)} \quad\left(\sigma, \sigma^{\prime}= \pm\right), \\
& R_{m n}^{\prime}\left(U, U^{\prime}\right)=\frac{w-w^{\prime}}{1-z^{-1} z^{\prime-1}}\left(z z^{\prime}\right)^{m}\left(w w^{\prime}\right)^{n} .
\end{aligned}
$$

Proof. We note that for $(z, w),\left(z^{\prime}, w^{\prime}\right) \in M^{c}$ we have

$$
S_{2} \frac{w-w^{\prime}}{1-z^{-1} z^{\prime-1}}+S_{1} \frac{z-z^{\prime}}{1-w^{-1} w^{\prime-1}}=0
$$

Without loss of generality we may assume that $m=1$ and $n=0$. Using (5.2.47) we have

$$
\begin{aligned}
& \rho_{10}+\psi_{1,10} \psi_{0,10} \\
&= \iint_{M \times M} d U d U^{\prime}\left\{\frac{w-w^{\prime}}{1-z^{-1} z^{\prime-1}} \cdot \frac{1+w^{-1} w^{\prime-1}}{2}\right. \\
&\left.\quad+\frac{S_{1}}{2 S_{2}}\left(z^{\prime}-z\right)+z z^{\prime}\left(w-w^{\prime}\right)\right\} \psi(U) \psi\left(U^{\prime}\right) \\
&= \iint_{M \times M} d U d U^{\prime}\left\{-\frac{S_{1}}{2 S_{2}} \cdot \frac{1+w^{-1} w^{\prime-1}}{1-w^{-1} w^{\prime-1}}\left(z-z^{\prime}\right)\right. \\
&\left.\quad+\frac{S_{1}}{2 S_{2}}\left(z^{\prime}-z\right)+z z^{\prime}\left(w-w^{\prime}\right)\right\} \psi(U) \psi\left(U^{\prime}\right) \\
&= \iint_{M \times M} d U d U^{\prime}\left\{\frac{w-w^{\prime}}{1-z^{-1} z^{\prime-1}}+z z^{\prime}\left(w-w^{\prime}\right)\right\} \psi(U) \psi\left(U^{\prime}\right) \\
&= \iint_{M \times M} d U d U^{\prime} \frac{w-w^{\prime}}{1-z^{-1} z^{\prime-1}} z z^{\prime} \psi(U) \psi\left(U^{\prime}\right) .
\end{aligned}
$$


Remark. By different choices of $\psi_{1, m n}$ the following kernels are also admissible as $R_{m n}^{\prime}\left(U, U^{\prime}\right)$.

$$
\begin{aligned}
& \frac{w-w^{\prime}}{1-z^{-1} z^{\prime-1}}\left(z z^{\prime}\right)^{m-1}\left(w w^{\prime}\right)^{n} . \\
& \frac{w-w^{\prime}}{1-z^{-1} z^{\prime-1}}\left(z z^{\prime}\right)^{m}\left(w w^{\prime}\right)^{n-1} . \\
& \frac{w-w^{\prime}}{1-z^{-1} z^{\prime-1}}\left(z z^{\prime}\right)^{m-1}\left(w w^{\prime}\right)^{n-1} .
\end{aligned}
$$

Finally we express the auxiliary operators $p_{m n}$ and $q_{m n}$ in terms of $\psi(U)$. For $T>T_{c}$

$$
\begin{aligned}
& p_{m n}=\sqrt{\left(C_{1}+1\right) S_{2}^{* / 2}} \int_{M} d U \sqrt{\left(1-\alpha_{1} z^{-1}\right)\left(1-\alpha_{2}^{-1} z^{-1}\right)} z^{m} w^{n} \psi(U), \\
& q_{m n}=\sqrt{\left(C_{1}+1\right) S_{2}^{*} / 2} \int_{M} d U \sqrt{\left(1-\alpha_{1} z\right)\left(1-\alpha_{2}^{-1} z\right)} z^{m} w^{n} \varepsilon(U) \psi(U),
\end{aligned}
$$

where $\varepsilon(U)= \pm 1$ for $U \in M_{ \pm}$.

For $T<T_{c}$

$$
\begin{aligned}
& p_{m n}=i \sqrt{\left(C_{1}+1\right) S_{2}^{*} /\left(2 \alpha_{2}\right)} \int_{M} d U \sqrt{\left(1-\alpha_{1} z^{-1}\right)\left(1-\alpha_{2} z\right)} z^{m-1} w^{n} \varepsilon(U) \psi(U), \\
& q_{m n}=-i \sqrt{\left(C_{1}+1\right)} \overline{S_{2}^{*} /\left(2 \alpha_{2}\right)} \int_{M} d U \sqrt{\left(1-\alpha_{1} z\right)\left(1-\alpha_{2} z^{-1}\right)} z^{m} w^{n} \psi(U)
\end{aligned}
$$

\section{§5.3. Correlation Functions}

In this section, applying the product formulas (Theorems 1.4.3 and 1.4.4) we derive infinite series expressions (cf. [9], [10], [11]) for $k$-point correlation functions directly from the norm representations of spin operators.

Let $C_{ \pm}, C_{ \pm}^{\prime}$ denote the 1-cycles on $M^{c}$ defined by

$$
\begin{aligned}
& C_{ \pm}=\left\{(z, w) \in M^{c}\left|z=e^{i \theta},\right| w \mid \gtrless 1, \theta \in \boldsymbol{R} / 2 \pi \mathbb{Z}\right\}, \\
& C_{ \pm}^{\prime}=\left\{(z, w) \in M^{\boldsymbol{c}}\left|w=e^{i \theta},\right| z \mid \gtrless 1, \theta \in \boldsymbol{R} / 2 \pi \mathbb{Z}\right\} .
\end{aligned}
$$

In Figures 5.3.1 and 5.3.2, we show their locations. 
Figure 5.3.1 $z$-plane

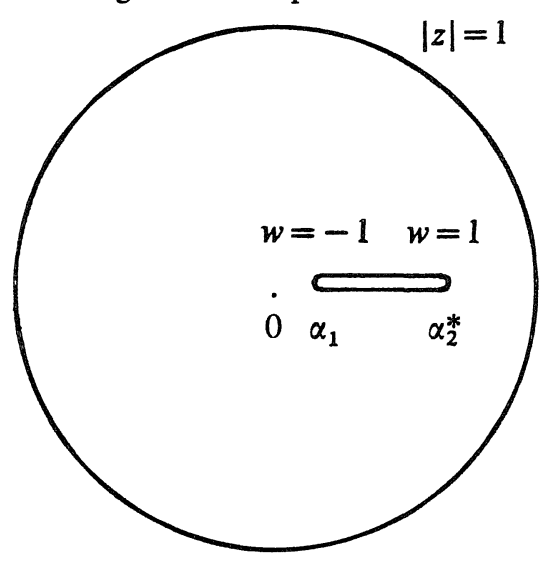

$$
\begin{gathered}
w=1 \quad w=-1 \\
\alpha_{2}^{*-1} \alpha_{1}^{-1} \\
\alpha_{2}^{*}= \begin{cases}\alpha_{2} & \left(T<T_{c}\right), \\
\alpha_{2}^{-1} & \left(T>T_{c}\right) .\end{cases}
\end{gathered}
$$

Figure 5.3.2 $M C$

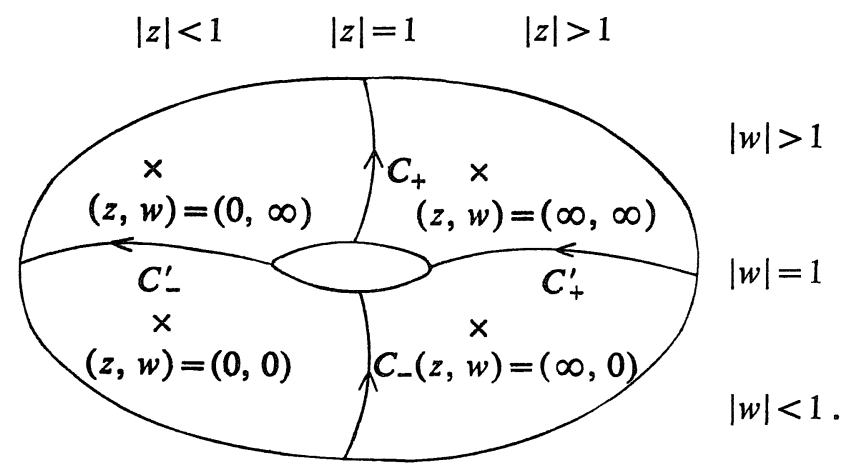

We define the $l$-form $\Omega_{l}$ on $\left(M^{c}\right)^{l}$ by

$$
\Omega_{l}=\left(\prod_{j=1}^{l} \frac{-w_{j}+w_{j+1}^{-1}}{1-z_{j}^{-1} z_{j+1}}\right) d U_{1} \wedge \cdots \wedge d U_{l},
$$

where we set $z_{l+1}=z_{1}$ and $w_{l+1}=w_{1}$. If we introduce another uniformizing parameter $\tilde{U}$ on $M^{\boldsymbol{C}}$ through

$$
d \tilde{U}=\frac{d w}{\pi i w\left(z-z^{-1}\right)}=-\frac{S_{1}}{S_{2}} d U
$$

$\Omega_{l}$ is rewritten as

$$
\Omega_{l}=\left(\prod_{j=1}^{l} \frac{-z_{j}+z_{j+1}^{-1}}{1-w_{j}^{-1} w_{j+1}}\right) d \widetilde{U}_{1} \wedge \cdots \wedge d \widetilde{U}_{l} .
$$

Here we have used (5.2.47) and the following. 


$$
S_{1} \frac{d z}{z\left(w-w^{-1}\right)}+S_{2} \frac{d w}{w\left(z-z^{-1}\right)}=0 .
$$

$\Omega_{l}$ is holomorphic except for simple poles at

$$
\Delta_{l}^{(j)}=\left\{\left(U_{1}, \ldots, U_{l}\right) \in\left(M^{C}\right)^{l} \mid U_{j}=U_{j+1}\right\},
$$

$(j=1, \ldots, l)$ where $U_{l+1}=U_{1}$. The residue at $\Delta_{l}^{(j)}$ is given by

$$
\operatorname{res}_{\Delta_{i}^{(j)}} \Omega_{l}=(-)^{j} \Omega_{l-1} / \pi i,
$$

where we identify $\Delta_{l}^{(j)}$ with $\left(M^{c}\right)^{l-1}$ (*) $^{(*)}$

Let $\left(m_{1}, n_{1}\right), \ldots,\left(m_{k}, n_{k}\right)$ be $k$ distinct lattice points. We choose permutations $\sigma$ and $\tau$ so that $m_{\sigma(1)} \leqq \cdots \leqq m_{\sigma(k)}$ and $n_{\tau(1)} \leqq \cdots \leqq n_{\tau(k)}$, respectively. We denote by $\underset{m}{<}$ the ordering by $\sigma$, namely for $1 \leqq v, v^{\prime} \leqq k$

$$
v \underset{m}{\nu^{\prime}} \Leftrightarrow \sigma^{-1}(v)<\sigma^{-1}\left(v^{\prime}\right) \text {. }
$$

Likewise $<_{n}$ is defined. We set

$$
m_{v v^{\prime}}=m_{v}-m_{v^{\prime}}, \quad n_{v v^{\prime}}=n_{v}-n_{v^{\prime}},
$$

and denote by $C_{v v^{\prime}}$, the 1-cycle defined by

$$
C_{v v^{\prime}}=\left\{\begin{array}{lll}
C_{+} & \text {if } & v>v^{\prime}, \\
0 & \text { if } & v=v^{\prime}, \\
C_{-} & \text {if } & v<v^{\prime}
\end{array}\right.
$$

We also set

$$
C_{v v^{\prime}}^{\prime}=\left\{\begin{array}{lll}
C_{+}^{\prime} & \text { if } & v>v^{\prime}, \\
0 & \text { if } & v=v^{\prime}, \\
C_{-}^{\prime} & \text { if } & v>v_{m}^{\prime} .
\end{array}\right.
$$

First we assume that $T<T_{c}$. From (1.4.12), (5.2.43) and (5.2.44) we have the following ([9]).

Theorem 5.3.1. For $T<T_{c}$,

$$
\rho_{k}\left(\left(m_{1}, n_{1}\right), \ldots,\left(m_{k}, n_{k}\right)\right)=\left(1-S_{1}^{-2} S_{2}^{-2}\right)^{\frac{k}{8}} \exp \left(-\sum_{l=2}^{\infty} \frac{F_{k}^{(l)}}{2 l}\right)
$$

where $F_{k}^{(l)}=\sum_{v_{1}, \ldots, v_{l}=1}^{k} F_{k v_{1}, \ldots, v_{l}}^{(l)}$,

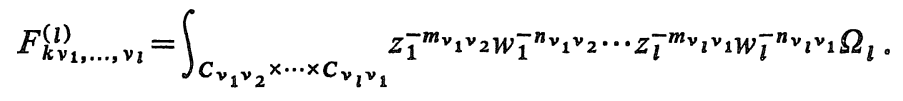

(*) For a closed form $\omega$ with a simple pole at $\Delta=\{f=0\}$, the residue res ${ }_{d} \omega$ is defined to be $\omega /\left.d \log f\right|_{d}=\left.\theta\right|_{\triangle}$ where $\omega=d \log f_{\wedge} \theta+\varphi(\theta, \varphi$ : holomorphic). 
In (5.3.10) if $C_{v_{j-1} v_{j}}=C_{v_{\jmath} v_{j+1}}$ for some $j$, w'e deform these cycles so that

$$
\left|z_{j-1}\right|>\left|z_{j}\right| \text {. }
$$

Let $D_{4}$ denote the dihedral group of order 8, i.e. $D_{4}$ has two generators $A_{1}$ and $A_{2}$ satisfying $A_{1}^{2}=A_{2}^{2}=\left(A_{2} A_{1}\right)^{4}=1$.

By the definition the correlation function satisfies the following invariance with respect to $D_{4}$.

$$
\begin{aligned}
& \rho_{k}\left(\left(m_{1}, n_{1}\right), \ldots,\left(m_{k}, n_{k}\right) ; K_{1}, K_{2}\right) \\
& \quad=\rho_{k}\left(\left(m_{1},-n_{1}\right), \ldots,\left(m_{k},-n_{k}\right) ; K_{1}, K_{2}\right), \\
& \rho_{k}\left(\left(m_{1}, n_{1}\right), \ldots,\left(m_{k}, n_{k}\right) ; K_{1}, K_{2}\right) \\
& \quad=\rho_{k}\left(\left(n_{1}, m_{1}\right), \ldots,\left(n_{k}, m_{k}\right) ; K_{2}, K_{1}\right) .
\end{aligned}
$$

For the infinite series (5.3.9), (5.3.12) ${ }_{A_{1}}$ is easily checked using the invariance of $\Omega_{l}$ under the automorphism $(z, w) \mapsto\left(z, w^{-1}\right)$ of $M^{c}$. To check (5.3.12) $)_{A_{2}}$ is equivalent to show that

$$
\rho_{k}\left(\left(m_{1}, n_{1}\right), \ldots,\left(m_{k}, n_{k}\right)\right)=\left(1-S_{1}^{-2} S_{2}^{-2}\right)^{\frac{k}{8}} \exp \left(-\sum_{l=2}^{\infty} \frac{F_{k}^{\prime(l)}}{2 l}\right),
$$

where $F_{k}^{\prime(l)}=\sum_{v_{1}, \ldots, v_{l}=1}^{k} F_{k v_{1}, \ldots, v_{l}}^{\prime(l)}$,

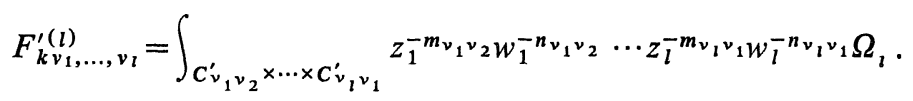

Here we used (5.3.4). In (5.3.10)' if $C_{v_{j-1} v_{j}}^{\prime}=C_{v_{j} v_{j+1}}^{\prime}$ for some $j$, we deform these cycles so that

$$
\left|w_{j-1}\right|>\left|w_{j}\right| \text {. }
$$

As mentioned in [9] $F_{k}^{(l)}$ is not equal to $F_{k}^{(l)}$ in general. When we deform the cycles from $C_{ \pm}$into $C_{ \pm}^{\prime}$ in order to obtain $F_{k, v_{1}, \ldots, v_{l}}^{\prime(l)}$ from $F_{k v_{1}, \ldots, v_{l}}^{(l)}$, residual terms arise from (5.3.6). We shall give a sketch of the direct proof of the cancellation in the whole sum $\sum_{l=2}^{\infty} F_{k}^{\prime(l)} / 2 l$.

A residual term in $F_{k v_{1}, \ldots, v_{l}}^{(l)}$ appears from $\Delta_{l}^{(j)}$ in the following six cases.

Case 1. $v_{j}<v_{j+1}<_{n} v_{j+2}$ and $v_{j}, v_{j+2} \underset{m}{<} v_{j+1}$.

Case 2. $v_{j}>v_{j+1}>v_{j+2}$ and $v_{j}, v_{j+2} \underset{m}{<} v_{j+1}$.

Case 3. $v_{j}<v_{j+1}<v_{j+2}$ and $v_{j}<_{m} v_{j+1}<_{m} v_{j+2}$.

Case 4. $\quad v_{j}>v_{j+1}>v_{j+2}$ and $v_{j}>v_{j+1}>v_{j+2}$.

Case 5. $v_{j}, v_{j+2} \underset{n}{<j+1}$ and $v_{j}<_{m} v_{j+1} \underset{m}{<} v_{j+2}$. 
Case 6. $v_{j}, v_{j+2} \underset{n}{<v_{j+1}}$ and $v_{j} \underset{m}{>} v_{j+1} \underset{m}{>} v_{j+2}$.

In Case 1, at first $C_{v_{j} v_{j+1}}$ is located to the right (in Figure 5.3.2) of $C_{v_{j+1} v_{j+2}}$ because of the condition (5.3.12). Since $C_{v_{j} v_{j+1}}$ (resp. $C_{v_{j+1} v_{j+2}}$ ) is deformed into $C_{-}^{\prime}$ (resp. $C_{+}^{\prime}$ ) in this case, we must reverse their positions. Thus we get $-2 F_{k v_{1} \cdots v_{j} v_{j}+2 \cdots v_{l}}^{(l-1)}$ as the residue. Likewise we need to reverse the order of cycles in the above six cases.

After the reversing corresponding to Cases 1 and 2, the sum $\sum_{l=2}^{\infty} F_{k}^{(l)} / 2 l$ changes to $\sum_{l=2}^{\infty}{ }^{1} F_{k}^{(l)} / 2 l$ where

$$
\begin{aligned}
& { }^{1} F_{k}^{(l)}=\sum_{v_{1}, \ldots, v_{l}=1}^{k} \varepsilon_{1}\left(v_{1}, \ldots, v_{l}\right)^{1} F_{v_{1}, \ldots, v_{l}}^{(l)}, \\
& \varepsilon_{1}\left(v_{1}, \ldots, v_{l}\right)=(-)^{\ddagger_{1}\left(v_{1}, \ldots, v_{l}\right),} \\
& \#_{1}\left(v_{1}, \ldots, v_{l}\right)=\text { the cardinal number of the set } \\
& \quad\left\{v \mid v_{j}<v<v_{n+1}, v_{j}, v_{j+1} \underset{m}{<} \quad \text { for some } j\right\} \\
& \cup\left\{v \mid v_{j}>v>v_{n} v_{j+1}, v_{j}, v_{j+1}<_{m} v \quad \text { for some } j\right\} .
\end{aligned}
$$

Here ${ }^{1} F_{k v_{1}, \ldots, v_{l}}^{(l)}$ is given by (5.3.10) with the following prescription for a pair satisfying $C_{v_{j-1} v_{j}}=C_{v_{j} v_{j+1}}$.

$$
\begin{aligned}
& \text { (5.3.11) })_{1} \quad\left|z_{j-1}\right|<\left|z_{j}\right| \text { if } C_{v_{j-1} v_{j}}^{\prime}=C_{-}^{\prime} \text { and } C_{v_{j} v_{j+1}}^{\prime}=C_{+}^{\prime} \text {, } \\
& \left|z_{j-1}\right|>\left|z_{j}\right| \quad \text { otherwise. }
\end{aligned}
$$

Next we perform the reversing for the Cases 3 and 4 . The result is $\sum_{l=2}^{\infty}{ }^{2} F_{k}^{(l)} / 2 l$ where

$$
\begin{aligned}
& { }^{2} F_{k}^{(l)}=\sum_{v_{1}, \ldots, v_{l}=1}^{k} \varepsilon_{1}\left(v_{1}, \ldots, v_{l}\right) \varepsilon_{2}\left(v_{1}, \ldots, v_{l}\right)^{2} F_{k v_{1}, \ldots, v_{l}}^{(l)}, \\
& \varepsilon_{2}\left(v_{1}, \ldots, v_{l}\right)=(-)^{\#_{2}\left(v_{1}, \ldots v_{l}\right)} \text {, } \\
& \#_{2}\left(v_{1}, \ldots, v_{l}\right)=\text { the cardinal number of the set } \\
& \left\{v \mid v_{j}<_{n} v<_{n} v_{j+1}, v_{j}<_{m} v<_{m} v_{j+1} \quad \text { for some } j\right\} \\
& \cup\left\{v \mid v_{j}>_{n} v>_{n} v_{j+1}, \quad v_{j}>_{m} v>_{m} v_{j+1} \quad \text { for some } j\right\} \text {. }
\end{aligned}
$$

Here ${ }^{2} F_{k v_{1}, \ldots, v_{l}}^{(l)}$ is given by (5.3.10) with the following prescription for a pair satisfying $C_{v_{j-1} v_{j}}=C_{v_{j} v_{j+1}}$.

$$
\begin{array}{lll}
\left|z_{j-1}\right|<\left|z_{j}\right| & \text { if } \quad C_{v_{j-1} v_{j}}^{\prime}=C_{-}, \\
\left|z_{j-1}\right|>\left|z_{j}\right| & \text { if } \quad C_{v_{j-1} v_{j}}^{\prime}=C_{+} .
\end{array}
$$

Now we deform $C_{ \pm}$into $C_{ \pm}^{\prime}$ and obtain $\sum_{l=2}^{\infty}{ }^{3} F_{k}^{(l)} / 2 l$ where 


$$
\begin{aligned}
& { }^{3} F_{k}^{(l)}=\sum_{v_{1}, \ldots, v_{l}=1}^{k} \varepsilon_{1}\left(v_{1}, \ldots, v_{l}\right) \varepsilon_{2}\left(v_{1}, \ldots, v_{l}\right) \varepsilon_{3}\left(v_{1}, \ldots, v_{l}\right) \\
& \times{ }^{3} F_{k v_{1}, \ldots, v_{l}}^{(l)},
\end{aligned}
$$

Here ${ }^{3} F_{k v_{1}, \ldots, v_{k}}^{(l)}$ is given by (5.3.10) $)^{\prime}$ with the following prescription for a pair satisfying $C_{v_{j-1} v_{j}}^{\prime}=C_{v_{j} v_{j+1}}^{\prime}$.

$$
\begin{aligned}
& \left|w_{j-1}\right|<\left|w_{j}\right| \text { if } C_{v_{j-1} v_{j}}=C_{-} \text {and } C_{v_{j} v_{j+1}}=C_{+}, \\
& \left|w_{j-1}\right|>\left|w_{j}\right| \text { otherwise. }
\end{aligned}
$$

We can show that

$$
\begin{aligned}
& \varepsilon_{1}\left(v_{1}, \ldots, v_{l}\right) \varepsilon_{2}\left(v_{1}, \ldots, v_{l}\right) \varepsilon_{3}\left(v_{1}, \ldots, v_{l}\right)=(-)^{\# 1}\left(v_{1}, \ldots, v_{l}\right) \\
& \#_{1}^{\prime}\left(v_{1}, \ldots, v_{l}\right)=\text { the cardinal number of the set } \\
& \left\{v \mid v_{j}<_{m}<_{m} v_{j+1}, v_{j}, v_{j+1}<_{n} v \quad \text { for some } j\right\} \\
& U\left\{v \mid v_{j}>_{m} v>_{m} v_{j+1}, v_{j}, v_{j+1} \underset{n}{<v} \text { for some } j\right\} .
\end{aligned}
$$

Hence after reversing cycles for the Cases 5 and 6, we obtain the desired sum $\sum_{l=2}^{\infty} F_{k}^{\prime(l)}$.

If $\left|n_{v}-n_{v^{\prime}}\right| \gg 1$ for any pair $\left(v, v^{\prime}\right)$, the convergence of the sum $\sum_{l=2}^{\infty} F_{k}^{(l)} / 2 l$ is obvious by the same argument as in Proposition 4.5. Indeed $\left|w_{j}\right|^{-n_{v_{j} v_{j+1}}}$ is much smaller than 1 on $C_{v_{j} v_{j+1}}$ and serves as a damping factor. Now we shall show that (5.3.9) is convergent if for any pair $\left(v, v^{\prime}\right)$ either $\left|n_{v}-n_{v^{\prime}}\right| \gg 1$ or $\left|m_{v}-m_{v^{\prime}}\right| \gg 1$. In fact, if $\left|m_{v}-m_{v^{\prime}}\right| \gg 1$, we deform $C_{v v^{\prime}}$ into $C_{v v^{\prime}}^{\prime}$ Then $|z|^{-m_{v v^{\prime}}}$ is much smaller than 1 on $C_{v^{\prime}{ }^{\prime}}^{\prime}$. Of course we should estimate the residual terms. Let

$$
\sum_{s=0}^{l} \sum_{\mu_{1}, \ldots, \mu_{s}=1}^{k} c_{\mu_{1}, \ldots, \mu_{s}}^{\prime} F_{k \mu_{1}, \ldots, \mu_{s}}^{\prime(s)}+\sum_{s=0}^{l} \sum_{\mu_{1}, \ldots, \mu_{s}=1}^{k} c_{\mu_{1}, \ldots, \mu_{s}} F_{k \mu_{1}, \ldots, \mu_{s}}^{(s)}
$$

be the terms obtained from $F_{k v_{1}, \ldots, v_{l}}^{(l)}$. Then it is easy to see the following conditions, which are sufficient for the convergence proof.

$$
\left|c_{\mu_{1}, \ldots, \mu_{s}}^{\prime}\right|<2^{l-s}, \quad\left|c_{\mu_{1}, \ldots, \mu_{s}}\right|<2^{l-s} .
$$

The cardinal numbers of the sets

$$
\left\{\left(\mu_{1}, \ldots, \mu_{s}\right) \mid c_{\mu_{1}, \ldots, \mu_{s}}^{\prime} \neq 0\right\}, \quad\left\{\left(\mu_{1}, \ldots, \mu_{s}\right) \mid c_{\mu_{1}, \ldots, \mu_{s}} \neq 0\right\}
$$

are less than $2^{l}$ for a sufficiently large $l$. Hence 


$$
c_{\mu_{1}, \ldots, \mu_{s}}^{\prime}=c_{\mu_{1}, \ldots, \mu_{s}}=0 \quad \text { if } \quad s<\left[\frac{l}{k}\right]
$$

for a sufficiently large $l$.

Now we consider the case $T>T_{c^{*}}$. We set $l$-form $\widehat{\Omega}_{l}$ on $\left(M^{c}\right)^{l}$.

$$
\begin{aligned}
\hat{\Omega}_{l} & =\frac{-1}{S_{2}}\left(\prod_{j=1}^{l-1} \frac{-w_{j}+w_{j+1}^{-1}}{1-z_{j}^{-1} z_{j+1}}\right) d U_{1} \wedge \cdots \wedge d U_{l} \\
& =\frac{1}{S_{1}}\left(\prod_{j=1}^{l-1} \frac{-z_{j}+z_{j+1}^{-1}}{1-w_{j}^{-1} w_{j+1}}\right) d \widetilde{U}_{1} \wedge \cdots \wedge d \widetilde{U}_{l} .
\end{aligned}
$$

$\hat{\Omega}_{l}$ is holomorphic except for simple poles at $\Delta_{l}^{(j)}(j=1, \ldots, l-1)$ and $\left(z_{l}, w_{l}\right)=$ $(\infty, \infty)$ and $\left(z_{l}, w_{l}\right)=(0,0)$. The residues are as follows.

$$
\begin{aligned}
& \operatorname{res}_{\Delta l}^{(j)} \hat{\Omega}_{l}=(-)^{j} \hat{\Omega}_{l-1} / \pi i . \\
& \operatorname{res}_{\substack{z_{l}=\infty \\
w_{l}=\infty}} \hat{\Omega}_{l}=\hat{\Omega}_{l-1} / \pi i . \\
& \operatorname{res}_{z_{z_{l}=0}=0} \hat{\Omega}_{l}=(-)^{l} \hat{\Omega}_{l-1} / \pi i .
\end{aligned}
$$

From (1.4.12) and Theorem 5.2.3, we have the following ([9]).

Theorem 5.3.2. For $T>T_{c}$,

$$
\begin{aligned}
& \rho_{k}\left(\left(m_{1}, n_{1}\right), \ldots,\left(m_{k}, n_{k}\right)\right) \\
& \quad=\left(1-S_{1}^{2} S_{2}^{2}\right)^{\frac{k}{8}} \cdot \operatorname{Pfaffian} G_{k} \cdot \exp \left(-\sum_{l=2}^{\infty} \frac{F_{k}^{(l)}}{2 l}\right)
\end{aligned}
$$

where $F_{k}^{(l)}$ is the same as in Theorem 5.2.1, and $G_{k}=\sum_{l=1}^{\infty} G_{k}^{(l)}$ is a skew-symmetric $k \times k$ matrix given by

$$
G_{k v v^{\prime}}^{(l)}=\sum_{v_{1}, \ldots, v_{l-1}=1}^{k} G_{k \sigma^{-1}(v) v_{1} \ldots v_{l-1} \sigma^{-1}\left(v^{\prime}\right)}^{(l)}
$$

where

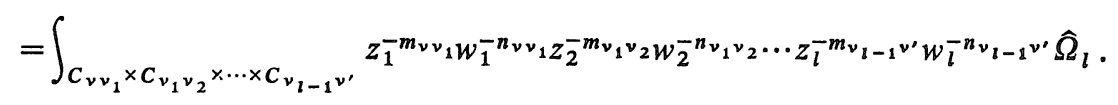

The expression (5.3.19) is derived from (5.2.46). We may adopt any one of $(5.2 .46)_{1} \sim(5.2 .46)_{3}$. Then the following are substituted for $G_{k v v_{1} \ldots v_{l}-1}^{(l)} v^{\prime}$. $(5.3 .19)_{1}$

$$
\begin{aligned}
& { }^{1} G_{k v v_{1} \ldots v_{l-1} v^{\prime}}^{(l)}
\end{aligned}
$$

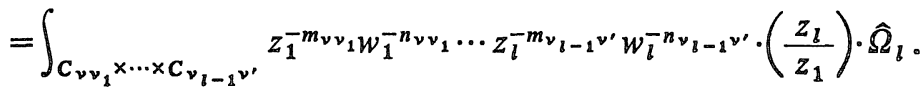

This is the choice of [9]. 


$$
\begin{aligned}
& { }^{2} G_{k v v_{1} \cdots v_{l-1} v^{\prime}}^{(l)}
\end{aligned}
$$

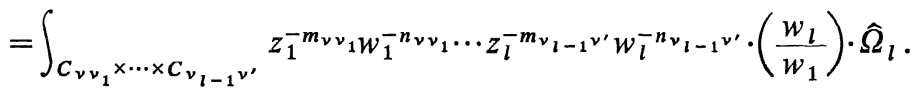

$$
\begin{aligned}
& { }^{3} G_{k v v_{1} \cdots v_{l-1} v^{\prime}}^{(l)}
\end{aligned}
$$

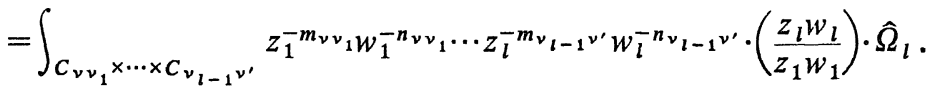

$G_{k v v_{1} \ldots v_{l-1} v^{\prime}}^{(l)}$ is transformed into $-{ }^{2} G_{k v v_{1} \ldots v_{l-1} v^{\prime}}^{(l)}$ under the action of $A_{1}$.

$$
\begin{aligned}
& G_{k v v_{1} \cdots v_{l-1} v^{\prime}}^{(l)}\left(\left(m_{1},-n_{1}\right), \ldots,\left(m_{k},-n_{k}\right) ; K_{1}, K_{2}\right) \\
= & -{ }^{2} G_{k v v_{1} \cdots v_{l-1} v^{\prime}}^{(l)}\left(\left(m_{1}, n_{1}\right), \ldots,\left(m_{k}, n_{k}\right) ; K_{1}, K_{2}\right) .
\end{aligned}
$$

Since the $n$-ordering is entirely reversed by $A_{1},(5.3 .20)$ implies the invariance of $\rho_{k}$.

In order to show the invariance of $\rho_{k}$ under the action of $A_{2}$, we must prove by deforming cycles from $C_{ \pm}$into $C_{ \pm}^{\prime}$ that

$$
\text { Pfaffian } G_{k}=\text { Pfaffian } G_{k}^{\prime}
$$

where $G_{k}^{\prime}=\sum_{l=1}^{\infty} G_{k}^{(l)}$ is given by

$$
\begin{aligned}
& G_{k \nu \nu^{\prime}}^{\prime(l)}=\sum_{v_{1}, \ldots, v_{l-1}=1}^{k} G_{k \tau^{-1}(v) v_{1} \ldots v_{l-1} \tau^{-1}\left(v^{\prime}\right)}^{(l)}, \\
& G_{k \nu v_{1} \cdots v_{l-1} v^{\prime}}^{(l)}
\end{aligned}
$$

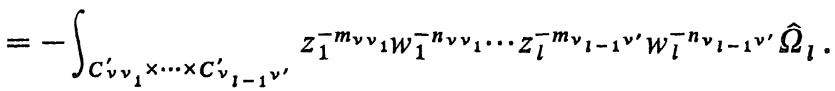

By the same argument as for $F_{k}^{(l)}$ we can show that $G_{k v v^{\prime}}$ is equal to $-\varepsilon\left(v, v^{\prime}\right) \cdot G_{k v v^{\prime}}^{\prime}$ where

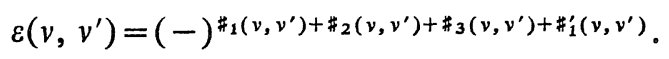

It is also easy to see that for any partition

$$
\begin{aligned}
& \left\{v_{1}, v_{2}\right\} \cup\left\{v_{3}, v_{4}\right\} \cup \cdots \cup\left\{v_{k-1}, v_{k}\right\} \text { of }\{1, \ldots, k\} \\
& (-)^{\frac{k}{2}} \varepsilon\left(v_{1}, v_{2}\right) \cdots \varepsilon\left(v_{k-1}, v_{k}\right)=\operatorname{sgn} \sigma \cdot \operatorname{sgn} \tau .
\end{aligned}
$$

Hence (5.3.21) is valid.

The convergence of $G_{k}$ is similarly shown as for (5.3.9).

\section{§5.4. The Symplectic Model}

Let us now proceed to the construction of a lattice model which constitutes the symplectic counterpart of the Ising model. 
This time we start with a rectangular lattice $L$ of size $M \times N$ with cyclic boundary, on which a continuous variable $x_{m n} \in \boldsymbol{R}$ is attached to each site $(m, n)$ $(0 \leqq m \leqq M-1,0 \leqq n \leqq N-1)$. The total energy is given by

$$
E(x)=\sum_{m=0}^{M-1} \sum_{n=0}^{N-1}\left(C_{1} C_{2} x_{m n}^{2}-S_{1} x_{m n} x_{m+1 n}-S_{2} x_{m n} x_{m n+1}\right),
$$

where $C_{i}, S_{i}$ denote those in (5.1.40). The grand partition function is defined by the integral

$$
\begin{aligned}
& Z_{M N}=\int_{R^{M N}} d x e^{-E(x)} \\
& d x=\prod_{m=0}^{M-1} \prod_{n=0}^{N-1} d x_{m n} .
\end{aligned}
$$

Let $\Gamma$ be a finite union of open polygons on the dual lattice $L^{*}$, and regard it as a simplicial 1-chain with coefficients in $Z_{2}$ (Fig. 5.4.1). Given a $\Gamma$, the signature of a bond $b$ on $L$ is defined to be -1 if $\Gamma$ crosses $b$, and to be 1 otherwise. We denote by $\varepsilon_{m n}^{(1)}(\Gamma)$ (resp. $\varepsilon_{m n}^{(2)}(\Gamma)$ ) the signature of the horizontal (resp. vertical) bond joining $(m, n)$ and $(m+1, n)$ (resp. $(m, n)$ and $(m, n+1))$.

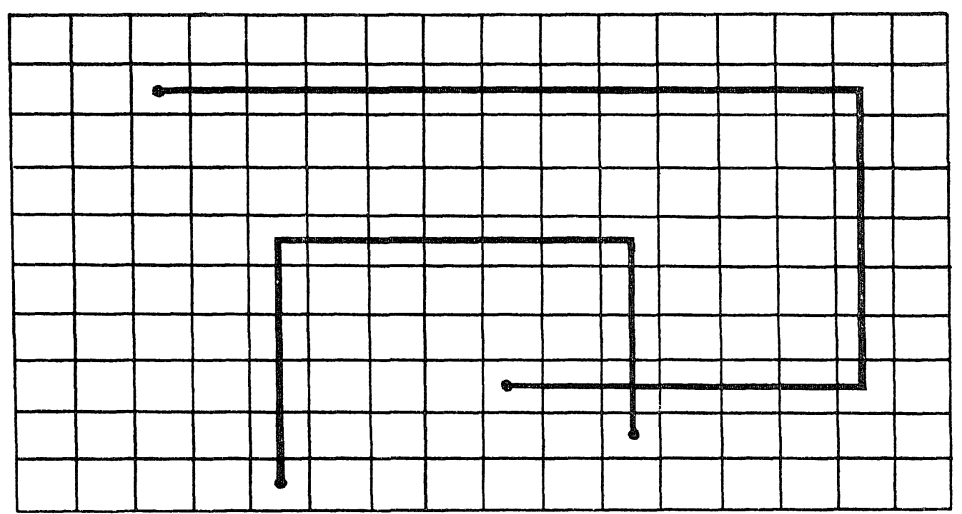

Figure 5.4.1

In the sequel we shall deal with only those $\Gamma$ 's which lie entirely in the interior of $L$. In this case the homology class of $\Gamma$ depends only on the boundary $\partial \Gamma$ (=the set of endpoints of $\Gamma$ ). Now set

$$
\begin{array}{r}
E_{\Gamma}(x)=\sum_{m=0}^{M-1} \sum_{n=0}^{N-1}\left(C_{1} C_{2} x_{m n}^{2}-S_{1} \varepsilon_{m n}^{(1)}(\Gamma) x_{m n} x_{m+1 n}\right. \\
\left.-S_{2} \varepsilon_{m n}^{(2)}(\Gamma) x_{m n} x_{m n+1}\right)
\end{array}
$$

and define an analogue of the correlation function (5.1.3) by 


$$
\rho(\partial \Gamma)=Z_{M N}^{-1} \int_{R^{M N}} d x e^{-E_{\Gamma}(x)} .
$$

The notation $\rho(\partial \Gamma)$ is justified by the following:

Proposition 5.4.1 The definition (5.4.4) depends only on $\partial \Gamma$.

Proof. Denote by $\rho_{\Gamma}$ the right member of (5.4.4). We are to prove that, if two chains $\Gamma, \Gamma^{\prime}$ are homologous, then $\rho_{\Gamma}=\rho_{\Gamma^{\prime}}$. It suffices to consider the case $\Gamma^{\prime}=\Gamma+\partial \square$, where $\square$ denotes a minimal square on the dual lattice $L^{*}$ centered at some point $\left(m_{0}, n_{0}\right) \in L$. Set

$$
x_{m n}^{\prime}=\left\{\begin{array}{cl}
x_{m n} & \left((m, n) \neq\left(m_{0}, n_{0}\right)\right) \\
-x_{m_{0} n_{0}} & \left((m, n)=\left(m_{0}, n_{0}\right)\right) .
\end{array}\right.
$$

Then it is easy to verify that $E_{\Gamma^{\prime}}(x)=E_{\Gamma}\left(x^{\prime}\right)$. Therefore the change of integration variable (5.4.5) proves our assertion.

Calculation of the partition function (5.4.2) is straightforward. More generally we consider the generating function

$$
\begin{aligned}
& Z_{M N}[J]=\int_{R^{M N}} d x e^{J \cdot x} e^{-E(x)} \\
& J=\left(J_{m n}\right)_{\substack{0 \leqq m \leqq M-1 \\
0 \leqq n \leqq N-1}}, \quad J \cdot x=\sum_{m=0}^{M-1} \sum_{n=0}^{N-1} J_{m n} x_{m n} .
\end{aligned}
$$

In terms of the Fourier transformation

$$
\begin{array}{r}
\hat{x}_{\mu \nu}=\sum_{m=0}^{M-1} \sum_{n=0}^{N-1} e^{-i m \theta_{\mu}-i n \theta_{\nu}^{\prime}} x_{m n} \\
\hat{J}_{\mu \nu}=\sum_{m=0}^{M-1} \sum_{n=0}^{N-1} e^{-i m \theta_{\mu}-i n \theta_{\nu}^{\prime}} J_{m n} \\
\left(\theta_{\mu}=\frac{2 \pi \mu}{M}, \theta_{v}^{\prime}=\frac{2 \pi v}{N} ; \mu=0,1, \ldots, M-1 \bmod M\right. \\
v=0,1, \ldots, N-1 \bmod N)
\end{array}
$$

we have

$$
\begin{aligned}
& E(x)=\frac{1}{M N} \sum_{\mu=0}^{M-1} \sum_{v=0}^{N-1} \Delta\left(\theta_{\mu}, \theta_{v}^{\prime}\right) \hat{x}_{\mu v} \hat{x}_{-\mu,-v} \\
& J \cdot x=\frac{1}{2 M N} \sum_{\mu=0}^{M-1} \sum_{v=0}^{N-1}\left(\hat{J}_{\mu v} \hat{x}_{-\mu,-v}+\hat{J}_{-\mu,-v} \hat{x}_{\mu v}\right)
\end{aligned}
$$

with $\Delta\left(\theta_{\mu}, \theta_{v}^{\prime}\right)=C_{1} C_{2}-S_{1} \cos \theta_{\mu}-S_{2} \cos \theta_{v}^{\prime}>0$. Since $\left(x_{m n}\right) \mapsto \sqrt{M N}^{-1}\left(\hat{x}_{\mu v}\right)$ is a unitary transformation, (5.4.8) shows that the eigenvalues of the quadratic form $E(x)$ are $\Delta\left(\theta_{\mu}, \theta_{v}^{\prime}\right)(0 \leqq \mu \leqq M-1,0 \leqq v \leqq N-1)$. Making use of the formula 


$$
\begin{aligned}
& \int \cdots \int_{\boldsymbol{R}^{N}} d x_{1} \cdots d x_{N} e^{-t x A x}=\sqrt{\pi^{N}}(\operatorname{det} A)^{-1 / 2} \\
& \quad\left({ }^{t} x=\left(x_{1}, \ldots, x_{N}\right), A={ }^{t} A, \operatorname{Re} A . \text { is positive definite }\right)
\end{aligned}
$$

we obtain the following.

$$
\begin{aligned}
& Z_{M N}=\pi^{M N / 2}\left(\prod_{\mu=0}^{M-1} \prod_{v=0}^{N-1} \Delta\left(\theta_{\mu}, \theta_{v}^{\prime}\right)\right)^{-1 / 2} \\
& Z_{M N}[J]=Z_{M N} \exp \left(\frac{1}{2} \sum_{m n} \sum_{m^{\prime} n^{\prime}} \frac{1}{2} a_{m-m^{\prime}, n-n^{\prime}} J_{m n} J_{m^{\prime} n^{\prime}}\right) \\
& a_{m n}=\frac{1}{M N} \sum_{\mu=0}^{M-1} \sum_{v=0}^{N-1} \frac{1}{\Delta\left(\theta_{\mu}, \theta_{v}^{\prime}\right)} e^{-i m \theta_{\mu}-i n \theta_{v}^{\prime}} .
\end{aligned}
$$

Here we have used $\Delta\left(\theta, \theta^{\prime}\right)=\Delta\left(-\theta, \theta^{\prime}\right)=\Delta\left(\theta,-\theta^{\prime}\right)$. In particular (5.4.6) and (5.4.11) imply

$$
Z_{M N}^{-1} \int_{\mathbb{R}^{M N}} d x x_{m n} x_{m^{\prime} n^{\prime}} e^{-E(x)}=\frac{1}{2} a_{m-m^{\prime}, n-n^{\prime}}
$$

In order to obtain the "correlation functions" $\rho(\partial \Gamma)$, we use the transfer matrix formalism. In the sequel we identify an integral operator on $\mathbb{R}^{M} F$ : $f(x) \mapsto \int_{\boldsymbol{R}^{M}} d x^{\prime} F\left(x, x^{\prime}\right) f\left(x^{\prime}\right)\left(x=\left(x_{0}, x_{1}, \ldots, x_{M-1}\right) \in \boldsymbol{R}^{M}, d x=d x_{0} d x_{1} \cdots d x_{M-1}\right)$ with the kernel function $F\left(x, x^{\prime}\right)$. Let $V_{1}, V_{2}$ be given by

$$
\begin{aligned}
& V_{1}\left(x, x^{\prime}\right)=\exp \left(-\sum_{m=0}^{M-1}\left(C_{1} C_{2} x_{m}^{2}-S_{1} x_{m} x_{m+1}\right)\right) \delta^{M}\left(x-x^{\prime}\right) \\
& V_{2}\left(x, x^{\prime}\right)=\exp \left(\sum_{m=0}^{M-1} S_{2} x_{m} x_{m}^{\prime}\right) \\
& \quad\left(\delta^{M}\left(x-x^{\prime}\right)=\delta\left(x_{0}-x_{0}^{\prime}\right) \delta\left(x_{1}-x_{1}^{\prime}\right) \cdots \delta\left(x_{M-1}-x_{M-1}^{\prime}\right)\right),
\end{aligned}
$$

and let $V=V_{1} V_{2}$. We have then

$$
\begin{aligned}
& V\left(x, x^{\prime}\right)=\exp \left(-\sum_{m=0}^{M-1}\left(C_{1} C_{2} x_{m}^{2}-S_{1} x_{m} x_{m+1}-S_{2} x_{m} x_{m}^{\prime}\right)\right) \\
& Z_{M N}=\int d x^{(0)} \cdots \int d x^{(N-1)} V\left(x^{(0)}, x^{(1)}\right) V\left(x^{(1)}, x^{(2)}\right) \cdots V\left(x^{(N-1)}, x^{(0)}\right) \\
& \quad=\operatorname{trace} V^{N}
\end{aligned}
$$

where we have set trace $F=\int_{R^{M}} d x F(x, x)$. We introduce also "free bose fields" $\phi_{m}, \pi_{m}(0 \leqq m \leqq M-1)$ through

$$
\begin{aligned}
& \phi_{m}\left(x, x^{\prime}\right)=\sqrt{S_{2}} x_{m} \delta^{M}\left(x-x^{\prime}\right) \\
& \pi_{m}\left(x, x^{\prime}\right)=\frac{1}{\sqrt{S_{2}}} \frac{\partial}{\partial x_{m}} \delta^{M}\left(x-x^{\prime}\right) .
\end{aligned}
$$

The canonical commutation relations 


$$
\begin{gathered}
\left(\begin{array}{cc}
{\left[\phi_{m}, \phi_{m^{\prime}}\right]} & {\left[\phi_{m}, \pi_{m^{\prime}}\right]} \\
{\left[\pi_{m}, \phi_{m^{\prime}}\right]} & {\left[\pi_{m}, \pi_{m^{\prime}}\right]}
\end{array}\right)=\left(\begin{array}{rr}
0 & -1 \\
1 & 0
\end{array}\right) \delta_{m m^{\prime}} \\
\left(m, m^{\prime}=0,1, \ldots, M-1\right)
\end{gathered}
$$

are easily verified. Hence $W_{\boldsymbol{B}}=\bigoplus_{m=0}^{M-1}\left(\boldsymbol{C} \phi_{m} \oplus \boldsymbol{C} \pi_{m}\right)$ is equipped with a symplectic structure. In terms of these free fields, $V_{1}, V_{2}$ are expressed as

$$
\begin{aligned}
& V_{1}=\exp \left(-\sum_{m=0}^{M-1}\left(C_{1} C_{2}^{*} \phi_{m}^{2}-S_{1} S_{2}^{*} \phi_{m} \phi_{m+1}\right)\right) \\
& V_{2}=\sqrt{2 \pi S_{2}^{*}} \exp \left(\frac{\pi}{4} \sum_{m=0}^{M-1}\left(\phi_{m}^{2}+\pi_{m}^{2}\right)\right)
\end{aligned}
$$

To see the second equality we note the following lemma.

Lemma. Put

$$
u_{t}\left(x, x^{\prime} ; c\right)=\frac{c}{\sqrt{2 \pi \sin t}} \exp \left(-\frac{c^{2} \cos t}{2 \sin t}\left(x^{2}+x^{\prime 2}\right)+\frac{c^{2} x x^{\prime}}{\sin t}\right)
$$

for $c>0$. We have then

$$
\left\{\begin{array}{l}
\frac{\partial}{\partial t} u_{t}\left(x, x^{\prime} ; c\right)=\frac{1}{2}\left(c^{2} x^{2}+\frac{1}{c^{2}} \frac{\partial^{2}}{\partial x^{2}}\right) u_{t}\left(x, x^{\prime}\right) \quad(0<t<\pi) \\
u_{+0}\left(x, x^{\prime}\right)=\delta\left(x-x^{\prime}\right)
\end{array}\right.
$$

We omit the proof.

Setting $t=\pi / 2$ in (5.4.19) we obtain the kernel for the operator $\exp \left(\frac{\pi}{4}\left(c^{2} x^{2}\right.\right.$ $\left.+\frac{1}{c^{2}} \frac{\partial^{2}}{\partial x^{2}}\right)$ ), and (5.4.18) follows. We set also $\phi_{m n}=V^{n} \phi_{m} V^{-n}, \pi_{m n}=V^{n} \pi_{m} V^{-n}$ and

$$
\hat{\phi}\left(\theta_{\mu}\right)=\sum_{m=0}^{M-1} e^{-i m \theta_{\mu}} \phi_{m}, \quad \hat{\pi}\left(\theta_{\mu}\right)=\sum_{m=0}^{M-1} e^{-i m \theta_{\mu}} \pi_{m} .
$$

As in Section 5.1, we fix an expectation value $\langle>$ given by

$$
\langle a\rangle=Z_{M N}^{-1} \operatorname{trace}\left(a V^{N}\right), \quad a \in A\left(W_{B}\right) .
$$

Proposition 5.4.2. The table of expectation values for (5.4.21) reads as follows:

$$
\left(\begin{array}{c}
\left\langle\hat{\phi}\left(\theta_{\mu}\right) \hat{\phi}\left(\theta_{\mu^{\prime}}\right)\right\rangle\left\langle\hat{\phi}\left(\theta_{\mu}\right) \hat{\pi}\left(\theta_{\mu^{\prime}}\right)\right\rangle \\
\left\langle\hat{\pi}\left(\theta_{\mu}\right) \hat{\phi}\left(\theta_{\mu^{\prime}}\right)\right\rangle\left\langle\hat{\pi}\left(\theta_{\mu}\right) \hat{\pi}\left(\theta_{\mu^{\prime}}\right)\right\rangle
\end{array}\right)=\frac{1}{2}\left(\begin{array}{cc}
a_{\mu} & -2-b_{\mu} \\
-b_{\mu} & a_{\mu}
\end{array}\right) \cdot M \delta_{\mu,-\mu^{\prime}}
$$

where

$$
a_{\mu}=\frac{1}{N} \sum_{v=0}^{N-1} \frac{S_{2}}{\Delta\left(\theta_{\mu}, \theta_{v}^{\prime}\right)}=a_{-\mu}
$$




$$
b_{\mu}=\frac{1}{N} \sum_{v=0}^{N-1} \frac{S_{2} e^{i \theta_{v}^{\prime}}}{\Delta\left(\theta_{\mu}, \theta_{v}^{\prime}\right)}=b_{-\mu} .
$$

Proof. As an example we evaluate $\left\langle\hat{\pi}\left(\theta_{\mu}\right) \hat{\pi}\left(\theta_{\mu^{\prime}}\right)\right\rangle$. The rest are calculated similarly. By the definition we have

$$
\begin{aligned}
S_{2}\left\langle\pi_{m} \pi_{m^{\prime}}\right\rangle= & Z_{M N}^{-1} \int_{\boldsymbol{R}^{M N}} d x\left(\frac{\partial^{2}}{\partial y_{m} \partial y_{m^{\prime}}} V\left(y, x^{(1)}\right) V\left(x^{(1)}, x^{(2)}\right) \cdots\right. \\
& \left.\cdots V\left(x^{(N-1)}, x^{(0)}\right)\right)\left.\right|_{y=x(0)} \\
= & Z_{M N}^{-1} \int_{R^{M N}} d x e^{-E(x)}\left\{-2 C_{1} C_{2} \delta_{m m^{\prime}}+S_{1}\left(\delta_{m, m^{\prime}-1}+\delta_{m, m^{\prime}+1}\right)\right. \\
& +\left(-2 C_{1} C_{2} x_{m 0}+S_{1}\left(x_{m-1,0}+x_{m+1,0}\right)+S_{2} x_{m 1}\right) \\
& \left.\times\left(-2 C_{1} C_{2} x_{m^{\prime} 0}+S_{1}\left(x_{m^{\prime}-1.0}+x_{m^{\prime}+1,0}\right)+S_{2} x_{m^{\prime} 1}\right)\right\} .
\end{aligned}
$$

Substitution of (5.4.12) shows that the right hand side is equal to

$$
\begin{aligned}
& -2 C_{1} C_{2} \delta_{m, m^{\prime}}+S_{1}\left(\delta_{m, m^{\prime}-1}+\delta_{m, m^{\prime}+1}\right) \\
& +4 \frac{1}{2 M N} \sum_{\mu=0}^{M-1} \sum_{v=0}^{N-1} \frac{e^{i\left(m-m^{\prime}\right) \theta_{\mu}}}{\Delta\left(\theta_{\mu}, \theta_{v}^{\prime}\right)}\left(\Delta\left(\theta_{\mu}, \theta_{1}^{\prime}\right)+\frac{1}{2} S_{2} e^{i \theta_{v}^{\prime}}\right)\left(\Delta\left(\theta_{\mu}, \theta_{v}^{\prime}\right)+\frac{1}{2} S_{2} e^{-i \theta_{v}^{\prime}}\right) \\
& =\frac{S_{2}^{2}}{2} \frac{1}{M N} \sum_{\mu=0}^{M-1} \sum_{v=0}^{N-1} \frac{e^{i\left(m-m^{\prime}\right) \theta_{\mu}}}{\Delta\left(\theta_{\mu}, \theta_{v}^{\prime}\right)} .
\end{aligned}
$$

Taking the Fourier transformation we obtain $\left\langle\hat{\pi}\left(\theta_{\mu}\right) \hat{\pi}\left(\theta_{\mu^{\prime}}\right)\right\rangle$.

Remark. In the limit $N \rightarrow \infty,(5.4 .24)$ tends respectively to $a_{\mu} \rightarrow 1 / \sinh \gamma\left(\theta_{\mu}\right)$ and $b_{\mu} \rightarrow e^{-\gamma\left(\theta_{\mu}\right)} / \sinh \gamma\left(\theta_{\mu}\right)$, so that (5.4.23) simplifies into

$$
\begin{aligned}
\left(\begin{array}{ccc}
\left\langle\hat{\phi}\left(\theta_{\mu}\right) \hat{\phi}\left(\theta_{\mu^{\prime}}\right)\right\rangle & \left\langle\hat{\phi}\left(\theta_{\mu}\right) \hat{\pi}\left(\theta_{\mu^{\prime}}\right)\right\rangle \\
\left\langle\hat{\pi}\left(\theta_{\mu}\right) \hat{\phi}\left(\theta_{\mu^{\prime}}\right)\right\rangle & \left\langle\hat{\pi}\left(\theta_{\mu}\right) \hat{\pi}\left(\theta_{\mu^{\prime}}\right)\right\rangle
\end{array}\right) \\
=\frac{1}{2}\left(\begin{array}{cc}
1 & -e^{\gamma\left(\theta_{\mu}\right)} \\
-e^{-\gamma\left(\theta_{\mu}\right)} & 1
\end{array}\right) \frac{M \delta_{\mu,-\mu^{\prime}}}{\sinh \gamma\left(\theta_{\mu}\right)} .
\end{aligned}
$$

The rotations induced by $V_{1}, V_{2}$ are immediately obtained from (5.4.18).

$$
\begin{array}{ll}
\text { (5.4.25) } & T_{V_{1}} \phi_{m}=\phi_{m}, T_{V_{1}} \pi_{m}=\pi_{m}+2 C_{1} C_{2}^{*} \phi_{m}-S_{1} S_{2}^{*}\left(\phi_{m-1}+\phi_{m+1}\right) \\
& T_{V_{2}} \phi_{m}=\pi_{m}, T_{V_{2}} \pi_{m}=-\phi_{m} \\
& T_{V} \phi_{m}=2 C_{1} C_{2}^{*} \phi_{m}-S_{1} S_{2}^{*}\left(\phi_{m-1}+\phi_{m+1}\right)+\pi_{m} \\
& T_{V} \pi_{m}=-\phi_{m} . \\
(5.4 .26) \quad & T_{V_{1}} \hat{\phi}\left(\theta_{\mu}\right)=\hat{\phi}\left(\theta_{\mu}\right), T_{V_{1}} \hat{\pi}\left(\theta_{\mu}\right)=\hat{\phi}\left(\theta_{\mu}\right) \cdot 2 \cosh \gamma\left(\theta_{\mu}\right)+\hat{\pi}\left(\theta_{\mu}\right) \\
& T_{V_{2}} \hat{\phi}\left(\theta_{\mu}\right)=\hat{\pi}\left(\theta_{\mu}\right), T_{V_{2}} \hat{\pi}\left(\theta_{\mu}\right)=-\hat{\phi}\left(\theta_{\mu}\right) \\
& T_{V} \hat{\phi}\left(\theta_{\mu}\right)=\hat{\phi}\left(\theta_{\mu}\right) \cdot 2 \cosh \gamma\left(\theta_{\mu}\right)+\hat{\pi}\left(\theta_{\mu}\right), T_{V} \hat{\pi}\left(\theta_{\mu}\right)=-\hat{\phi}\left(\theta_{\mu}\right) .
\end{array}
$$

The rotation $T_{V}$ is diagonalized in the following basis: 


$$
\begin{aligned}
& \left\{\begin{array}{l}
\sqrt{2} \phi^{\dagger}\left(-\theta_{\mu}\right)=\hat{\phi}\left(\theta_{\mu}\right)+e^{\gamma\left(\theta_{\mu}\right)} \cdot \hat{\pi}\left(\theta_{\mu}\right) \\
\sqrt{2} \phi\left(\theta_{\mu}\right)=-\hat{\phi}\left(\theta_{\mu}\right)-e^{-\gamma\left(\theta_{\mu}\right)} \cdot \hat{\pi}\left(\theta_{\mu}\right)
\end{array}\right. \\
& \left\{\begin{array}{l}
\sqrt{2} \sinh \gamma\left(\theta_{\mu}\right) \cdot \hat{\phi}\left(\theta_{\mu}\right)=-e^{-\gamma\left(\theta_{\mu}\right)} \phi^{\dagger}\left(-\theta_{\mu}\right)-e^{\gamma\left(\theta_{\mu}\right)} \cdot \phi\left(\theta_{\mu}\right) \\
\sqrt{2} \sinh \gamma\left(\theta_{\mu}\right) \cdot \hat{\pi}\left(\theta_{\mu}\right)=\phi^{\dagger}\left(-\theta_{\mu}\right)+\phi\left(\theta_{\mu}\right) .
\end{array}\right.
\end{aligned}
$$

We have

$$
T_{V} \phi^{\dagger}\left(\theta_{\mu}\right)=e^{-\gamma\left(\theta_{\mu}\right)} \phi^{\dagger}\left(\theta_{\mu}\right), T_{V} \phi\left(\theta_{\mu}\right)=e^{\gamma\left(\theta_{\mu}\right)} \phi\left(\theta_{\mu}\right) .
$$

Moreover from (5.4.23)' and (5.4.27) we have, for $N \rightarrow \infty$,

$$
\begin{aligned}
& \left(\begin{array}{ll}
\left\langle\phi^{\dagger}\left(\theta_{\mu}\right) \phi^{\dagger}\left(\theta_{\mu^{\prime}}\right)\right\rangle & \left\langle\phi^{\dagger}\left(\theta_{\mu}\right) \phi\left(\theta_{\mu^{\prime}}\right)\right\rangle \\
\left\langle\phi\left(\theta_{\mu}\right) \phi^{\dagger}\left(\theta_{\mu^{\prime}}\right)\right\rangle & \left\langle\phi\left(\theta_{\mu}\right) \phi\left(\theta_{\mu^{\prime}}\right)\right\rangle
\end{array}\right)=\left(\begin{array}{ll} 
& 0 \\
1 &
\end{array}\right) \sinh \gamma\left(\theta_{\mu}\right) \cdot M \delta_{\mu,-\mu^{\prime}} \\
& \left(\begin{array}{ll}
{\left[\phi^{\dagger}\left(\theta_{\mu}\right), \phi^{\dagger}\left(\theta_{\mu^{\prime}}\right)\right]} & {\left[\phi^{\dagger}\left(\theta_{\mu}\right), \phi\left(\theta_{\mu^{\prime}}\right)\right]} \\
{\left[\phi\left(\theta_{\mu}\right), \phi^{\dagger}\left(\theta_{\mu^{\prime}}\right)\right]} & {\left[\phi\left(\theta_{\mu}\right), \phi\left(\theta_{\mu^{\prime}}\right)\right]}
\end{array}\right)=\left(\begin{array}{l}
-1 \\
1
\end{array}\right) \sinh \gamma\left(\theta_{\mu}\right) \cdot M \delta_{\mu,-\mu^{\prime}}
\end{aligned}
$$

Hence the expectation value $\langle>$ in this limit coincides with the one induced by the holonomic decomposition $W_{B}=V^{\dagger} \oplus V, V^{\dagger}=\bigoplus_{\mu=0}^{M-1} C \phi^{\dagger}\left(\theta_{\mu}\right), V=\bigoplus_{\mu=0}^{M-1} C \phi\left(\theta_{\mu}\right)$.

Now we return to the correlation function $\rho(\partial \Gamma)$ and define the "spin operator" $s_{B, m n}$ by

$$
\begin{aligned}
s_{B, m}\left(x, x^{\prime}\right) & =\delta\left(x_{0}+x_{0}^{\prime}\right) \cdots \delta\left(x_{m-1}+x_{m-1}^{\prime}\right) \delta\left(x_{m}-x_{m}^{\prime}\right) \cdots \\
s_{B, m n}= & V^{n} s_{B, m} V^{-n} .
\end{aligned}
$$

Then $s_{B, m}$ satisfies the following characteristic commutation relation with the free fields:

$$
\begin{aligned}
s_{B, m} \phi_{m^{\prime}} & = \begin{cases}-\phi_{m^{\prime}} s_{B, m} & \left(0 \leqq m^{\prime} \leqq m-1\right) \\
\phi_{m^{\prime}} s_{B, m} & \left(m \leqq m^{\prime} \leqq M-1\right)\end{cases} \\
s_{B, m} \pi_{m^{\prime}} & = \begin{cases}-\pi_{m^{\prime}} s_{B, m} & \left(0 \leqq m^{\prime} \leqq m-1\right) \\
\pi_{m^{\prime}} s_{B, m} & \left(m \leqq m^{\prime} \leqq M-1\right)\end{cases}
\end{aligned}
$$

Assuming $n_{1} \leqq \cdots \leqq n_{k}$, we have

$$
\begin{aligned}
& \left\langle s_{B, m_{1} n_{1}} \cdots s_{B, m_{k} n_{k}}\right\rangle=Z_{M N}^{-1} \operatorname{trace}\left(V^{n_{1}} s_{B, m_{1}} V^{n_{2}-n_{1}} s_{B, m_{2}} \cdots s_{B, m_{k}} V^{N-n_{k}}\right) \\
& =Z_{M N}^{-1} \int_{R^{M N}} d x V\left(x^{(0)}, x^{(1)}\right) \cdots V\left(x^{\left(n_{1}-1\right)}, x^{\left(n_{1}\right)}\right) V\left(\tilde{x}^{\left(n_{1} ; m_{1}\right)}, x^{\left(n_{1}+1\right)}\right) \\
& \cdots V\left(x^{\left(n_{2}-1\right)}, x^{\left(n_{2}\right)}\right) V\left(\tilde{x}^{\left(n_{2} ; m_{2}\right)}, x^{\left(n_{2}+1\right)}\right) \cdots V\left(x^{(N-1)}, x^{(0)}\right) \\
& =\rho(\partial \Gamma),
\end{aligned}
$$

where $\tilde{x}^{(n ; m)}=\left(-x_{0}^{(n)}, \ldots,-x_{m-1}^{(n)}, x_{m}^{(n)}, \ldots, x_{M-1}^{(n)}\right)$, and $\Gamma$ denotes the polygon shown in Figure 5.4.2: 


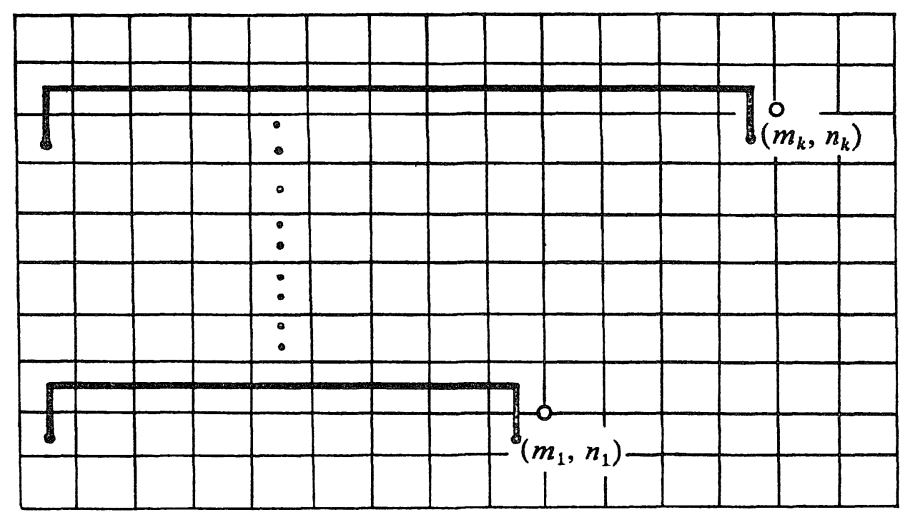

Figure 5.4.2.

Let us compute the norm of $s_{B, m n}$ in the infinite lattice. The free fields are expressed in terms of creation-annihilation operators $\phi^{\dagger}(\theta), \phi(\theta)$ as

$$
\begin{aligned}
\phi_{m n}= & \frac{-1}{\sqrt{2}} \int \underline{d \theta}\left(e^{-i m \theta-(n+1) \gamma(\theta)} \phi^{\dagger}(\theta)\right. \\
& \left.+e^{i m \theta+(n+1) \gamma(\theta)} \phi(\theta)\right), \\
\pi_{m n}= & \frac{-1}{\sqrt{2}} \int \underline{d \theta}\left(e^{-i m \theta-n \gamma(\theta)} \phi^{\dagger}(\theta)\right. \\
& \left.+e^{i m \theta+n \gamma(\theta)} \phi(\theta)\right),
\end{aligned}
$$

where $\underline{d \theta}=d \theta / 2 \pi \sinh \gamma(\theta)$. They satisfy the difference equations

$$
\begin{aligned}
& \phi_{m, n+1}=2 C_{1} C_{2}^{*} \phi_{m n}-S_{1} S_{2}^{*}\left(\phi_{m-1, n}+\phi_{m+1, n}\right)+\pi_{m n} \\
& \pi_{m, n+1}=-\phi_{m n}
\end{aligned}
$$

and in particular

$$
C_{1} C_{2} \phi_{m n}-\frac{1}{2} S_{1}\left(\phi_{m+1, n}+\phi_{m-1, n}\right)-\frac{1}{2} S_{2}\left(\phi_{m, n+1}+\phi_{m, n-1}\right)=0 .
$$

Clearly $\pi_{m n}$ also satisfies (5.4.35).

In the basis $\hat{\phi}(\theta)=\sum_{m \in Z} e^{-i m \theta} \phi_{m}, \hat{\pi}(\theta)=\sum_{m \in Z} e^{-i m \theta} \pi_{m}$ the rotation $T_{s_{00}}=$ $1-2 P$ and the operator $E^{-1}=H^{-1} J$ (in the notation of (A.17), Chapter IV) read

$$
\begin{aligned}
& \left(P \hat{\phi}\left(\theta^{\prime}\right), P \hat{\pi}\left(\theta^{\prime}\right)\right)=\int_{-\pi}^{\pi} \frac{d \theta}{2 \pi}(\hat{\phi}(\theta), \hat{\pi}(\theta)) P\left(\theta, \theta^{\prime}\right) \\
& P\left(\theta, \theta^{\prime}\right)=\frac{e^{-i\left(\theta-\theta^{\prime}\right)}}{1-e^{-i\left(\theta-\theta^{\prime}-i 0\right)}}
\end{aligned}
$$

$$
\begin{aligned}
& \left(E^{-1} \hat{\phi}(\theta), E^{-1} \hat{\pi}(\theta)\right)=(\hat{\phi}(\theta), \hat{\pi}(\theta)) E^{-1}(\theta) \\
& E^{-1}(\theta)=\left(\begin{array}{cc}
-\cosh \gamma(\theta) & 1 \\
-1 & \cosh \gamma(\theta)
\end{array}\right) \frac{1}{\sinh \gamma(\theta)}
\end{aligned}
$$




$$
=-Q^{-1}\left(\begin{array}{cc}
\cosh \gamma(\theta)+1 \\
\cosh \gamma(\theta)-1
\end{array}\right) \frac{1}{\sinh \gamma(\theta)} Q
$$

where $Q=\frac{1}{\sqrt{2}}\left(\begin{array}{rr}1 & 1 \\ 1 & -1\end{array}\right)=Q^{-1}$. In view of the formula

$$
\begin{aligned}
& \frac{\cosh \gamma(\theta)+1}{\sinh \gamma(\theta)}=\frac{\sinh \gamma(\theta)}{\cosh \gamma(\theta)-1}=\frac{1}{\sqrt{\alpha_{1} \alpha_{2}}} c(\theta) c(-\theta) \\
& c(\theta)=\sqrt{\frac{1-\alpha_{1} e^{i \theta}}{1-\alpha_{2}^{-1} e^{i \theta}}}
\end{aligned}
$$

the factorization (A.18) $)_{B}$ is achieved by choosing

$$
\begin{aligned}
& Q X_{+}^{-1} Q^{-1}=-\left(\begin{array}{ll}
\frac{c(\theta)}{\sqrt{\alpha_{1} \alpha_{2}}} & \\
& \frac{\sqrt{\alpha_{1} \alpha_{2}}}{c(\theta)}
\end{array}\right) \\
& Q X_{-} Q^{-1}=\left(\begin{array}{ll}
\frac{1}{c(-\theta)} &
\end{array}\right) .
\end{aligned}
$$

Here we have assumed $T>T_{c}$ for definiteness, but in the case $T<T_{c}$ all the formulas are valid by the replacement $\alpha_{2} \mapsto \alpha_{2}^{-1}$. The kernel $\hat{R}\left(\theta, \theta^{\prime}\right)$ is obtained by applying $(\text { A.19) })_{B}$ :

$$
Q \hat{R}\left(\theta, \theta^{\prime}\right) Q^{-1}=2\left(\begin{array}{ll}
\frac{c(-\theta) c\left(-\theta^{\prime}\right)}{\sqrt{\alpha_{1} \alpha_{2}}} & \\
& -\frac{\sqrt{\alpha_{1} \alpha_{2}}}{c(-\theta) c\left(-\theta^{\prime}\right)}
\end{array}\right) P\left(\theta,-\theta^{\prime}\right) .
$$

The vacuum expectation value $\left\langle s_{B, m n}\right\rangle$ is evaluated by the same method as in Section 5.2, p. 541. Making use of the formula (A.31) and noting $s_{B, m n}^{2}=1$, $\left\langle s_{m n}\right\rangle>0$ in the finite lattice, we obtain

$$
\begin{aligned}
\left\langle s_{B, m n}\right\rangle & =\left(1-S_{1}^{2} S_{2}^{2}\right)^{1 / 8} & \text { if } & T>T_{c}, \\
& =\left(1-S_{1}^{-2} S_{2}^{-2}\right)^{1 / 8} & \text { if } & T<T_{c} .
\end{aligned}
$$

Finally we rewrite the result using the creation-annihilation operators, and obtain the following.

Theorem 5.4.3. The norm of $s_{B, m n}$ has the form

$$
\begin{aligned}
& \operatorname{Nr}\left(s_{B, m n}\right)=\left\langle s_{B, m n}\right\rangle e^{\rho_{m n} / 2} \\
& \rho_{m n}=\iint_{-\pi}^{\pi} \underline{d \theta} \underline{d \theta^{\prime}}\left(\phi^{\dagger}(\theta) \phi(\theta)\right)\left(\begin{array}{ll}
R_{m n}^{--}\left(\theta, \theta^{\prime}\right) & R_{m n}^{-+}\left(\theta, \theta^{\prime}\right) \\
R_{m n}^{+-}\left(\theta, \theta^{\prime}\right) & R_{m n}^{++}\left(\theta, \theta^{\prime}\right)
\end{array}\right)\left(\begin{array}{l}
\phi^{\dagger}\left(\theta^{\prime}\right) \\
\phi\left(\theta^{\prime}\right)
\end{array}\right)
\end{aligned}
$$


where the kernels $R_{m n}^{\sigma \sigma^{\prime}}\left(\theta, \theta^{\prime}\right)$ are given by

$$
\begin{aligned}
R_{m n}^{\sigma \sigma^{\prime}}\left(\theta, \theta^{\prime}\right) & =\frac{1}{2} \frac{1}{\sqrt{\left(1-\alpha_{1} e^{-i \sigma \theta}\right)\left(1-\alpha_{2}^{-1} e^{-i \sigma \theta}\right)\left(1-\alpha_{1} e^{-i \sigma^{\prime} \theta^{\prime}}\right)\left(1-\alpha_{2}^{-1} e^{-i \sigma^{\prime} \theta^{\prime}}\right)}} \\
& \times\left\{\frac{1}{\sqrt{\alpha_{1} \alpha_{2}}}\left(1-\alpha_{1} e^{-i \sigma \theta}\right)\left(1-e^{\sigma \gamma(\theta)}\right)\left(1-\alpha_{1} e^{-\sigma^{\prime} \theta^{\prime}}\right)\left(1-e^{\sigma^{\prime} \gamma\left(\theta^{\prime}\right)}\right)\right. \\
& \left.-\sqrt{\alpha_{1} \alpha_{2}}\left(1-\alpha_{2}^{-1} e^{-i \sigma \theta}\right)\left(1+e^{\sigma \gamma(\theta)}\right)\left(1-\alpha_{2}^{-1} e^{-i \sigma^{\prime} \theta^{\prime}}\right)\left(1+e^{\sigma^{\prime} \gamma\left(\theta^{\prime}\right)}\right)\right\} \\
& \times \frac{1}{1-e^{-i\left(\sigma \theta+\sigma^{\prime} \theta^{\prime}-i 0\right)}} e^{i(m-1)\left(\sigma \theta+\sigma^{\prime} \theta^{\prime}\right)+n\left(\sigma \gamma(\theta)+\sigma^{\prime} \gamma\left(\theta^{\prime}\right)\right)} \\
& \left(\sigma, \sigma^{\prime}= \pm\right)
\end{aligned}
$$

for $T>T_{c}$. If $T<T_{c}$ we replace $\alpha_{2}$ by $\alpha_{2}^{-1}$.

\section{§5.5. The Scaling Limit}

In this section we compute the scaling limit of spin operators of the Ising model as well as of its bosonic counterpart. We shall see that there result the fields $\varphi_{F}, \varphi^{F}$ and $\varphi_{B}$ constructed in the previous chapter [3].

Let us consider a square lattice of unit length $\varepsilon$. By scaling limit we mean the simultaneous limit

$$
T \rightarrow T_{c} \quad \varepsilon \rightarrow 0 ; \quad m, n \rightarrow \infty
$$

where $m \varepsilon$ and $n \varepsilon$ being fixed and finite.

First consider the Ising model above the critical temperature $T>T_{c}$. We set

$$
x^{1}=m \varepsilon, \quad x^{0}=\sqrt{-1} \kappa n \varepsilon .
$$

Here the factor $\sqrt{-1}$ is inserted so that in the limit $\varepsilon \rightarrow 0\left(x^{0}, x^{1}\right) \in \boldsymbol{R}^{2}$ constitutes a coordinate of 2-dimensional Minkowski space-time. The positive constant $\kappa$ will be fixed later. Choose constants $\alpha, \mu, \mu^{\prime}$ so that $0<\alpha<1, \mu>0$. Let the interaction strengths be given through

$$
\alpha_{1}=\alpha+\varepsilon \mu^{\prime}, \quad \alpha_{2}=1+\varepsilon \mu
$$

where $\alpha_{1}, \alpha_{2}$ are defined in (5.1.29)-(5.1.30). We also set

$$
p^{1}=\theta / \varepsilon \text {. }
$$

In the limit $\varepsilon \rightarrow 0$ we have then the following. 


$$
\begin{gathered}
S_{1}=\frac{2 \sqrt{\alpha}}{1-\alpha}+\varepsilon \frac{1+\alpha}{\sqrt{\alpha}(1-\alpha)^{2}}\left(\mu^{\prime}-\alpha \mu\right)+O\left(\varepsilon^{2}\right), \\
C_{1}=\frac{1+\alpha}{1-\alpha}+\varepsilon \frac{2}{(1-\alpha)^{2}}\left(\mu^{\prime}-\alpha \mu\right)+O\left(\varepsilon^{2}\right), \\
S_{2}^{*}=\frac{2 \sqrt{\alpha}}{1-\alpha}+\varepsilon \frac{1+\alpha}{\sqrt{\alpha}(1-\alpha)^{2}}\left(\mu^{\prime}+\alpha \mu\right)+O\left(\varepsilon^{2}\right), \\
C_{2}^{*}=\frac{1+\alpha}{1-\alpha}+\varepsilon \frac{2}{(1-\alpha)^{2}}\left(\mu^{\prime}+\alpha \mu\right)+O\left(\varepsilon^{2}\right) . \\
a(\theta)^{ \pm 1} \sinh \gamma(\theta)=\varepsilon \frac{2 \sqrt{\alpha}}{1-\alpha}\left(\mu \mp i p^{1}\right)+O\left(\varepsilon^{2}\right), \\
\quad \sinh \gamma(\theta)=\varepsilon \frac{2 \sqrt{\alpha}}{1-\alpha} \sqrt{\mu^{2}+\left(p^{1}\right)^{2}}+O\left(\varepsilon^{2}\right) .
\end{gathered}
$$

We set

$$
\kappa=\frac{2 \sqrt{\alpha}}{1-\alpha} .
$$

We introduce a parameter $u$ and an operator $\psi(u)$ by

$$
\begin{array}{ll}
u^{ \pm 1}=\frac{\sqrt{\mu^{2}+\left(p^{1}\right)^{2}} \pm p^{1}}{\mu}, & \psi(u)=\frac{\psi(\theta)}{\sqrt{\kappa}} \quad \text { for } u>0, \\
u^{ \pm 1}=\frac{-\sqrt{\mu^{2}+\left(p^{1}\right)^{2}} \pm p^{1}}{\mu}, & \psi(u)=\frac{\psi^{\dagger}(-\theta)}{\sqrt{\kappa}} \quad \text { for } u<0 .
\end{array}
$$

Then we have in the limit $\varepsilon \rightarrow 0$

$$
\begin{gathered}
\left\langle\psi(u), \psi\left(u^{\prime}\right)\right\rangle=2 \pi|u| \delta\left(u+u^{\prime}\right), \\
e^{-n P^{0}}=\exp \left(i x^{0} \int_{0}^{\infty} \underline{d u} p^{0} \psi(-u) \psi(u)\right), \\
e^{-i m P^{1}}=\exp \left(-i x^{1} \int_{0}^{\infty} \underline{d u} p^{1} \psi(-u) \psi(u)\right),
\end{gathered}
$$

where $\underline{d u}=d u / 2 \pi|u|$ and $p^{0}= \pm \sqrt{\mu^{2}+\left(p^{1}\right)^{2}}$ if $u \gtrless 0$.

From Theorem 5.2.3 we have the following.

Theorem 5.5.1. In the limit $\varepsilon \rightarrow 0$, we have

$$
\mathrm{Nr}\left(s_{m n}\right)=\left(2 \frac{1+\alpha}{1-\alpha} \mu \varepsilon\right)^{1 / 8} \mathrm{Nr}\left(\varphi^{F}(x)\right)+O\left(\varepsilon^{9 / 8}\right),
$$

where $\varphi^{F}(x)$ is given in (4.6.2) of [3]. Namely

$$
\begin{aligned}
& \operatorname{Nr}\left(\varphi^{F}(x)\right)=\psi_{0}(x) e^{\rho_{F}(x) / 2}, \\
& \psi_{0}(x)=\int_{-\infty}^{\infty} \underline{d u} e^{-i \mu\left(x^{-} u+x^{+} u^{-1}\right)} \psi(u),
\end{aligned}
$$




$$
\rho_{F}(x)=\iint_{-\infty}^{\infty} \underline{d u} \underline{d u^{\prime}} \frac{-i\left(u-u^{\prime}\right)}{u+u^{\prime}-i 0} e^{-i \mu\left(x^{-}\left(u+u^{\prime}\right)+x^{+}\left(u^{-1}+u^{\prime}-1\right)\right)} \psi(u) \psi\left(u^{\prime}\right) .
$$

Corollary 5.5.2. In the limit $\varepsilon \rightarrow 0$, we have

$(5.5 .14)_{T>T_{c}}$

$$
\begin{aligned}
& \rho_{k}\left(\left(m_{1}, n_{1}\right), \ldots,\left(m_{k}, n_{k}\right)\right) \\
= & \left(2 \frac{1+\alpha}{1-\alpha} \mu \varepsilon\right)^{k / 8}\left\langle\varphi^{F}\left(x^{(1)}\right) \cdots \varphi^{F}\left(x^{(k)}\right)\right\rangle+O\left(\varepsilon^{k / 8+1}\right),
\end{aligned}
$$

where $x^{(j)}=\left(m_{j} \varepsilon, \sqrt{-1} \kappa n_{j} \varepsilon\right)(j=1, \ldots, k)$ and $\left\langle\varphi^{F}\left(x^{(1)}\right) \cdots \varphi^{F}\left(x^{(k)}\right)\right\rangle$ is given by (4.6.57), (4.6.59), (4.6.70) and (4.6.71).

Not only the spin operator but also the auxiliary operators $p_{m n}$ and $q_{m n}$ have the scaling limits.

Theorem 5.5.3. In the limit $\varepsilon \rightarrow 0$, we have

$$
\begin{aligned}
& p_{m n}=\sqrt{\mu \varepsilon / 2}\left(\psi_{+}(x)+\psi_{-}(x)\right), \\
& q_{m n}=-i \sqrt{\mu \varepsilon / 2}\left(\psi_{+}(x)-\psi_{-}(x)\right),
\end{aligned}
$$

where

$$
\psi_{ \pm}(x)=\int_{-\infty}^{\infty} d \underline{d} \sqrt{0+i u}^{ \pm 1} e^{i \mu\left(x^{-} u+x^{+} u^{-1}\right)} \psi(u)
$$

Remark. The difference equation (5.2.27) goes to the 2-dimensional Dirac equation in the limit. In fact setting

$$
\begin{aligned}
& v_{m n}^{( \pm)}=v^{( \pm)}(x), \\
& \frac{v_{m, n+1}^{( \pm)}-v_{m n}^{( \pm)}}{\varepsilon \kappa}=i \frac{\partial v^{( \pm)}}{\partial x^{0}}(x), \\
& \frac{v_{m+1, n}^{( \pm)}-v_{m n}^{( \pm)}}{\varepsilon}=\frac{\partial v^{( \pm)}}{\partial x^{1}}(x),
\end{aligned}
$$

we have

$(5.5 .18)_{T>T_{c}}$

$$
\begin{aligned}
& i \frac{\partial v^{(+)}}{\partial x^{0}}=\frac{\partial v^{(-)}}{\partial x^{1}}+\mu v^{(-)} \\
& i \frac{\partial v^{(-)}}{\partial x^{0}}=-\frac{\partial v^{(+)}}{\partial x^{1}}+\mu v^{(+)}
\end{aligned}
$$

Taking (5.5.15) into account we set

$$
w_{ \pm}=v^{(+)} \pm i v^{(-)} .
$$

$(5.5 .18)_{T>T_{c}}$ is transformed into (4.2.42) for $w_{ \pm}$

The case $T<T_{c}$ is similarly treated. We set 


$$
\alpha_{1}=\alpha+\varepsilon \mu^{\prime}, \quad \alpha_{2}=1-\varepsilon \mu .
$$

In (5.5.5) and (5.5.6) $\mu$ should be replaced by $-\mu$, but (5.5.8) is unchanged.

Theorem 5.5.4. In the limit $\varepsilon \rightarrow 0$, we have

$$
\operatorname{Nr}\left(\vec{s}_{m n}\right)=\left(2 \frac{1+\alpha}{1-\alpha} \mu \varepsilon\right)^{1 / 8} \operatorname{Nr}\left(\varphi_{F}(x)\right)+O\left(\varepsilon^{9 / 8}\right),
$$

where $\varphi_{F}(x)$ is given in (4.2.45). Namely

$$
\begin{gathered}
\operatorname{Nr}\left(\varphi_{F}(x)\right)=e^{\rho_{F}(x) / 2}, \\
\rho_{F}(x)=\iint_{-\infty}^{\infty} \underline{d u} \underline{d u^{\prime}} \frac{-i\left(u-u^{\prime}\right)}{u+u^{\prime}-i 0} e^{-i \mu\left(x^{-}\left(u+u^{\prime}\right)+x^{+}\left(u^{-1}+u^{\prime-1}\right)\right)} \psi(u) \psi\left(u^{\prime}\right) .
\end{gathered}
$$

Corollary 5.5.5. In the limit $\varepsilon \rightarrow 0$, we have

$$
\begin{aligned}
& \rho_{k}\left(\left(m_{1}, n_{1}\right), \ldots,\left(m_{k}, n_{k}\right)\right) \\
= & \left(2 \frac{1+\alpha}{1-\alpha} \mu \varepsilon\right)^{k / 8}\left\langle\varphi_{F}\left(x^{(1)}\right) \cdots \varphi_{F}\left(x^{(k)}\right)\right\rangle+O\left(\varepsilon^{k / 8+1}\right)
\end{aligned}
$$

where $x^{(j)}=\left(m_{j} \varepsilon, \sqrt{-1} \kappa n_{j} \varepsilon\right)(j=1, \ldots, k)$ and $\left\langle\varphi_{F}\left(x^{(1)}\right) \cdots \varphi_{F}\left(x^{(k)}\right)\right\rangle$ is given by (4.6.70).

Theorem 5.5.6. In the limit $\varepsilon \rightarrow 0$, we have

$$
\begin{aligned}
& p_{m n}=\sqrt{\mu \varepsilon / 2}\left(\psi_{+}(x)-\psi_{-}(x)\right), \\
& q_{m n}=-i \sqrt{\mu \varepsilon / 2}\left(\psi_{+}(x)+\psi_{-}(x)\right) .
\end{aligned}
$$

Remark. The limit of the difference equation reads

$$
\begin{aligned}
& i \frac{\partial v^{(+)}}{\partial x^{0}}=\frac{\partial v^{(-)}}{\partial x^{1}}-\mu v^{(-)} \\
& i \frac{\partial v^{(-)}}{\partial x^{0}}=-\frac{\partial v^{(+)}}{\partial x^{1}}-\mu v^{(+)}
\end{aligned}
$$

If we set

$$
w_{ \pm}= \pm v^{(+)}+i v^{(-)}
$$

$(5.5 .18)_{T<T_{c}}$ is transformed into (4.2.42).

Now we turn to the bosonic model. Using the parametrization (5.5.3) for $T>T_{c}$ or $(5.5 .3)^{\prime}$ for $T<T_{c}$, we see from (5.4.43) that in this case

$$
\begin{aligned}
R_{B, m n}^{\sigma \sigma^{\prime}}( & \left.\theta, \theta^{\prime}\right)=\frac{2 \sqrt{\alpha}}{1-\alpha} \cdot \frac{-i}{\sigma p^{1}+\sigma^{\prime} p^{1 \prime}-i 0} \\
& \times\left\{\sqrt{\mu-i \sigma p^{1}} \sqrt{\mu-i \sigma^{\prime} p^{1 \prime}}-\sqrt{\mu+i \sigma p^{1}} \sqrt{\mu+i \sigma^{\prime} p^{1 \prime}}\right\}+O(\varepsilon) .
\end{aligned}
$$


Rewriting this in terms of the parameter $u$ in (5.5.8) and

$$
\phi(u)= \begin{cases}\frac{\phi(\theta)}{\sqrt{\kappa}} & (u>0) \\ \frac{\phi^{\dagger}(-\theta)}{\sqrt{\kappa}} & (u<0),\end{cases}
$$

we thus obtain

Theorem 5.5.7. In the limit $\varepsilon \rightarrow 0$,

$$
\operatorname{Nr}\left(s_{B, m n}\right)=\left(2 \frac{1+\alpha}{1-\alpha} \mu \varepsilon\right)^{1 / 8} \mathrm{Nr}\left(\varphi_{B}(x)\right)+O\left(\varepsilon^{9 / 8}\right)
$$

where $\varphi_{B}(x)$ is given by ((4.1.66) in IV [3])

$$
\begin{aligned}
& \operatorname{Nr}\left(\varphi_{B}(x)\right)=e^{\rho_{B}(x) / 2} \\
& \rho_{B}(x)=\iint_{-\infty}^{+\infty} \underline{d u} \underline{d u^{\prime}} \frac{-2 \sqrt{u-i 0} \sqrt{u^{\prime}-i 0}}{u+u^{\prime}-i 0} \\
& \times e^{-i \mu\left(x^{-}\left(u+u^{\prime}\right)+x^{+}\left(u^{-1}+u^{\prime}-1\right)\right)} \phi(u) \phi\left(u^{\prime}\right) .
\end{aligned}
$$

Hence the $k$-point functions of $s_{B, m n}$ are scaled to give those of $\varphi_{B}(x)$.

Theorem 5.5.8. We have

$$
\begin{aligned}
& \phi_{m n}=-\frac{1}{\sqrt{2 \kappa}} \phi(x)+O(\varepsilon) \\
& \pi_{m n}+\phi_{m n}=-\frac{\sqrt{\kappa}}{2 \sqrt{2}} \varepsilon i \frac{\partial \phi}{\partial x^{0}}(x)+O\left(\varepsilon^{2}\right)
\end{aligned}
$$

where

$$
\phi(x)=\int_{-\infty}^{+\infty} d u e^{-i \mu\left(x^{-} u+x^{+} u^{-1}\right)} \phi(u) .
$$

The proof is straightforward from (5.4.33).

\section{$\S 5.6$. The One-Dimensional $X Y$ Model}

The one-dimensional $X Y$ model is described by the Hamiltonian

$$
\mathscr{H}^{\prime}{ }_{M}=-\frac{1}{4} \sum_{m=0}^{M-1}\left\{(1+\gamma) \sigma_{m}^{x} \sigma_{m+1}^{x}+(1-\gamma) \sigma_{m}^{y} \sigma_{m+1}^{y}+2 h \sigma_{m}^{z}\right\}
$$

where $\sigma_{m}^{*}=I_{2} \otimes \cdots \otimes \stackrel{m}{\sigma}^{*} \otimes \cdots \otimes I_{2} \quad(*=x, y, z)$. Here $\sigma^{x}, \sigma^{y}, \sigma^{z}$ are the Pauli matrices

$$
\left(\begin{array}{ll}
1 & 1 \\
1 &
\end{array}\right),\left(\begin{array}{ll}
i^{-i} \\
i^{2}
\end{array}\right),\left(\begin{array}{ll}
1 & \\
& -1
\end{array}\right)
$$


respectively. We set

$$
\begin{aligned}
& p_{m}=\sigma_{0}^{z} \cdots \sigma_{m-1}^{z} \sigma_{m}^{x} \\
& q_{m}=i \sigma_{0}^{z} \cdots \sigma_{m-1}^{z} \sigma_{m}^{y}
\end{aligned}
$$

and define $\hat{p}\left(\theta_{\mu}\right)$ and $\hat{q}\left(\theta_{\mu}\right)$ by (5.1.22). We adopt the modified Hamiltonian.

$$
\begin{aligned}
\mathscr{H}_{M}= & \frac{1}{4} \sum_{m=0}^{M-1}\left\{(1+\gamma) q_{m} p_{m+1}-(1-\gamma) p_{m} q_{m+1}-2 h q_{m} p_{m}\right\} \\
= & \frac{1}{4 M} \sum_{\mu=0}^{M-1}\left\{(1+\gamma) \hat{q}\left(\theta_{\mu}\right) \hat{p}\left(-\theta_{\mu}\right) e^{-i \theta_{\mu}}-(1-\gamma) \hat{p}\left(\theta_{\mu}\right) \hat{q}\left(-\theta_{\mu}\right) e^{-i \theta_{\mu}}\right. \\
& \left.-2 h \hat{q}\left(\theta_{\mu}\right) \hat{p}\left(-\theta_{\mu}\right)\right\} .
\end{aligned}
$$

$\mathscr{H}_{M}$ induces an infinitesimal orthogonal transformation on $W_{M}=\sum_{\mu=0}^{M-1} \boldsymbol{C} \hat{p}\left(\theta_{\mu}\right)+$ $\sum_{\mu=0}^{M-1} C \hat{q}\left(\theta_{\mu}\right)$

$$
\begin{aligned}
& \left(\left[\mathscr{H}_{M}, \hat{p}\left(\theta_{\mu}\right)\right],\left[\mathscr{H}_{M}, \hat{q}\left(\theta_{\mu}\right)\right]\right) \\
& \quad=\left(\hat{p}\left(\theta_{\mu}\right), \hat{q}\left(\theta_{\mu}\right)\right)\left(\begin{array}{ll}
A_{+}\left(\theta_{\mu}\right) \\
A_{-}\left(\theta_{\mu}\right)
\end{array}\right) \\
& A_{ \pm}(\theta)=\cos \theta-h \pm i \gamma \sin \theta .
\end{aligned}
$$

We set

$$
\begin{aligned}
& E(\theta)=\sqrt{A_{+}(\theta) A_{-}(\theta)}=\sqrt{(\cos \theta-h)^{2}+\gamma^{2} \sin ^{2} \theta}, \\
& \alpha_{ \pm}=\frac{h \pm \sqrt{h^{2}+\gamma^{2}-1}}{1-\gamma} .
\end{aligned}
$$

Then we have

$$
A_{ \pm}(\theta)=\frac{1-\gamma}{2} e^{ \pm i \theta}\left(e^{\mp i \theta}-\alpha_{+}\right)\left(e^{\mp i \theta}-\alpha_{-}\right) .
$$

We distinguish the following three phases. (Figure 5.6.1.)

$$
\begin{array}{ll}
\mathscr{R}_{1}: & \gamma>0, h>1 \text { where }\left|\alpha_{+}^{-1}\right|,\left|\alpha_{-}\right|<1, \\
\mathscr{R}_{2}: & \gamma>0,-1<h<1 \text { where }\left|\alpha_{+}^{-1}\right|,\left|\alpha_{-}^{-1}\right|<1, \\
\mathscr{R}_{3}: & \gamma>0, h<-1 \quad \text { where }\left|\alpha_{+}\right|,\left|\alpha_{-}^{-1}\right|<1 .
\end{array}
$$

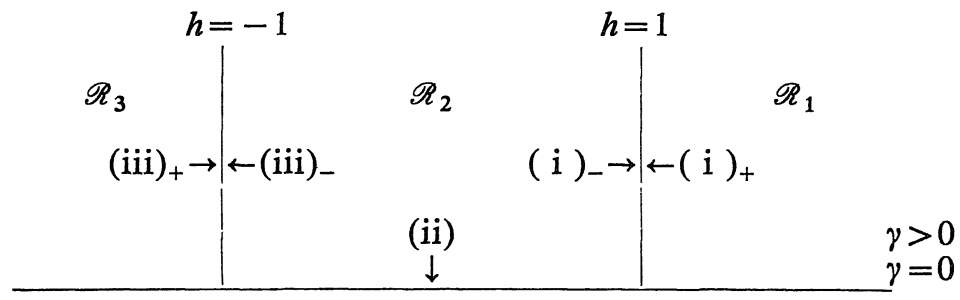

Figure 5.6.1. 
We shall diagonalize $\mathscr{H}_{M}$ in each phase. The results are as follows.

$$
\begin{aligned}
& \mathscr{H}_{M, \text { ren }}=\frac{1}{M} \sum_{\mu=0}^{M-1} E\left(\theta_{\mu}\right) \hat{\psi}_{1}^{\dagger}\left(\theta_{\mu}\right) \hat{\psi}_{1}\left(\theta_{\mu}\right), \\
& 2 \hat{\psi}_{1}^{\dagger}\left(-\theta_{\mu}\right)=\sqrt{a_{1}\left(\theta_{\mu}\right)} \hat{p}\left(\theta_{\mu}\right)-\sqrt{a_{1}\left(\theta_{\mu}\right)^{-1}} \hat{q}\left(\theta_{\mu}\right), \\
& 2 \hat{\psi}_{1}\left(\theta_{\mu}\right)=\sqrt{a_{1}\left(\theta_{\mu}\right)} \hat{p}\left(\theta_{\mu}\right)+\sqrt{a_{1}\left(\theta_{\mu}\right)^{-1}} \hat{q}\left(\theta_{\mu}\right), \\
& a_{1}(\theta)=b_{1}(\theta) / b_{1}(-\theta), \quad b_{1}(\theta)=\sqrt{\frac{1-\alpha_{-} e^{i \theta}}{1-\alpha_{+}^{-1} e^{i \theta}}}, \\
& -A_{ \pm}(\theta)=E(\theta) a_{1}(\theta)^{ \pm 1} \text {. } \\
& (5.6 .9)_{\mathscr{R}_{2}} \quad \mathscr{H}_{M, \text { ren }}=\frac{1}{M} \sum_{\mu=0}^{M-1} E\left(\theta_{\mu}\right) \hat{\psi}_{2}^{\dagger}\left(\theta_{\mu}\right) \hat{\psi}_{2}\left(\theta_{\mu}\right) \text {, } \\
& 2 \hat{\psi}_{2}^{\dagger}\left(-\theta_{\mu}\right)=\sqrt{a_{2}\left(\theta_{\mu}\right)} e^{i \theta_{\mu}} \hat{p}\left(\theta_{\mu}\right)+\sqrt{a_{2}\left(\theta_{\mu}\right)^{-1}} \hat{q}\left(\theta_{\mu}\right), \\
& 2 \hat{\psi}_{2}\left(\theta_{\mu}\right)=\sqrt{a_{2}\left(\theta_{\mu}\right)} e^{i \theta_{\mu}} \hat{p}\left(\theta_{\mu}\right)-\sqrt{a_{2}\left(\theta_{\mu}\right)^{-1}} \hat{q}\left(\theta_{\mu}\right) \text {, } \\
& a_{2}(\theta)=b_{2}(\theta) / b_{2}(-\theta), \quad b_{2}(\theta)=\frac{1}{\sqrt{\left(1-\alpha_{+}^{-1} e^{i \theta}\right)\left(1-\alpha_{-}^{-1} e^{i \theta}\right)}}, \\
& A_{ \pm}(\theta)=E(\theta)\left(a_{2}(\theta) e^{i \theta}\right)^{ \pm 1} \text { 。 } \\
& 2 \hat{\psi}_{3}^{\dagger}\left(-\theta_{\mu}\right)=\sqrt{a_{3}\left(\theta_{\mu}\right)} \hat{p}\left(\theta_{\mu}\right)+\sqrt{a_{3}\left(\theta_{\mu}\right)^{-1}} \hat{q}\left(\theta_{\mu}\right), \\
& 2 \hat{\psi}_{3}\left(\theta_{\mu}\right)=\sqrt{a_{3}\left(\theta_{\mu}\right)} \hat{p}\left(\theta_{\mu}\right)-\sqrt{a_{3}\left(\theta_{\mu}\right)^{-1}} \hat{q}\left(\theta_{\mu}\right) \text {, } \\
& a_{3}(\theta)=b_{3}(\theta) / b_{3}(-\theta), \quad b_{3}(\theta)=\sqrt{\frac{1-\alpha_{+} e^{i \theta}}{1-\alpha_{-}^{-1} e^{i \theta}}}, \\
& A_{ \pm}(\theta)=E(\theta) a_{3}(\theta)^{ \pm 1} \text {. }
\end{aligned}
$$$$
(5.6 .9)_{\mathscr{R}_{3}} \quad \mathscr{H}_{M, \text { ren }}=\frac{1}{M} \sum_{\mu=0}^{M-1} E\left(\theta_{\mu}\right) \hat{\psi}_{3}^{\dagger}\left(\theta_{\mu}\right) \hat{\psi}_{3}\left(\theta_{\mu}\right) \text {, }
$$

Here, $\mathscr{H}_{M, \text { ren }}$ means the renormalized Hamiltonian obtained by subtracting the zero point energy from $\mathscr{H}_{M}$.

Now we go to the limit $M \rightarrow \infty$, and compute the ground state averages for products of spin operators. In other words we compute correlation functions for $\sigma_{m}^{x}, \sigma_{m}^{y}$ and $\sigma_{m}^{z}$ with respect to the vacuum expectation given by (5.2.5) with $\hat{\psi}^{\dagger}(\theta)=\hat{\psi}_{j}^{\dagger}(\theta), \hat{\psi}(\theta)=\hat{\psi}_{j}(\theta)(j=1,2,3)$ in each phase $\mathscr{R}_{1} \sim \mathscr{R}_{3}$. Since we have product formulas $(\S 1.4[1])$, it is sufficient to compute the norms of $\sigma_{m}^{x}$ and $\sigma_{m}^{y}$. ( $\sigma_{m}^{z}$ is trivial, since $\sigma_{m}^{z}=q_{m} p_{m}$ ) In general we shall compute

$$
\sigma_{m n}^{*}=e^{i n P^{0}-i m P^{1}} \sigma_{0}^{*} e^{-i n P^{0}+i m P^{1}}
$$

for $m \in \mathbb{Z}$ and $n \in \mathbb{R}$. Here we have set

$$
\begin{aligned}
P^{0} & =\int_{-\pi}^{\pi} \frac{d \theta}{2 \pi} E(\theta) \hat{\psi}^{\dagger}(\theta) \hat{\psi}(\theta), \\
P^{1} & =\int_{-\pi}^{\pi} \frac{d \theta}{2 \pi} \theta \hat{\psi}^{\dagger}(\theta) \hat{\psi}(\theta) .
\end{aligned}
$$


The results are as follows.

$$
\begin{aligned}
& \operatorname{Nr}\left(\sigma_{m n}^{x}\right)=\operatorname{Nr}\left(p_{m n} t_{m n}\right)=\psi_{1, m n}^{x} \operatorname{Nr}\left(t_{m n}\right), \\
& \psi_{1, m n}^{x}=\int_{-\pi}^{\pi} \frac{d \theta}{2 \pi} \frac{1}{\left|b_{1}(\theta)\right|}\left(e^{-i m \theta+i n E(\theta)} \hat{\psi}_{1}^{\dagger}(\theta)+e^{i m \theta-i n E(\theta)} \hat{\psi}_{1}(\theta)\right), \\
& \operatorname{Nr}\left(i \sigma_{m n}^{y}\right)=\operatorname{Nr}\left(q_{m n} t_{m n}\right)=\psi_{1, m n}^{y} \operatorname{Nr}\left(t_{m n}\right), \\
& \psi_{1, m n}^{y}=\int_{-\pi}^{\pi} \frac{d \theta}{2 \pi}\left|b_{1}(\theta)\right|\left(-e^{-i m \theta+i n E(\theta)} \hat{\psi}_{1}^{\dagger}(\theta)+e^{i m \theta-i n E(\theta)} \hat{\psi}_{1}(\theta)\right), \\
& \operatorname{Nr}\left(t_{m n}\right)=\left\langle t_{m n}\right\rangle e^{\rho_{1, m n} / 2}, \\
& \left\langle t_{m n}\right\rangle=\left(\frac{h^{2}-1}{h^{2}+\gamma^{2}-1}\right)^{1 / 8} .
\end{aligned}
$$

$\rho_{j, m n}(j=1,2,3)$ is given by (5.2.17) and (5.2.18) with $\hat{\psi}^{\dagger}(\theta), \hat{\psi}(\theta), b(\theta)$ and $\gamma(\theta)$ replaced by $\hat{\psi}_{j}^{\dagger}(\theta), \hat{\psi}_{j}(\theta), b_{j}(\theta)$ and $-i E(\theta)$, respectively. $\psi_{j, m n}^{x}$ and $\psi_{j, m n}^{y}$ are similarly defined as $\psi_{1, m n}^{x}$ and $\psi_{1, m n}^{y}$ with $b_{1}(\theta), \hat{\psi}_{1}^{\dagger}(\theta)$ and $\hat{\psi}_{1}(\theta)$ replaced by $b_{j}(\theta), \hat{\psi}_{j}^{\dagger}(\theta)$ and $\hat{\psi}_{j}(\theta)$, respectively. We note that $t_{m 0}$ induces the rotation

$$
\begin{aligned}
T_{t_{m 0}} p_{m^{\prime}} & = \begin{cases}p_{m^{\prime}} & m^{\prime} \geqq m \\
-p_{m^{\prime}} & m^{\prime} \leqq m-1,\end{cases} \\
T_{t_{m 0}} q_{m^{\prime}} & = \begin{cases}q_{m^{\prime}} & \mathrm{m}^{\prime} \geqq m \\
-q_{m^{\prime}} & m^{\prime} \leqq m-1,\end{cases}
\end{aligned}
$$

and satisfies $t_{m_{1} 0} t_{m_{2} 0}=q_{m_{1}} p_{m_{1}} \cdots q_{m_{2}-1} p_{m_{2}-1}\left(m_{1}<m_{2}\right)$.

As in the case $T<T_{c}$ of the Ising lattice, in the phase $\mathscr{R}_{2}$ we consider $\bar{\sigma}_{m}^{x}=$ $\sigma_{0}^{x} \sigma_{m}^{x}$ and $\bar{\sigma}_{m}^{y}=\sigma_{0}^{x} \sigma_{m}^{y}$ for the finite lattice, and then take the limit.

$$
\begin{aligned}
& \operatorname{Nr}\left(\bar{\sigma}_{m n}^{x}\right)=\left\langle\bar{\sigma}_{m n}^{x}\right\rangle e^{\rho_{2, m n} / 2}, \\
& \left\langle\bar{\sigma}_{m n}^{x}\right\rangle=\sqrt{2}\left(\frac{\gamma^{2}\left(1-h^{2}\right)}{(1+\gamma)^{4}}\right)^{1 / 8}, \\
& \operatorname{Nr}\left(i \bar{\sigma}_{m n}^{y}\right)=\operatorname{Nr}\left(q_{m n} p_{m n} \bar{\sigma}_{m n}^{x}\right)=\psi_{2, m n}^{y} \psi_{2, m-1, n}^{y} \operatorname{Nr}\left(\bar{\sigma}_{m n}^{x}\right) . \\
& \operatorname{Nr}\left(\sigma_{m n}^{x}\right)=\operatorname{Nr}\left(p_{m n} t_{m n}^{\prime}\right)=\psi_{3, m n}^{x} \operatorname{Nr}\left(t_{n m}^{\prime}\right), \\
& \operatorname{Nr}\left(i \sigma_{m n}^{y}\right)=\operatorname{Nr}\left(q_{m n} t_{m n}^{\prime}\right)=-\psi_{3, m n}^{y} \operatorname{Nr}\left(t_{m n}^{\prime}\right), \\
& \operatorname{Nr}\left(t_{m n}^{\prime}\right)=\left\langle t_{m n}^{\prime}\right\rangle e^{\rho_{3, m n} / 2}, \\
& \left\langle t_{m n}^{\prime}\right\rangle=(-)^{m}\left(\frac{h^{2}-1}{h^{2}+\gamma^{2}-1}\right)^{1 / 8} .
\end{aligned}
$$

The factor $(-)^{m}$ comes from the fact that not the limit $\lim _{m \rightarrow \infty}\left\langle q_{0} p_{0} \cdots\right.$ $\left.q_{m-1} p_{m-1}\right\rangle$ but the limit $\lim _{m \rightarrow \infty}\left\langle q_{0} p_{0} \cdots q_{m-1} p_{m-1}\right\rangle(-)^{m}=\left(\frac{h^{2}-1}{h^{2}+\gamma^{2}-1}\right)^{\frac{1}{4}}$ exists. We note that $t_{m 0}^{\prime}$ induces the same rotation as (5.6.13) and satisfies $t_{m_{1} 0}^{\prime} t_{m_{2} 0}^{\prime}=$ $q_{m_{1}} p_{m_{1}} \cdots q_{m_{2}-1} p_{m_{2}-1}\left(m_{1}<m_{2}\right)$. 
Infinite series expressions for correlation functions are obtained by direct application of the product formula ( $\$ 1.4$ [1] and Appendix of IV [3]). For our purpose it suffices to consider operators of the following form:

$$
\begin{gathered}
\operatorname{Nr}\left(g_{m n}\right)=\left\langle g_{m n}\right\rangle e^{\rho_{m n} / 2}, \\
\rho_{m n}=\sum_{\sigma, \sigma^{\prime}= \pm} \iint \frac{d \theta}{2 \pi} \frac{d \theta^{\prime}}{2 \pi} R^{\sigma, \sigma^{\prime}}\left(\theta, \theta^{\prime}\right) e^{i m\left(\sigma \theta+\sigma^{\prime} \theta^{\prime}\right)-i n\left(\sigma E(\theta)+\sigma^{\prime} E\left(\theta^{\prime}\right)\right)} \psi_{\sigma}(\theta) \psi_{\sigma^{\prime}}\left(\theta^{\prime}\right),
\end{gathered}
$$

$$
\begin{aligned}
& \operatorname{Nr}\left(g_{m n}^{(j) \prime}\right)=\left\langle g_{m n}\right\rangle w_{m n}^{(j)} e^{\rho_{m n} / 2}, \\
& \operatorname{Nr}\left(g_{m n}^{\prime \prime}\right)=\left\langle g_{m n}\right\rangle w_{m n}^{(1)} w_{m n}^{(2)} e^{\rho_{m n} / 2}, \\
& w_{m n}^{(j)}=\sum_{\sigma= \pm} \int \frac{d \theta}{2 \pi} c^{(j)}(\theta) e^{i m \sigma \theta-i n \sigma E(\theta)} \psi_{\sigma}(\theta) \quad(j=1,2)
\end{aligned}
$$

where $\psi_{-}(\theta)=\psi^{\dagger}(\theta), \psi_{+}(\theta)=\psi(\theta)$ satisfy $\left[\psi(\theta), \psi^{\dagger}\left(\theta^{\prime}\right)\right]_{+}=2 \pi \delta\left(\theta-\theta^{\prime}\right)$. We have then

$$
\begin{aligned}
& \left\langle g_{m_{1} n_{1}} \cdots g_{m_{k} n_{k}}\right\rangle=\left\langle g_{m_{1} n_{1}}\right\rangle \cdots\left\langle g_{m_{k} n_{k}}\right\rangle \exp \left(-\sum_{l=2}^{\infty} F_{k}^{(l)} / 2 l\right) \\
& F_{k}^{(l)}=\sum_{v_{1}, \ldots, v_{l}=1}^{k} F_{k v_{1} \cdots v_{l}}^{(l)}, \\
& F_{k v_{1} \cdots v_{l}}^{(l)}=\int \cdots \int \prod_{j=1}^{l}\left\{\frac{d \theta_{j}}{2 \pi}\left(-\varepsilon_{v_{j} v_{j+1}}\right) e^{-i \varepsilon_{v_{j} v_{j+1}}\left(\left(m_{v_{j}}-m_{v_{j}+1}\right) \theta_{J}-\left(n_{v_{j}}-n_{v_{j+1}}\right) E_{j}\right)}\right. \\
& \left.\quad \times R^{\varepsilon_{v_{j-1} v_{j}},-\varepsilon_{v_{j} v_{j}+1}}\left(\theta_{j-1}, \theta_{j}\right)\right\}
\end{aligned}
$$

where $E_{j}=E\left(\theta_{j}\right)$ and $\varepsilon_{\mu v}=1(\mu>v),=0(\mu=v),=-1(\mu<v)$,

$$
\begin{aligned}
& \left\langle g_{m_{1} n_{1}} \cdots g_{m_{k_{1}} n_{k_{1}}}^{(1)} \cdots g_{m_{k_{2}} n_{k_{2}}}^{(2)} \cdots g_{m_{k} n_{k}}\right\rangle /\left\langle g_{m_{1} n_{1}} \cdots g_{m_{k} n_{k}}\right\rangle=\sum_{l=0}^{\infty} c_{k_{1} k_{2}}^{(l)}, \\
& c_{k_{1} k_{2}}^{(l)}=\sum_{v_{1}, \ldots, v_{l}=1}^{k} c_{k_{1} k_{2} v_{1} \cdots v_{l}}^{(l)} \text {, } \\
& c_{k_{1} k_{2} v_{1} \cdots v_{l}}^{(l)}=\int \cdots \int \prod_{j=0}^{l}\left(\underline{d \theta}_{j} e^{-i \varepsilon_{v_{J} v_{j+1}}\left(\left(m_{v_{j}}-m_{v_{j}+1}\right) \theta_{J}-\left(n_{v_{j}}-n_{v_{j+1}}\right) E_{j}\right)}\right) \\
& \times c_{1}^{-\varepsilon_{\mu v_{1}}}\left(\theta_{0}\right)\left(-\varepsilon_{\mu v_{1}}\right) R^{\varepsilon_{\mu v_{1}},-\varepsilon_{v_{1} v_{2}}}\left(\theta_{0}, \theta_{1}\right)\left(-\varepsilon_{v_{1} v_{2}}\right) \\
& \times R^{\varepsilon_{v_{1} v_{2}},-\varepsilon_{v_{2} v_{3}}}\left(\theta_{1}, \theta_{2}\right) \times \cdots \times\left(-\varepsilon_{v_{l-1} v_{l}}\right) R^{\varepsilon_{v_{l-1} v_{l}},-\varepsilon_{v_{l}} k_{2}}\left(\theta_{l-1}, \theta_{l}\right) \\
& \times\left(-\varepsilon_{v_{l} k_{2}}\right) c_{2}^{\varepsilon_{v} k_{2}}\left(\theta_{l}\right) \quad\left(v_{0}=k_{1}, v_{l+1}=k_{2}\right)
\end{aligned}
$$

where $k_{1} \leqq k_{2}$, and if $k_{1}=k_{2}$ the left hand side means $\left\langle g_{m_{1} n_{1}} \cdots g_{m_{k_{1}} n_{k_{1}}}^{\prime \prime} \cdots g_{m_{k} n_{k}}\right\rangle$. In general an arbitrary $k$-point function involving $g_{m n}, g_{m n}^{(j) \prime}$ and $g_{m n}^{\prime \prime}$ is expressed by using a Pfaffian with entries (5.6.17). See formula (1.4.10) in [1]. For instance we have

$$
\begin{aligned}
& \left\langle g_{m_{1} n_{1}} \cdots g_{m_{k_{1}} n_{k_{1}}} \cdots g_{m_{k_{2}} n_{k_{2}}}^{\prime \prime} \cdots g_{m_{k} n_{k}}\right\rangle \cdot\left\langle g_{m_{1} n_{1}} \cdots g_{m_{k} n_{k}}\right\rangle \\
= & \left\langle g_{m_{1} n_{1}} \cdots g_{m_{k_{1}} n_{k_{1}}}^{\prime \prime} \cdots g_{m_{k} n_{k}}\right\rangle \cdot\left\langle g_{m_{1} n_{1}} \cdots g_{m_{k_{2}} n_{k_{2}}}^{\prime \prime} \cdots g_{m_{k} n_{k}}\right\rangle \\
& -\left\langle g_{m_{1} n_{1}} \cdots g_{m_{k_{1}} n_{k_{1}}}^{(1)} \cdots g_{m_{k_{2}} n_{k_{2}}}^{(2)} \cdots g_{m_{k} n_{k}}\right\rangle \cdot\left\langle g_{m_{1} n_{1}} \cdots g_{m_{k_{1}} n_{k_{1}}}^{(2)} \cdots g_{m_{k_{2}} n_{k_{2}}}^{(1)} \cdots g_{m_{k} n_{k}}\right\rangle
\end{aligned}
$$




$$
+\left\langle g_{m_{1} n_{1}} \cdots g_{m_{k_{1}} n_{k_{1}}}^{(1)} \cdots g_{m_{k_{2}} n_{k_{2}}}^{(1)}, g_{m_{k} n_{k}}\right\rangle \cdot\left\langle g_{m_{1} n_{1}} \cdots g_{m_{k_{1}} n_{k_{1}}}^{(2),} \cdots g_{m_{k_{2}} n_{k_{2}}}^{(2)} \cdots g_{m_{k} n_{k}}^{\prime}\right\rangle
$$

and so forth.

Let us now compute the scaling limit of spin operators near the critical points, i.e. the singularities of the energy $E(\theta)$. There are three possibilities:

(i) $\pm \quad \theta \rightarrow 0, h \rightarrow 1 \pm 0, \gamma>0$ fixed.

(ii) $\gamma \rightarrow 0, \theta \rightarrow \pm \theta_{0}$ with $h=\cos \theta_{0},|h|<1$.

(iii) $\pm \quad \theta \rightarrow \pi, h \rightarrow-1 \mp 0, \gamma>0$ fixed.

Case (i) $)_{+}$. We set

$$
h=1+\mu \gamma \varepsilon, \quad \theta=\varepsilon p^{1}, \quad x^{0}=n \gamma \varepsilon, \quad x^{1}=m \varepsilon
$$

and take the limit $\varepsilon \rightarrow 0, m, n \rightarrow \infty$ under fixed $\gamma, p^{1}, x^{0}$ and $x^{1}$. Here $\mu>0$ is an arbitrarily chosen constant. We have then

$$
\begin{aligned}
& E(\theta)=\varepsilon \gamma \omega\left(p^{1}\right)+\cdots, \omega(p)=\sqrt{u^{2}+p^{2}} \\
& b_{1}(\theta)=\sqrt{\varepsilon} \sqrt{\frac{1+\gamma}{2 \gamma}} \sqrt{\mu-i p^{1}}+\cdots \\
& \alpha_{+}=\frac{1+\gamma}{1-\gamma}(1+\mu \varepsilon+\cdots), \alpha_{-}=1-\mu \varepsilon+\cdots
\end{aligned}
$$

As in (5.5.8) we introduce $\psi(u)=\sqrt{\varepsilon \omega\left(p^{1}\right)} \hat{\psi}_{1}(\theta)(u>0),=\sqrt{\varepsilon \omega\left(p^{1}\right)} \hat{\psi}_{1}^{\dagger}(-\theta)$ $(u<0)$ where $u^{ \pm 1}=\left(\varepsilon(u) \omega\left(p^{1}\right) \pm p^{1}\right) / \mu$. The result reads as follows :

$$
\begin{aligned}
\operatorname{Nr}\left(\sigma_{m n}^{x}\right) & =\sqrt{\frac{2 \gamma}{1+\gamma}}\left(\frac{2 \mu \varepsilon}{\gamma}\right)^{1 / 8} \operatorname{Nr}\left(\varphi^{F}(x)\right)+o\left(\varepsilon^{1 / 8}\right) \\
\operatorname{Nr}\left(i \sigma_{m n}^{y}\right) & =\sqrt{\frac{1+\gamma}{2 \gamma}}\left(\frac{2 \mu}{\gamma}\right)^{1 / 8} \varepsilon^{9 / 8} i \frac{\partial \psi_{0}}{\partial x^{0}}(x) e^{\rho_{F}(x) / 2}+o\left(\varepsilon^{9 / 8}\right) \\
& =\sqrt{\frac{1+\gamma}{2 \gamma}}\left(\frac{2 \mu}{\gamma}\right)^{1 / 8} \varepsilon^{9 / 8} \operatorname{Nr}\left(i \frac{\partial \varphi^{F}}{\partial x^{0}}(x)\right)+o\left(\varepsilon^{9 / 8}\right) .
\end{aligned}
$$

Here $\psi_{0}(x), \rho_{F}(x)$ and $\varphi^{F}(x)$ are given in (5.5.13).

The third equality of (5.6.20) follows from the fact that $\psi_{0}(x) \frac{\partial \rho_{F}}{\partial x^{ \pm}}(x)=0$ (see (4.3.79), (4.3.80)).

Case (i)_. Set $h=1-\mu \gamma \varepsilon$ and define $p^{1}, x^{0}, x^{1}$ as in (5.6.18). The leading behavior of $E(\theta)$ and $\alpha_{+}$are given by (5.6.19) $)_{+}$, while $\alpha_{-}^{-1}=1-\mu \varepsilon+\cdots$ and $b_{1}(\theta)$ is replaced by

$$
b_{2}(\theta)=\frac{1}{\sqrt{\varepsilon}} \sqrt{\frac{1+\gamma}{2 \gamma}} \frac{1}{\sqrt{\mu-i p^{1}}}+\cdots .
$$

In this case we modify the definition of $\psi(u)$ as $\psi(u)=-i \sqrt{\varepsilon \omega\left(p^{1}\right)} \hat{\psi}_{2}(\theta)(u>0)$, $=i \sqrt{\varepsilon \omega\left(p^{1}\right)} \hat{\psi}_{2}(-\theta)(u<0)$. Noting the fact that $\psi_{2, m n}^{y} \psi_{2, m-1, n}^{y}=-\psi_{2, m n}^{y}\left(\psi_{2, m n}^{y}\right.$ 
$\left.-\psi_{2, m-1, n}^{y}\right)=\varepsilon \frac{1+\gamma}{2 \gamma} \psi_{0}(x) \cdot \frac{\partial \psi_{0}}{\partial x^{1}}(x)+\cdots$, we obtain

$$
\begin{aligned}
\operatorname{Nr}\left(\bar{\sigma}_{m n}^{x}\right) & =\sqrt{\frac{2 \gamma}{1+\gamma}}\left(\frac{2 \mu \varepsilon}{\gamma}\right)^{1 / 8} \operatorname{Nr}\left(\varphi_{F}(x)\right)+o\left(\varepsilon^{1 / 8}\right) \\
\operatorname{Nr}\left(i \bar{\sigma}_{m n}^{y}\right) & =\sqrt{\frac{1+\gamma}{2 \gamma}}\left(\frac{2 \mu}{\gamma}\right)^{1 / 8} \varepsilon^{9 / 8} \psi_{0}(x) \frac{\partial \psi_{0}}{\partial x^{1}}(x) e^{\rho_{F}(x) / 2}+o\left(\varepsilon^{9 / 8}\right) \\
& =\sqrt{\frac{1+\gamma}{2 \gamma}}\left(\frac{2 \mu}{\gamma}\right)^{1 / 8} \varepsilon^{9 / 8} \operatorname{Nr}\left(i \frac{\partial \varphi_{F}}{\partial x^{0}}(x)\right)+o\left(\varepsilon^{9 / 8}\right),
\end{aligned}
$$

where $\varphi_{F}(x)$ is given in (5.5.21).

Case (ii). Here we set

$$
\gamma=\mu \varepsilon, \quad x^{0}=n \varepsilon\left|\sin \theta_{0}\right|, \quad x^{1}=m \varepsilon
$$

and $\theta= \pm \theta_{0}+\varepsilon p^{1}$ in the region $\theta \sim \pm \theta_{0}$. We have

$$
\begin{aligned}
& E(\theta)=\varepsilon\left|\sin \theta_{0}\right| \omega\left(p^{1}\right)+\cdots \\
& \alpha_{ \pm}=e^{ \pm i \theta_{0}}(1+\mu \varepsilon)+\cdots \\
& b_{2}(\theta)=\varepsilon\left(1-e^{ \pm 2 i \theta_{0}}\right)\left(\mu-i p^{1}\right)
\end{aligned}
$$

according as $\theta \rightarrow \pm \theta_{0}$. Consider first $\rho_{2, m n}$ appearing in (5.6.12) $\mathscr{\mathscr { R }}_{2}$. In the limit $m, n \rightarrow \infty, \varepsilon \rightarrow 0$, the only contributions come from the regions $\sigma \theta+\sigma^{\prime} \theta^{\prime}=0$ $\left(\sigma, \sigma^{\prime}= \pm\right), \theta, \theta^{\prime}=\theta_{0}$ or $-\theta_{0}$, due to the rapid oscillation of the exponential factors in (5.2.18) (where $b(\theta)$ and $\gamma(\theta)$ are replaced by $b_{2}(\theta)$ and $-i E(\theta)$, respectively). Writing

$$
\hat{\psi}^{(1,2) \dagger}\left(p^{1}\right)=\sqrt{\varepsilon \omega\left(p^{1}\right)} \hat{\psi}^{\dagger}\left( \pm \theta_{0}+\varepsilon p^{1}\right), \quad \hat{\psi}^{(1,2)}\left(p^{1}\right)=\sqrt{\varepsilon \omega\left(p^{1}\right)} \hat{\psi}\left( \pm \theta_{0}+\varepsilon p^{1}\right),
$$

we get

$$
\begin{aligned}
& \rho_{2, m n}=2 \iint_{-\infty}^{+\infty} \frac{d p^{1}}{2 \pi \omega} \frac{d p^{1^{\prime}}}{2 \pi \omega^{\prime}}\left\{\hat{\psi}^{(1) \dagger}\left(p^{1}\right) \hat{\psi}^{(2) \dagger}\left(p^{\left.1^{\prime}\right)}\right) \frac{i\left(\omega-\omega^{\prime}\right)}{p^{1}+p^{1^{\prime}}+i 0} e^{i x^{0}\left(\omega+\omega^{\prime}\right)-i x^{1}\left(p^{\left.1+p^{1}\right)}\right)}\right. \\
& +\hat{\psi}^{(1)}\left(p^{1}\right) \hat{\psi}^{(2)}\left(p^{1^{\prime}}\right) \frac{-i\left(-\omega+\omega^{\prime}\right)}{p^{1}+p^{1^{\prime}}-i 0} e^{-i x^{0}\left(\omega+\omega^{\prime}\right)+i x^{1}\left(p^{1+p^{1}}\right)}+\left(\hat{\psi}^{(1)}\left(p^{1}\right) \hat{\psi}^{(1) \dagger}\left(p^{1^{\prime}}\right)\right. \\
& \left.\left.\quad+\hat{\psi}^{(2)}\left(p^{1}\right) \hat{\psi}^{(2) \dagger}\left(p^{1^{\prime}}\right)\right) \frac{-i\left(\omega+\omega^{\prime}\right)}{p^{1}-p^{1^{\prime}}-i 0} e^{-i x^{0}\left(\omega-\omega^{\prime}\right)+i x^{1}\left(p^{1}-p^{1}\right)}\right\}+\cdots
\end{aligned}
$$

with $\omega=\omega\left(p^{1}\right), \omega^{\prime}=\omega\left(p^{1^{\prime}}\right)$. Making use of the canonical transformation

$$
\begin{aligned}
& \sqrt{2} \hat{\psi}^{(1) \dagger}\left(p^{1}\right)=i \psi^{(1) \dagger}\left(p^{1}\right)+\psi^{(2) \dagger}\left(p^{1}\right) \\
& \sqrt{2} \hat{\psi}^{(2) \dagger}\left(p^{1}\right)=i \psi^{(1) \dagger}\left(p^{1}\right)-\psi^{(2) \dagger}\left(p^{1}\right) \\
& \sqrt{2} \hat{\psi}^{(1)}\left(p^{1}\right)=-i \psi^{(1)}\left(p^{1}\right)+\psi^{(2)}\left(p^{1}\right) \\
& \sqrt{2} \hat{\psi}^{(2)}\left(p^{1}\right)=-i \psi^{(1)}\left(p^{1}\right)-\psi^{(2)}\left(p^{1}\right)
\end{aligned}
$$


and setting $\psi^{(j)}(u)=\psi^{(j)}\left(p^{1}\right)(u>0),=\psi^{(j)^{\dagger}}\left(-p^{1}\right)(u<0)$ we have

$$
\rho_{2, m n}=\rho_{F}^{(1)}(x)+\rho_{F}^{(2)}(x)+o(1) .
$$

Here $\rho_{F}^{(j)}(x)$ is obtained from (5.5.21) by replacing $\psi(u)$ by mutually independent free fermion operators $\psi^{(j)}(u)(j=1,2)$. The scaled form for $\psi_{2, m n}^{y} \psi_{2, m-1, n}^{y}$ is calculated similarly. Thus we find

$$
\begin{aligned}
& \operatorname{Nr}\left(\bar{\sigma}_{m n}^{x}\right)=\left(4 \sqrt{1-h^{2}} \mu \varepsilon\right)^{1 / 4} \operatorname{Nr}\left(\varphi_{F}^{(1)}(x) \otimes \varphi_{F}^{(2)}(x)\right)+o\left(\varepsilon^{1 / 4}\right) \\
& \operatorname{Nr}\left(i \bar{\sigma}_{m n}^{y}\right)=-\left(4 \sqrt{1-h^{2}} \mu \varepsilon\right)^{1 / 4} \operatorname{Nr}\left(\varphi^{F(1)}(x) \otimes \varphi^{F(2)}(x)\right)+o\left(\varepsilon^{1 / 4}\right) .
\end{aligned}
$$

Since the scaled spin operators are the tensor product $\varphi_{F}^{(1)}(x) \otimes \varphi_{F}^{(2)}(x)$ or $\varphi^{F^{(1)}}(x)$ $\otimes \varphi^{F(2)}(x)$ of copies of identical ones, their $n$-point functions coincide with the squares of those for the Ising model. (For the 2-point function this result was obtained in [13].)

Case (iii) $)_{+}$Setting $h=-1-\mu \gamma \varepsilon, \theta=\pi+\varepsilon p^{1}$ we see that $(5.6 .19)_{+}$holds, where $\alpha_{ \pm}$should be replaced by $-\alpha_{\mp}$. Therefore (5.6.13) $)_{\mathscr{R}_{3}}$ implies

$$
\begin{aligned}
& \operatorname{Nr}\left((-)^{m} \sigma_{m n}^{x}\right)=\sqrt{\frac{2 \gamma}{1+\gamma}}\left(\frac{2 \mu \varepsilon}{\gamma}\right)^{1 / 8} \operatorname{Nr}\left(\varphi^{F}(x)\right)+o\left(\varepsilon^{1 / 8}\right) \\
& \operatorname{Nr}\left((-)^{m+1} i \sigma_{m n}^{y}\right)=\sqrt{\frac{1+\gamma}{2 \gamma}}\left(\frac{2 \mu}{\gamma}\right)^{1 / 8} \varepsilon^{9 / 8} \operatorname{Nr}\left(i \frac{\partial \varphi^{F}}{\partial x^{0}}(x)\right)+o\left(\varepsilon^{9 / 8}\right) .
\end{aligned}
$$

Case (iii)_. In this case $h=-1+\mu \gamma \varepsilon, \theta=\pi+\varepsilon p^{1},-\alpha_{+}=1-\mu \varepsilon+\cdots$ and $-\alpha_{-}=(1+\gamma) /(1-\gamma)$. (5.6.19) $)_{-}$holds without any change. Hence we have

$$
\begin{aligned}
& \operatorname{Nr}\left((-)^{m} \sigma_{m n}^{x}\right)=\sqrt{\frac{2 \gamma}{1+\gamma}}\left(\frac{2 \mu \varepsilon}{\gamma}\right)^{1 / 8} \operatorname{Nr}\left(\varphi_{F}(x)\right)+o\left(\varepsilon^{1 / 8}\right) \\
& \operatorname{Nr}\left((-)^{m+1} i \sigma_{m n}^{y}\right)=-\sqrt{\frac{1+\gamma}{2 \gamma}}\left(\frac{2 \mu}{\gamma}\right)^{1 / 8} \varepsilon^{9 / 8} \operatorname{Nr}\left(i \frac{\partial \varphi_{F}}{\partial x^{0}}(x)\right)+o\left(\varepsilon^{9 / 8}\right) .
\end{aligned}
$$

\section{§5.7. The Orthogonal Model}

In this section we formulate a general orthogonal version of lattice models ([13], [14], [15]) using the Grassmann integral and solve it analogously as in Section 5.4 .

First we prepare some generalities on the Grassmann integral. Let $W$ be an $N$-dimensional vector space, and let $\omega$ be a non-zero element of $\Lambda^{N}(W)$. The Grassmann integral with respect to $\omega$ is a linear form on $\Lambda(W)$, 


$$
\begin{aligned}
\int \omega^{-1}: & \Lambda(w) \\
\Psi & \longrightarrow \mathbb{C} \\
& a \longmapsto \int \omega^{-1} a
\end{aligned}
$$

such that the map $a \mapsto \omega\left(\int \omega^{-1} a\right)$ coincides with the projection onto $\Lambda^{N}(W)$.

If $\omega=c \omega^{\prime}$ for some $c \in \mathbb{C}$, we have

$$
\int \omega^{-1} a=c^{-1} \int \omega^{\prime-1} a \quad(c \neq 0) .
$$

Let $W^{\prime}$ be another vector space of dimensions $N^{\prime}$. For $\tilde{a} \in \Lambda\left(W \oplus W^{\prime}\right)$ we also define $\int \omega^{-1} \tilde{a} \in \Lambda\left(W^{\prime}\right)$ so that $\tilde{a} \mapsto \omega_{\wedge}\left(\int \omega^{-1} \tilde{a}\right)$ coincides with the projection $\Lambda\left(W \oplus W^{\prime}\right) \rightarrow \Lambda^{N}(W)_{\wedge} \Lambda\left(W^{\prime}\right)$. If $\omega^{\prime} \in \Lambda^{N^{\prime}}\left(W^{\prime}\right)$, we have

$$
\int \omega^{\prime-1} \int \omega^{-1} \tilde{a}=\int\left(\omega \omega^{\prime}\right)^{-1} \tilde{a}
$$

Let $v_{1}, \ldots, v_{N}$ be a basis of $W$, and set $\omega=v_{1} \cdots v_{N}$. For an anti-symmetric matrix $F=\left(f_{j k}\right)_{j, k=1, \ldots, N}$, we set $S=\frac{1}{2} \sum_{j, k=1}^{N} f_{j k} v_{j} v_{k} \in \Lambda^{2}(W)$. Then we have

$$
\begin{aligned}
& \int \omega^{-1} e^{s}=\text { Pfaffian } F, \\
& \left(\int \omega^{-1} e^{s} v_{j} v_{k} / \int \omega^{-1} e^{s}\right)_{j, k=1, \ldots, N}=-F^{-1} .
\end{aligned}
$$

In general, for $w_{1}, \ldots, w_{s} \in W$ we have

$$
\int \omega^{-1} e^{s} w_{1} \cdots u_{s} / \int \omega^{-1} e^{s}=\text { Pfaffian }\left(h_{j k}\right)_{j, k=1, \ldots, s}
$$

where $h_{j k}=\int \omega^{-1} e^{S} w_{j} w_{k} / \int \omega^{-1} e^{S}$.

Consider a rectangular lattice $L$ of size $M \times N$ with cyclic boundary and with even $M$ and $N$. To each site $(m, n)$ we attach a 4-dimensional vector space $\mathscr{W}_{m n}=\boldsymbol{C} u_{m n} \oplus \boldsymbol{C} v_{m n} \oplus \boldsymbol{C} u_{m n}^{\dagger} \oplus \boldsymbol{C} v_{m n}^{+}$, and set $\mathscr{W}=\sum_{m=0}^{M-1} \sum_{n=0}^{N-1} \mathscr{W}_{m n}$.

We set in $\Lambda(\mathscr{W})$

$$
\begin{aligned}
\omega_{\mathscr{F}}= & \prod_{m=0}^{M-1} \prod_{n=0}^{N-1} v_{m n}^{\dagger} v_{m n} u_{m n}^{\dagger} u_{m n}, \\
\mathscr{S}^{(0)}= & \sum_{m=0}^{M-1} \sum_{n=0}^{N-1}\left(f_{12} u_{m n} v_{m n}+f_{13} u_{m n} u_{m n}^{\dagger}+f_{14} u_{m n} v_{m n}^{\dagger}+f_{23} v_{m n} u_{m n}^{\dagger}\right. \\
& \left.+f_{24} v_{m n} v_{m n}^{\dagger}+f_{34} u_{m n}^{\dagger} v_{m n}^{\dagger}\right), \\
\mathscr{S}^{(1)}= & \sum_{m=0}^{M-1} \sum_{n=0}^{N-1} u_{m n} u_{m+1, n}^{\dagger},
\end{aligned}
$$




$$
\mathscr{S}^{(2)}=\sum_{m=0}^{M-1} \sum_{n=0}^{N-1} v_{m n} v_{m, n+1}^{\dagger} .
$$

The grand partition function is defined by the integral (see (5.4.2))

$$
Z_{M N}=\int \omega^{-1} e^{\mathscr{S}(0)+\mathscr{S}(1)+\mathscr{S}(2)} \text {. }
$$

In order to compute $Z_{M N}$ we define the Fourier transformation by

$$
\begin{gathered}
\left(\begin{array}{c}
\hat{u}\left(\theta_{\mu}, \theta_{v}^{\prime}\right) \\
\hat{v}\left(\theta_{\mu}, \theta_{v}^{\prime}\right)
\end{array}\right)=\sum_{m=0}^{M-1} \sum_{n=0}^{N-1} e^{-i m \theta_{\mu}-i n \theta_{v}^{\prime}}\left(\begin{array}{c}
u_{m n} \\
v_{m n}
\end{array}\right), \\
\left(\begin{array}{c}
\hat{u}^{\dagger}\left(\theta_{\mu}, \theta_{v}^{\prime}\right) \\
\hat{v}^{\dagger}\left(\theta_{\mu}, \theta_{v}^{\prime}\right)
\end{array}\right)=\sum_{m=0}^{M-1} \sum_{n=0}^{N-1} e^{i m \theta_{\mu}+i n \theta_{v}^{\prime}}\left(\begin{array}{c}
u_{m n}^{\dagger} \\
v_{m n}^{\dagger}
\end{array}\right), \\
\left(\theta_{\mu}=\frac{2 \pi \mu}{M}, \theta_{v}^{\prime}=\frac{2 \pi v}{N} ; \mu=\frac{-M+1}{2}, \ldots, \frac{M-1}{2}\right. \\
\left.v=\frac{-N+1}{2}, \ldots, \frac{N-1}{2}\right) .
\end{gathered}
$$

Then we have

$$
\begin{aligned}
& \mathscr{S}^{(0)}+\mathscr{S}^{(1)}+\mathscr{S}^{(2)} \\
= & \frac{1}{M N} \sum_{\mu=(-M+1) / 2} \sum_{v=(-N+1) / 2}^{(N-1) / 2}\left(f_{12} \hat{u}\left(\theta_{\mu}, \theta_{v}^{\prime}\right) \hat{v}\left(-\theta_{\mu},-\theta_{v}^{\prime}\right)\right. \\
& +\left(f_{13}+e^{-i \theta_{\mu}}\right) \hat{u}\left(\theta_{\mu}, \theta_{v}^{\prime}\right) \hat{u}^{\dagger}\left(\theta_{\mu}, \theta_{v}^{\prime}\right)+f_{14} \hat{u}\left(\theta_{\mu}, \theta_{v}^{\prime}\right) \hat{v}^{\dagger}\left(\theta_{\mu}, \theta_{v}^{\prime}\right) \\
& +f_{23} \hat{v}\left(\theta_{\mu}, \theta_{v}^{\prime}\right) \hat{u}^{\dagger}\left(\theta_{\mu}, \theta_{v}^{\prime}\right)+\left(f_{24}+e^{-i \theta_{v}^{\prime}}\right) \hat{v}\left(\theta_{\mu}, \theta_{v}^{\prime}\right) \hat{v}^{\dagger}\left(\theta_{\mu}, \theta_{v}^{\prime}\right) \\
& \left.+f_{34} \hat{u}^{\dagger}\left(\theta_{\mu}, \theta_{v}^{\prime}\right) \hat{v}^{\dagger}\left(-\theta_{\mu},-\theta_{v}^{\prime}\right)\right) .
\end{aligned}
$$

From (5.7.2) and (5.7.4) we have ([14], [15])

$$
\begin{aligned}
Z_{M N} & =\prod_{\mu=(-M+1) / 2}^{(M-1) / 2} \prod_{v=(-N+1) / 2}^{(N-1) / 2} \\
& \operatorname{det}\left(\begin{array}{llll}
0 & f_{12} & f_{13}+e^{-i \theta_{\mu}} & f_{14} \\
-f_{12} & 0 & f_{23} & f_{24}+e^{-i \theta_{v}^{\prime}} \\
-f_{13}-e^{i \theta_{\mu}} & -f_{23} & 0 & f_{34} \\
-f_{14} & -f_{24}-e^{i \theta_{v}^{\prime}} & -f_{34} & 0
\end{array}\right) \\
& =\prod_{\mu=(-M+1) / 2} \prod_{v=(-N+1) / 2}^{(N-1) / 2} \Delta\left(\theta_{\mu}, \theta_{v}^{\prime}\right)
\end{aligned}
$$

where $\Delta\left(\theta, \theta^{\prime}\right)=1+f_{1234}^{2}+f_{13}^{2}+f_{24}^{2}-2 \cos \theta\left(f_{1234} f_{24}-f_{13}\right)-2 \cos \theta^{\prime}\left(f_{1234} f_{13}\right.$ $\left.-f_{24}\right)-2 \cos \left(\theta-\theta^{\prime}\right)\left(f_{12} f_{34}-f_{13} f_{24}\right)-2 \cos \left(\theta+\theta^{\prime}\right)\left(f_{14} f_{23}-f_{13} f_{24}\right)$ with

$$
f_{1234}=f_{12} f_{34}-f_{13} f_{24}+f_{14} f_{23} .
$$

For a 1-chain $\Gamma$ we set (cf. p. 557) 


$$
\begin{aligned}
& \mathscr{S}^{(1)}(\Gamma)=\sum_{m=0}^{M-1} \sum_{n=0}^{N-1} \varepsilon_{m n}^{(1)}(\Gamma) u_{m n} u_{m+1, n}^{\dagger}, \\
& \mathscr{S}^{(2)}(\Gamma)=\sum_{m=0}^{M-1} \sum_{n=0}^{N-1} \varepsilon_{m n}^{(2)}(\Gamma) v_{m n} v_{m, n+1}^{\dagger} .
\end{aligned}
$$

The correlation function for $\partial \Gamma$ is defined by

$$
\rho(\partial \Gamma)=Z_{M N}^{-1} \int \omega_{\mathscr{Y}}^{-1} e^{\mathscr{P}(0)+\mathscr{S}(1)(\Gamma)+\mathscr{S}(2)(\Gamma)} .
$$

$\rho(\partial \Gamma)$ depends only on $\partial \Gamma$ (see Proposition 5.4.1). In order to compute $\rho(\partial \Gamma)$ we use the transfer matrix formalism.

We consider $2 M$-dimensional vector spaces $W=\sum_{m=0}^{M-1} \mathbb{C} v_{m}^{\dagger} \oplus \sum_{m=0}^{M-1} \mathbb{C} v_{m}$ and $W^{\prime}=\sum_{m=0}^{M-1} \boldsymbol{C} u_{m}^{\dagger}+\sum_{m=0}^{M-1} \mathbf{C} u_{m} . \quad$ We set in $\Lambda\left(W \oplus W^{\prime}\right)$

$$
\begin{aligned}
& \omega_{W}=\prod_{m=0}^{M-1} v_{m}^{\dagger} v_{m}, \quad \omega_{W^{\prime}}=\prod_{m=0}^{M-1} u_{m}^{\dagger} u_{m}, \\
& S_{0}=\sum_{m=0}^{M-1}\left(f_{12} u_{m} v_{m}+f_{13} u_{m} u_{m}^{\dagger}+f_{14} u_{m} v_{m}^{\dagger}+f_{23} v_{m} u_{m}^{\dagger}+f_{24} v_{m} v_{m}^{\dagger}\right. \\
& \left.+f_{34} u_{m}^{\dagger} v_{m}^{\dagger}\right) \\
& S_{1}=\sum_{m=0}^{M-1} u_{m} u_{m+1}^{\dagger} \text {. }
\end{aligned}
$$

We equip $W$ with an orthogonal structure by the inner product $\langle$,$\rangle such that$ $\left\langle v_{m}^{\dagger}, v_{m^{\prime}}^{\dagger}\right\rangle=0,\left\langle v_{m}, v_{m^{\prime}}\right\rangle=0$ and $\left\langle v_{m}^{\dagger}, v_{m^{\prime}}\right\rangle=\delta_{m m^{\prime}}$. We denote by $\langle\mathrm{vac}|$ and $|v a c\rangle$ the vacuums with respect to the holonomic decomposition $W=W_{\text {cre }} \oplus$ $W_{a n n}$ where $W_{c r e}=\sum_{m=0}^{M-1} \boldsymbol{C} v_{m}^{\dagger}$ and $W_{a n n}=\sum_{m=0}^{M-1} \mathbb{C} v_{m}$. We also denote by $\left\langle m_{1} \cdots m_{k}\right|$ (resp. $\left.\left|m_{1} \cdots m_{k}\right\rangle\right)$ the state vector $\langle\operatorname{vac}| v_{m_{1}} \cdots v_{m_{k}}$ (resp. $v_{m_{1}}^{\dagger} \cdots v_{m_{k}}^{\dagger}|\mathrm{vac}\rangle$ ).

We define an element $V$ of $A(W)$ by specifying its matrix elements as follows.

$$
\begin{aligned}
& \left\langle m_{1} \cdots m_{j}|V| m_{k}^{\prime} \cdots m_{1}^{\prime}\right\rangle \\
= & \int \omega_{W}^{-1} \int \omega_{W^{\prime}}^{-1} e^{S_{0}+S_{1}} v_{m_{1}}^{\dagger} \cdots v_{m_{j}}^{\dagger} v_{m_{k}^{\prime}} \cdots v_{m_{1}^{\prime}}
\end{aligned}
$$

Then we have

$$
Z_{M N}=\operatorname{trace} V^{N}
$$

Thus $V$ is the transfer matrix of our system.

Proposition 5.7.1. V belongs to the Clifford group $G(W)$.

Proof. By Theorem 1.4.4 an element $g \in A(W)$ such that $\langle g\rangle=1$ belongs to $G(W)$ if and only if the matrix elements $\left\langle m_{1} \cdots m_{j}|g| m_{k}^{\prime} \cdots m_{1}^{\prime}\right\rangle$ satisfy the 
condition

$$
\begin{aligned}
& \left\langle m_{1} \cdots m_{j}|g| m_{k}^{\prime} \cdots m_{1}^{\prime}\right\rangle=\text { Pfaffian }
\end{aligned}
$$

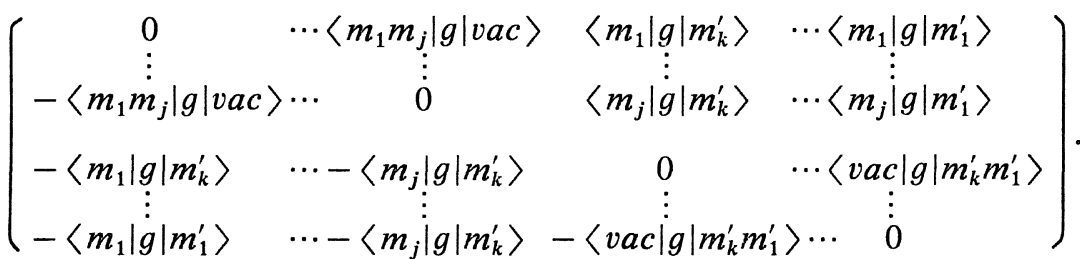

Hence the proposition follows from (5.7.6).

Now we define the spin operator $s_{m}$ by

$$
s_{m}=\prod_{j=0}^{m-1}\left(1-2 v_{m}^{\dagger} v_{m}\right)
$$

If we set

$$
p_{m}=v_{m}^{\dagger}+v_{m}, \quad q_{m}=-v_{m}^{\dagger}+v_{m},
$$

we have

$$
s_{m}=q_{m-1} p_{m-1} \cdots q_{0} p_{0} .
$$

$s_{m}$ belongs to $G(W)$ and the induced rotation is given by

$$
\begin{aligned}
& T_{s_{m}} v_{j}^{\dagger}= \begin{cases}v_{j}^{\dagger} & j \geqq m \\
-v_{j}^{\dagger} & j \leqq m-1,\end{cases} \\
& T_{s_{m}} v_{j}= \begin{cases}v_{j} & j \geqq m \\
-v_{j} & j \leqq m-1 .\end{cases}
\end{aligned}
$$

\section{Proposition 5.7.2.}

$$
\begin{aligned}
& \rho\left(\left(m_{1}, n_{1}\right), \ldots,\left(m_{k}, n_{k}\right)\right) \\
= & Z_{M N}^{-1} \operatorname{trace} V^{n_{1}} s_{m_{1}} V^{n_{2}-n_{1}} s_{m_{2}} \cdots V^{n_{k}-n_{k-1}} s_{m_{h}} V^{N-n_{k}} .
\end{aligned}
$$

Proof. Note that

$$
\begin{aligned}
& \left\langle m_{1} \cdots m_{j}\left|s_{m} V\right| m_{k}^{\prime} \cdots m_{1}^{\prime}\right\rangle \\
= & \left\langle m_{1} \cdots m_{j}\left|s_{m}\right| m_{j} \cdots m_{1}\right\rangle\left\langle m_{1} \cdots m_{j}|V| m_{k}^{\prime} \cdots m_{1}^{\prime}\right\rangle \\
= & (-)^{\#\left\{j^{\prime} \mid m_{j^{\prime}} \leqq m-1\right\}}\left\langle m_{1} \cdots m_{j}|V| m_{k}^{\prime} \cdots m_{1}^{\prime}\right\rangle .
\end{aligned}
$$

Taking $\Gamma$ as the polygon in Figure 5.7.1 we can show the proposition. 


\begin{tabular}{|l|l|l|l|l|l|l|l|}
\hline & $\left(0, n_{k}\right)$ & & & & & & $\left(m_{k}, n_{k}\right)$ \\
\hline$x$ & & & & & & & \\
\hline & & & & & & $x$ & \\
\hline & $\left(0, n_{1}\right)$ & & & & $\left(m_{1}, n_{1}\right)$ & & \\
\hline$x$ & & & & $x$ & & & \\
\hline
\end{tabular}

Figure 5.7.1.

Now we shall diagonalize $V$. We define the Fourier transformation by

$$
\begin{aligned}
& \left(\begin{array}{l}
\hat{u}\left(\theta_{\mu}\right) \\
\hat{\imath}\left(\theta_{\mu}\right)
\end{array}\right)=\sum_{m=0}^{M-1} e^{-i m \theta_{\mu}}\left(\begin{array}{c}
u_{m} \\
v_{m}
\end{array}\right), \\
& \left(\begin{array}{l}
\hat{u}^{\dagger}\left(\theta_{\mu}\right) \\
\hat{v}^{\dagger}\left(\theta_{\mu}\right)
\end{array}\right)=\sum_{m=0}^{M-1} e^{i m \theta_{\mu}}\left(\begin{array}{c}
u_{m}^{\dagger} \\
v_{m}^{\dagger}
\end{array}\right) .
\end{aligned}
$$

Then we have

$$
\begin{aligned}
& e^{S_{\text {eff }}=}=\int \omega_{W^{-1}}^{-1} e^{S_{0}+S_{1}}=\prod_{\mu=(-M+1) / 2}^{(M-1) / 2} Q\left(\theta_{\mu}\right) \\
& Q(\theta)=\left(-f_{13}-e^{-i \theta}+\frac{1}{M}\left(f_{23} \hat{v}(\theta)-f_{34} \hat{v}^{\dagger}(-\theta)\right)\left(f_{12} \hat{v}(-\theta)\right.\right. \\
&\left.\left.+f_{14} \hat{v}^{\dagger}(\theta)\right)\right)\left(1+\frac{1}{M} f_{24} \hat{v}(\theta) \hat{v}^{\dagger}(\theta)\right)
\end{aligned}
$$

For simplicity sake we assume that $f_{12}=f_{34}$ and $f_{14}=f_{23}$. Since $\rho(\partial \Gamma)$ depends on $f_{12}$ and $f_{34}$ (resp. $f_{14}$ and $f_{23}$ ) through the product $f_{12} f_{34}$ (resp. $f_{14} f_{23}$ ), this is not a restriction. We set

$$
\begin{aligned}
& f_{12}=f_{34}=c, \\
& f_{14}=f_{23}=d, \\
& f_{13}=b_{+}, f_{24}=b_{-}, \\
& \text {Pfaffian } F=c^{2}+d^{2}-b_{+} b_{-}=a_{+} .
\end{aligned}
$$

Proposition 5.7.3. The induced rotation $T_{V}$ is given by

$$
\left(T_{V} \hat{v}^{\dagger}\left(-\theta_{\mu}\right), T_{V} \hat{v}\left(\theta_{\mu}\right)\right)=\left(\hat{v}^{\dagger}\left(-\theta_{\mu}\right), \hat{v}\left(\theta_{\mu}\right)\right) T\left(\theta_{\mu}\right)
$$

where

$$
\begin{aligned}
& T(\theta)=\left(\begin{array}{ll}
r(\theta) & 0 \\
s(\theta) & 1
\end{array}\right)^{-1}\left(\begin{array}{cc}
1 & s(\theta) \\
0 & r(-\theta)
\end{array}\right), \\
& r(\theta)=\frac{c^{2}}{b_{+}+e^{-i \theta}}+\frac{d^{2}}{b_{+}+e^{i \theta}}-b_{-}, \\
& s(\theta)=c d\left(\frac{1}{b_{+}+e^{-i \theta}}-\frac{1}{b_{+}+e^{i \theta}}\right) .
\end{aligned}
$$


Proof. From (5.7.25) and Proposition 5.7.1 we know that $V \hat{v}^{\dagger}\left(-\theta_{\mu}\right)$ and $V \hat{v}\left(-\theta_{\mu}\right)$ are linear combinations of $\hat{v}^{\dagger}\left(-\theta_{\mu}\right) V, \hat{v}\left(\theta_{\mu}\right) V, \hat{v}^{\dagger}\left(\theta_{\mu}\right) V$ and $\hat{v}\left(-\theta_{\mu}\right) V$. The coefficients are determined by computing the matrix elements of the forms

$$
\begin{aligned}
& \left\langle\theta_{(-M+1) / 2} \cdots \cdots \theta_{(M-1) / 2}|*| \theta_{(M-1) / 2} \cdots \theta_{(-M+1) / 2}\right\rangle \text {, and } \\
& \left\langle\theta_{(-M+1) / 2} \cdots \theta_{(M-1) / 2}|*| \theta_{(M-1) / 2} \cdots \cdots \theta_{(-M+1) / 2}\right\rangle \text {. }
\end{aligned}
$$

Using (5.7.20) we can rewrite (5.7.27) as

$$
\left(T_{V} \hat{p}\left(\theta_{\mu}\right), T_{V} \hat{q}\left(\theta_{\mu}\right)\right)=\left(\hat{p}\left(\theta_{\mu}\right), \hat{q}\left(\theta_{\mu}\right)\right) T^{\prime}\left(\theta_{\mu}\right)
$$

where

$$
T^{\prime}(\theta)=\frac{1}{B(\theta)+i C(\theta)}\left(\begin{array}{ll}
A(\theta) & e A_{+}(\theta) \\
e A_{-}(\theta) & A(\theta)
\end{array}\right) .
$$

Here $A_{ \pm}(\theta)$ (resp. $E(\theta)$ below) is given by (5.6.4) (resp. (5.6.5)) with

$$
\begin{aligned}
& h=\left(a_{+}^{2}+b_{-}^{2}-b_{+}^{2}-1\right) / e, \\
& \gamma=2 c d / e, \\
& e=a_{+} b_{-}+b_{+} .
\end{aligned}
$$

We have set also

$$
\begin{aligned}
A(\theta) & =\left(a_{+}^{2}+b_{-}^{2}+b_{+}^{2}+1\right) / 2-\left(a_{+} b_{-}-b_{+}\right) \cos \theta, \\
& =\sqrt{B(\theta)^{2}+C(\theta)^{2}+e^{2} E(\theta)^{2}}, \\
B(\theta) & =a_{+} b_{+}-b_{-}+\left(a_{+}-b_{+} b_{-}\right) \cos \theta, \\
C(\theta) & =\left(c^{2}-d^{2}\right) \sin \theta .
\end{aligned}
$$

(5.7.28) shows that the diagonalization of $V$ reduces to that of the $X Y$ model in Section 5.6. The renormalized transfer matrix $V_{\text {ren }}=\exp \left(-\mathscr{H}_{M, \text { ren }}\right)$ is given by (5.6.9) with $E\left(\theta_{\mu}\right)$ in $\mathscr{H}_{M \text {,ren }}$ replaced by

$$
\widetilde{E}\left(\theta_{\mu}\right)=\log \frac{A(\theta)+e E(\theta)}{B(\theta)+i C(\theta)} .
$$

Let us consider the expectation value $\langle a\rangle=Z_{M N}^{-1}$ trace $\left(a V^{N}\right)$. In the limit $M, N \rightarrow \infty, \hat{\psi}^{\dagger}(\theta)$ (resp. $\hat{\psi}(\theta)$ ) becomes the creation (resp. annihilation) operator. Let us compute the norm of $s_{m n}$ in this limit. From (5.7.21) and (5.7.28), this computation also reduces to that of the $X Y$ model. Namely in $\mathscr{R}_{1}\left(\right.$ resp. $\left.\mathscr{R}_{3}\right) \operatorname{Nr}\left(s_{m n}\right)$ of the orthogonal model is given by $\mathrm{Nr}\left(t_{m n}\right)\left(\operatorname{resp} . \operatorname{Nr}\left(t_{m n}^{\prime}\right)\right)$ with $E(\theta)$ replaced by $\tilde{E}(\theta)$. In $\mathscr{R}_{2}$ we must consider $\bar{s}_{m} \overline{\overline{\text { def }}} s_{m} p_{0}$ for the finite lattice and then take the limit. Then we have

$$
\operatorname{Nr}\left(\bar{s}_{m n}\right)=\psi_{2, m-1, n}^{y} \operatorname{Nr}\left(\bar{\sigma}_{m n}^{x}\right)
$$

with $E(\theta)$ replaced by $\widetilde{E}(\theta)$ in $(5.6 .12)_{\mathscr{R}_{2}}$. 
Remark. The correlation function $(5.7 .14)^{(*)}$ coincides with that of Ising spins on the dual lattice. See [14] for the detailed discussions on this point. We only note the values of parameters (5.7.26) for i) the triangular and ii) the generalized square lattices.

i) The triangular lattice.

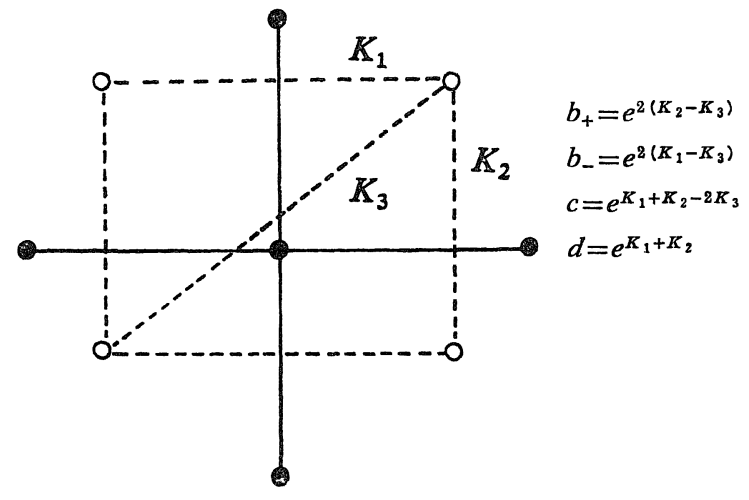

ii) The generalized square lattice.

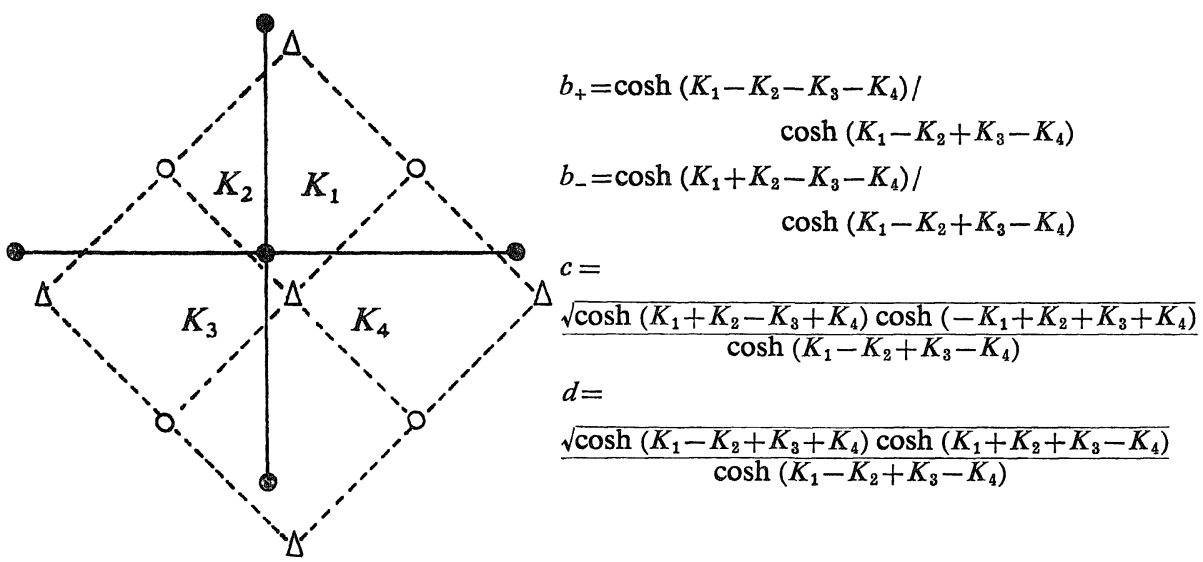

\section{References}

[1] Sato, M., Miwa, T. and Jimbo, M., Publ. RIMS, Kyoto Univ., 14 (1978), 223-267.

[2] — Proc. Japan Acad., 53A (1977), 6-10.

[3] — Publ. RIMS, Kyoto Univ., 15 (1979), 871-972. See also Publ. RIMS, Kyoto Univ., 15 (1979), 201-278, ibid., 577-629.

(*) Infinite series expressions are obtained by substituting the above results into (5. 6. 14)(5. 6. 17). 
[4] - Field theory of the two-dimensional Ising model in the scaling limit, RIMS preprint, 207 (1976).

[ 5 ] Onsager, L., Phys. Rev., 65 (1944), 117-149.

[6] McCoy, B. M. and Wu, T. T., The two-dimensional Ising model, Harvard University Press, 1973.

[7] Schultz, T. D., Mattis, D. C. and Lieb, E. H., Rev. Mod. Phys., 36 (1964), 856-871.

[ 8] Kaufman, B., Phys. Rer., 76 (1949), 1232-1243.

[9] McCoy, B. M., Tracy, C. A. and Wu, T. T., Phys. Rev. Lett., 38 (1977), 793-796.

[10] Bariev, R. Z., Phys. Lett., 64A (1977), 169-171.

[11] Abraham, D. B., Commun. Math. Phys., 59 (1978), 17-34.

[12] See e.g. the book of Domb and Green, Phase transitions and critical phenomena 1, Academic Press, 1972.

[13] Vaidya, H. G. and Tracy, C. A., Physica, 92A (1978), 1-41.

[14] Green, H. S. and Hurst, C. A., Order-disorder phenomena, Interscience, 1964.

[15] Fan, C. and Wu, F. Y., Phys. Rev., 179 (1969), 560-570.

[16] Felderhof, B. U., Physica, 65 (1973), 421-451, 60 (1973), 279-297, 509-526.

[17] Szegö, G., Commun. Seminar. Math. Univ. Lund, suppl. dédié à Marcel Riesz, 228 (1952). An account on this topic is also given in the book [6].

[18] Vaidya, H. G., Phys. Lett., 57A (1976), 1-4.

Note added in proof: The authors are indebted to Professor C.A. Tracy for drawing their attention to the following article: C.A. Tracy and B.M. McCoy, Phys. Rev. Lett., 31 (1973), 1500-1504, which should be added to the references of this series on Holonomic Quantum Fields. After completion of the manuscript they also learned that the path formulation of order-disorder variables in Sections 5.4 and 5.7 originates in the work of Kadanoff and Ceva (Phys. Rev., B3 (1971), 3918-3939). 